\title{
CONICAL VECTORS IN INDUCED MODULES
}

\author{
BY
}

\section{J. LEPOWSKY $\left.{ }^{1}\right)$}

ABST RACT. Let $g$ be a real semisimple Lie algebra with Iwasawa decomposition $g=t \oplus a \oplus n$, and let $m$ be the centralizer of $a$ in $t$. A conical vector in a $g$-module is defined to be a nonzero $m \oplus n$-invariant vector. The g-modules which are algebraically induced from one-dimensional ( $m \oplus a \oplus n)$ modules on which the action of $m$ is trivial have "canonical generators" which are conical vectors. In this paper, all the conical vectors in these 9 -modules are found, in the special case $\operatorname{dim} a=1$. The conical vectors have interesting expressions as polynomials in two variables which factor into linear or quadratic factors. Because it is too difficult to determine the conical vectors by direct computation, metamathematical "transfer principles" are proved, to transfer theorems about conical vectors from one Lie algebra to another; this reduces the problem to a special case which can be solved. The whole study is carried out for semisimple symmetric Lie algebras with splitting Cartan subspaces, over arbitrary fields of characteristic zero. An exposition of the Kostant-Mostow double transitivity theorem is included.

1. Introduction. The theory of Verma modules, as developed by D.-N. Verma [10(a), (b)] and by I. N. Bernštein, I. M. Gel'fand and S. I. Gel'fand $[1(a),(b)]$, is becoming increasingly important. Let $g$ be a complex semisimple Lie algebra and $\mathfrak{b}$ a Borel subalgebra of $g$. The associated Verma modules are the $g$-modules induced, in the algebraic sense, by the one-dimensional b-modules (see [2, Chapter 7]). As we shall see in this introduction, a corresponding theory of $g$-modules induced from more general parabolic subalgebras of $g$ should also be developed, and the purpose of this paper is to begin such a study.

Here is our main reason for interest in this problem: Let $G=K A N$ be an Iwasawa decomposition of a real semisimple Lie group with finite center, and

Received by the editors March 14, 1974 and, in revised form, July 15, 1974.

AMS (MOS) subject classifications (1970). Primary 17B 10; Secondary 16A64, 17B20, 17B35, 22E45.

Key words and phrases. Conical vectors, highest weight vectors, induced modules, Verma modules, real semi simple Lie algebras, real rank one, semisimple symmetric Lie algebras, splitting Cartan subspaces, restrícted roots, restricted weight vectors, restricted Weyl group, universal enveloping algebra, double transitivity theorem, polynomial invariants.

(1) Partially supported by NSF GP-33893. 
$g=\mathfrak{E} \oplus a \oplus n$ the corresponding decomposition of the complexified Lie algebra of $G$. Let $M$ be the centralizer of $A$ in $K$, and $m$ its complexified Lie algebra. The infinitesimal nonunitary principal series of $G$ is the family of $g$-modules obtained by taking the $K$-finite subspaces of the nonunitary principal series representations-those Hilbert space representations of $G$ induced from the finite-dimensional irreducible representations of $M A N$ (see for example [7(a)]). This family of $\mathrm{g}$-modules is of great importance because every irreducible $g$-module which splits into a direct sum of finite-dimensional irreducible ${ }^{k}$-modules exponentiating to $K$-modules is a subquotient of an infinitesimal nonunitary principal series module (see [4], [7(a)], [9] and [2, Chapter 9]). But roughly speaking, the infinitesimal nonunitary principal series modules may be identified with certain "large" subspaces of the contragredient $g$-modules to $g$-modules algebraically induced by finite-dimensional irreducible modules of the parabolic subalgebra $m \oplus a \oplus \mathfrak{n}$ of $g$ (cf. $[2, \$ \$ 9.3 .1$, 9.7.101). Other important families of induced representations of $G$ are similarly related to $g$-modules algebraically induced from parabolic subalgebras of $g$.

In a sense, the algebraically induced modules may be thought of as modules of distributions supported at the identity element of $G$, and their dualsalgebraically "produced" modules-as modules of formal power series at the identity element of $G$. The $K$-finite elements of the produced modules (the $K$ finite formal power series) then correspond to analytic functions on $G$ which are also the $K$-finite elements of the Hilbert space induced representations.

The Verma modules that can be embedded in a given Verma module are completely known ([10l and [1(a)]; see also [2, Théorème 7.6.23]). Suppose one could correspondingly determine the $g$-module maps between pairs of $g$ modules algebraically induced from $m \oplus a \oplus n$. Looking at the dual maps between the $K$-finite subspaces of the contragredient modules, one would have intertwining operators between nonunitary principal series $G$-modules, and these intertwining operators, which might be Kunze-Stein integral operators, would now be given by differential formulas. Furthermore, since an algebraically induced module is generated by a "highest weight vector" ( $n$-invariant vector), the $g$-maps from one of the algebraically induced modules to another are closely related to the highest weight vectors in the target module. These give rise to highest weight vectors in the dual of the $K$-finite subspace of the Hilbert space induced $G$-module, and therefore are intimately connected with S. Helgason's conical distributions $[5(a),(b)] .\left({ }^{2}\right)$ The submodule structure of

(2) See al so M. Hu's thesis [12], whose results on conical distributions are related to our results on conical vectors. 
the algebraically induced $g$-modules must also shed light on the subquotient structure of the nonunitary principal series modules (see M. Duflo [3] and [2, $\$ 9.6]$ for the case of complex $G$, using Verma modules), but examples show that the relation will be subtle. For instance, irreducibility of the algebraically induced module is not equivalent to irreducibility of the related contragredient nonunitary principal series module. On the other hand, the subquotient structure of the nonunitary principal series is notoriously complicated, but the structure of the algebraically induced modules already appears to be more regular and perhaps more fundamental. For example, the inclusion relations among the Verma submodules of certain Verma modules recover the inclusion relations among the closures of the Bruhat cells for complex semisimple Lie groups (see [10]), and it is likely that this situation will generalize to real semisimple Lie groups, using the modules algebraically induced from $\mathfrak{m} \oplus a \oplus \mathfrak{n}$.

Now that we want to find the highest weight vectors in a given $g$-module $X$ algebraically induced from a finite-dimensional irreducible $(m \oplus a \oplus n)$ module, how do we do it? The following seemed at first like a good starting point: Let $\mathfrak{l}$ be a Cartan subalgebra of $m$, so that $\mathfrak{G}=\mathfrak{l} \oplus a$ is a Cartan subalgebra of $g$. Let $\mathfrak{b}$ be a Borel subalgebra of $g$ containing $\mathfrak{G}$ and $n$. Then it is easy to see that $X$ is a $g$-module quotient of a certain Verma module $V$ induced from $b$ (cf. [2, Lemma 9.3.2]). Hence one can try to use the well-developed theory of highest weight vectors in Verma modules to study highest weight vectors in $X$. Unfortunately, however, highest weight vectors in $V$ can vanish when one passes to the quotient $X$, even in simple examples. Moreover, it turns out that there are, in general, highest weight vectors in $X$ which do not come from highest weight vectors in $V$. This subtlety, which made the problem much more difficult than we expected it to be, forced us to work in a relatively special case and to develop new tools to handle even this case.

Now we shall describe our main results, and then we shall say what is interesting about our methods.

By analogy with Helgason's conical distributions, we call a nonzero vector in a $g$-module (or more generally, in an $m \oplus n$-module) conical if it is $m \oplus n$-invariant. The space of conical vectors, together $w$ ith 0 , is called the conical space of the module. Let $\mathcal{G}$ be the universal enveloping algebra of $g$ and $\mathcal{P} \subset \mathcal{Q}$ the universal enveloping algebra of $m \oplus a \oplus n$. Define $\rho \in a^{*}$ (* denotes dual) by the condition $\rho(a)=1 / 2 \operatorname{tr}(\operatorname{ad} a \mid n)$ for all $a \in a$, so that $\rho$ is half the sum of the positive restricted roots with multiplicities counted. For all $\nu \in a^{*}$, the linear functional on $m \oplus a \oplus n$ which is zero on $m \oplus n$ and 
$\nu-\rho$ on $a$ defines a one-dimensional representation of $m \oplus a \oplus n$. Regarding $\mathbf{C}$ as the associated one-dimensional $\mathscr{P}_{\text {-module, and }} \mathcal{S}$ as a right $\mathcal{P}_{\text {-mod- }}$ ule by right multiplication, we can form the $\mathcal{G}_{\text {-module }} X^{\nu}=\varrho \otimes_{\varphi} \mathbf{C}$. This is a "twisted induced module" in the sense of $[2, \$ 5.2]$. The vector $x_{0}=1 \otimes$ $1 \in X^{\nu}$ is a conical vector which generates $X^{\nu}$, and is called the canonical generator of $X^{\nu}$. Let $n-C g$ be the sum of the negative restricted root spaces of $g$ with respect to $a$, and $\pi-\subset \varrho$ its universal enveloping algebra. Then $x^{\nu}=\pi-\cdot x_{0}$.

We are aiming for a description of the conical vectors in $X^{\nu}$ in case $G$ has real rank 1 , i.e., $\operatorname{dim} a=1$. Assume this, and let $\alpha \in a^{*}$ be the unique simple restricted root. Then $n^{-}$is the direct sum of the restricted root spaces $g^{-\alpha}$ and $g^{-2 \alpha}$; here $g^{-2 a}$ may be zero. There are natural $M$-invariant nonsingular symmetric bilinear forms on $g^{-a}$ and $g^{-2 a}$. Let $q_{-a} \in \Re^{-}$and $q_{-2 a}$ $\epsilon \gamma^{-}$be the sums of the squares of orthonormal bases of $g^{-a}$ and $g^{-2 a}$, respectively, so that $q_{-\alpha}$ and $q_{-2 a}$ are quadratic M-invariant elements of $\pi^{-}$, and $q_{-2 a}=0$ if $g^{-2 a}=0$. Let $\left(\mathcal{H}^{-}\right)^{M}$ be the algebra of all $M$-invariants in $\gamma^{-}$. Then $\left(\pi^{-}\right)^{M}$ is a polynomial algebra on either one or two generators, depending on whether $g^{-2 a}=0$ or $g^{-2 a} \neq 0$ (see $\$ 5$ ), and in the difficult case when $\operatorname{dim} g^{-2 a}>1$, the two generators are $q_{-} a$ and $q_{-2 \alpha}$; this follows from the Kostant-Mostow double transitivity theorem (see $\$ 4$ ) on M-orbits in $n^{-}$ (or more precisely, $M$-orbits in the intersection of $n^{-}$with the real Lie algebra of $G$ ). With this as background, we now state our main results (see $\$ 10$ ):

Theorem 1.1. Assume $\operatorname{dim} a=1$ and let $\nu \in a^{*}$. Then the conical space of $X^{\nu}$ is either one-or two-dimensional, according to whether $\nu$ is a positive integral multiple of $a\left(\right.$ of $1 / 2 \alpha$ if $\operatorname{dim} g^{a}=1$ ) or not. If $\nu$ is not of this form, then the conical space of $X^{\nu}$ is spanned by the canonical generator $x_{0}$ of $X^{\nu}$. Suppose $\nu=l a, l$ a positive integer. (If $\operatorname{dim} g^{a}=1$, take instead $\nu=$ $1 / 2$ la.) Then $q_{-a}$ and $q_{-2 a}$ can be suitably renormalized (independently of $i$ ) so that the following is true: Suppose $\operatorname{dim} g^{a}>1$. Define $\zeta_{l} \in \Re^{-}$by the formula

$$
\zeta_{l}=\left\{\begin{array}{cl}
\prod_{j=1 ; j \text { odd }}^{l}\left(q_{-a}^{2}+j^{2} q_{-2 \alpha}\right), & \text { l even, } \\
q_{-a} \prod_{j=2 ; j \text { even }}^{l-1}\left(q_{-a}^{2}+j^{2} q_{-2 \alpha}\right), & l \text { odd. }
\end{array}\right.
$$

If $\operatorname{dim} g^{\alpha}=1$, define $\zeta_{l}=f^{l} \in \pi^{-}$, where $f$ is a nonzero element of $g^{-a}$. Then the conical space of $X^{\nu}$ has basis $\left\{x_{0}, \zeta_{l} \cdot x_{0}\right\}$. Moreover, the g-sub. module of $X^{\nu}$ generated by $\zeta_{l} \cdot x_{0}$ is isomorphic to $X^{-\nu}$. 
Theorem 1.2. Let $\mu, \nu \in a^{*}$. Then $\operatorname{dim} \operatorname{Hom}_{g}\left(X^{\mu}, X^{\nu}\right) \leq 1$. Moreover, $\operatorname{dim} \operatorname{Hom}_{g}\left(X^{\mu}, X^{\nu}\right)=1$ if and only if either $\mu=\nu$, or else $\mu=-\nu$ and $\nu$ is a nonnegative integral multiple of $a\left(\right.$ of $1 / 2 a$ if $\left.\operatorname{dim} g^{a}=1\right)$. This is exactly the case in which $X^{\mu}$ is isomorphic to a g-submodule of $X^{\nu}$.

(The annoying exceptional case $\operatorname{dim} g^{a}=1$ in these two theorems is essentially the case $G=S L(2, \mathbf{R})$, and is trivial.)

Considering how rare it is for a polynomial in two variables to factor into linear or quadratic factors, the factored form of the $\zeta_{l}$ in Theorem 1.1 seems remarkable. We shall say more about this below.

It turns out that Theorem 1.2 follows easily from Theorem 1.1, so we shall explain what is involved in proving Theorem 1.1. First, it is easy to see that the space of $m$-invariants in $X^{\nu}$ is the space $\left(\eta^{-}\right)^{m} \cdot x_{0}$ (here $\left(r^{-}\right)^{m}$ is the space of $m$-invariants in $\Re^{-}$and equals $\left.\left(\gamma^{-}\right)^{M}\right)$. From the above, $(\pi-)^{m}$ is a polynomial algebra in one or two generators. If $g^{2 a}=0$, we have one generator, and Theorem 1.1 is not terribly hard in this case (see $\$ 6$ ). Suppose now that $\operatorname{dim} g^{2 \alpha}>1$, so that $\left(\pi^{-}\right)^{\mathrm{m}}$ is the polynomial algebra $\mathrm{C}\left[q_{-a}, q_{-2 a}\right]$. The whole problem is to determine those polynomials $p$ in two variables such that $p\left(q_{-a}, q_{-2 a}\right) \cdot x_{0}$ is $n$-invariant. Clearly, this involves computing commutators of elements of $n$ with $q_{-a}$ and $q_{-2 a}$, and also commutators of these commutators with $q_{-a}$ and $q_{-2 a}$. We were able to compute the necessary commutators (see $\$ \$ 6,7$ ), but the resulting condition on the polynomial $p$ is immensely complicated, and it is not feasible to analyze it directly (see the last remark in $\$ 8$ ).

However, when attempting to unravel this condition on $p$ for some special $G$ 's, we noticed that the computations, even though we could not do them for any one $G$, did not seem to depend on $G$. The key was then to prove $a$ priori that the conical vectors would look the same for any one $G$ (for which $\operatorname{dim} g^{2 a}>1$ ) as for any other such $G$, and then to use possibly special methods to solve the problem for one "small" G. Specifically, we first proved what we call the "fundamental commutation relation in $\pi^{-"}$ : There is a nonzero constant $c \in \mathbf{C}$ such that $\left[\left[f, q_{-a}\right], q_{-a}\right]=c f q_{-2 a}$ for all $f \in g^{-a}$ (see Theorem 7.4): This is called "fundamental" because of the next result: If $f$ is chosen more carefully, then this relation and a trivial one $\left(\left[f, q_{-2 a}\right]=0\right)$ generate all relations which are linear in $f$ in the associative subalgebra of $r^{-}$generated by $f, q_{-a}$ and $q_{-2 a}$ (see Theorem 8.1). This in turn implies the following metamathematical "transfer principle for $\gamma^{-}$": If $a_{1}, \ldots, a_{r}$, $b_{1}, \ldots, b_{r}$ are complex polynomials in two variables, then the truth of any assertion of the form " $\Sigma_{i=1}^{r} a_{i}\left(q_{-a}, q_{-2 a}\right) f b_{i}\left(q_{-a}, q_{-2 a}\right)=0$ in $\pi-"$ is in- 
dependent of $G$ (see Theorem 8.4). But the condition that $p\left(q_{-a}, q_{-2 a}\right) \cdot x_{0}$ be conical in $X^{\nu}$ can be expressed in this form (see Lemma 8.5), where the $a_{i}$ and $b_{i}$ depend only on $p$ and the complex number $c$ such that $\nu=c a$. Thus we could prove the "transfer principle for conical vectors", another metatheorem which says that if $p\left(q_{-a}, q_{-2 a}\right) \cdot x_{0}$ is conical in $X^{c a}$ for some $G$ with $\operatorname{dim} g^{2 a}>1$, then the same is true for any such $G$ (see Theorem 8.6). Furthermore, the above metatheorems have analogues for the case $\operatorname{dim} g^{2 a}=1$, enabling us even to transfer theorems about conical vectors from any one $G$ with $\operatorname{dim} g^{2 a}=1$ to any $G$ with either $\operatorname{dim} g^{2 a}=1$ or $\operatorname{dim} g^{2 a}>1$ (see Theorems 8.4 and 8.6).

The conical vectors still had to be computed for some special $G$ with $\operatorname{dim} g^{2 a} \geq 1$. The only cases which we were able to do directly, aided by a crucial observation of $\mathrm{L}$. Corwin, were the cases $G=S U(n, 1)$-essentially all the $G^{\prime}$ s such that $\operatorname{dim} g^{2 a}=1$. In these cases, $(\pi-)^{m}$ is the polynomial algebra in $q_{-a}$ and $r_{-2 a}$, where $r_{-2 \alpha}$ is a nonzero element of the one-dimensional space $g^{-2 a}$. We reformulated the condition that $p\left(q_{-a}, r_{-2 a}\right) \cdot x_{0}$ be conical in $X^{\nu}$ (where $p$ is a complex polynomial in two variables) in terms of a complicated system of linear equations whose unknowns were essentially the coefficients of $p$. These equations implied uniqueness of the conical vectors, but it was not clear that the equations had a consistent solution (and hence it was not clear that the conical vectors in Theorem 1.1 existed) until Corwin noticed that a solution vector could be constructed from the coefficients of a certain polynomial which factored into certain linear factors. This meant that if $p$ were this polynomial, then $p\left(q_{-a}, r_{-2 a}\right) \cdot x_{0}$ would be conical. This was enough to prove Theorem 1.1 for these $G$ 's. To place the case $\operatorname{dim} 9^{2 a}=1$ in perspective, we further note the following: In this case, $r_{-2 a}^{2}$ $=q_{-2 a}$ in $\pi^{-}$, and therefore the factors $q_{-a}^{2}+j^{2} q_{-2 a}$ in Theorem 1.1 themselves factor into linear factors: $\left(q_{-\alpha}+(-1)^{1 / 2} j r_{-2 \alpha}\right)\left(q_{-a}-(-1)^{1 / 2} j r_{-2 \alpha}\right)$. It was this which made it feasible to carry out the necessary computations (see the Remark following Lemma 9.1).

Actually, in writing up the special case in $\$ 9$, we dealt only with $G=$ $S U(2,1)$, and following a suggestion of $\mathrm{N}$. Wallach, we used the the ory of Verma modules to prove the uniqueness of the conical vectors. (For $G=$ $S U(2,1)$, the $g$-module induced from $m \oplus a \oplus n$ is actually a Verma module, not just a quotient.) Thus the original approach, using the complicated system of linear equations, is not carried out in this paper.

The above results are stated for $G$ of real rank 1, but they imply a result for arbitrary real rank, included in Theorems 10.1 and 10.2. 
There is another direction in which Theorems 1.1 and 1.2 are extended in this paper-to arbitrary fields of characteristic zero. In fact, throughout this paper, we work with semisimple symmetric Lie algebras with splitting Cartan subspaces, over fields of characteristic zero (see [2] and [7(b)] for background on these). This accounts for most of the length of $\$ \$ 2-4$, in which we wanted to give a self-contained elementary treatment of the Kostant-Mostow double transitivity theorem and its consequences for algebras of polynomial invariants, valid over general fields of characteristic zero, without using any theory of Lie or algebraic groups. Instead of group orbits, we use "infinitesimal transitivity and double transitivity" conditions. We essentially give Wallach's modified version of Kostant's proof of the double transitivity theorem. See $\$ \S 3$ and 4 for a more detailed discussion of this theorem and its consequences.

Incidentally, it is not surprising that theorems about real semisimple Lie algebras, Cartan decompositions and Iwasawa decompositions should also hold for more general semisimple symmetric Lie algebras, since joint work with G. McCollum has shown that assertions about such structures whose truth is preserved under field extension and restriction are true for any one field of characteristic zero if and only if they are true for any other; see [8(e)]. This gives a generalization of $\mathrm{H}$. Weyl's "unitary trick", which enables one to transfer theorems from compact semisimple Lie algebras to semisimple Lie algebras over arbitrary fields of characteristic zero.

After the work for this paper was completed, we found a simpler proof of the uniqueness of the conical vectors, avoiding the use of the double transitivity theorem; see [8(d)]. (But the existence and explicit form of the conical vectors still require the fundamental commutation relation and transfer principles.) This proof uses an observation of Kostant on the limitations imposed on conical vectors by the action of the center of $\mathcal{G}$. The proof also uses an a priori argument that the first assertion of Theorem 1.2 holds-that $\operatorname{dim} \operatorname{Hom}_{g}\left(X^{\mu}, X^{\nu}\right) \leq 1$. In fact, we have generalized this last inequality to all parabolic subalgebras (see [8(c)]) by extending the method that Verma originally used (see [2, Théorème 7.6.6]) to prove the corresponding fact about Verma modules.

We remarked above that a $g$-module $X$ induced from a finite-dimensional irreducible $(m \oplus a \oplus n)$-module is a quotient of a certain Verma module $V$, but that one cannot very well use $V$ to determine the highest weight vectors in $X$. On the other hand, since Theorems 1.1 and 1.2 are true, we can use them as a tool in investigating the composition series of the Verma module $V$. Interesting things happen: First, recall that in [1(a)], Bernštein, Gel'fand and 
Gel'fand found an example of a Verma module for $2 L(4, C)$ having two strange properties: It contains a proper submodule not generated by Verma submodules, and its composition series contains a certain irreducible subquotient with multiplicity two. But it now turns out that if one regards $2 L(4, C)$ as the complexification of $\mathcal{Q} u(3,1)$, then one can explain all of this pathology by means of the existence of a certain conical vector in $X$ which does not come from a highest weight vector in $V$. In effect, Bernstein, Gel'fand and Gel'fand were actually dealing with the case $l=1, \zeta_{l}=q_{-\alpha}$ in Theorem 1.1. Moreover, using Theorem 1.1, we can generate whole families of examples of the same two "strange" phenomena for many Lie algebras. Thus a "bad" phenomenon for Verma modules becomes "good" when one interprets the situation using a larger parabolic subalgebra than a Borel subalgebra. This further emphasizes the importance of studying modules induced from general parabolic subalgebras.

Along the same lines, we comment that the results of [1] and [10] do not, in general, give explicit expressions for the highest weight vectors in a Verma module, or equivalently, explicit formulas for the embedding of one Verma module into another; they usually give only the existence of the vectors or the embeddings. But we can use the polynomials $\zeta_{l}$ in Theorem 1.1 to give explicit expressions for certain of these highest weight vectors or embeddings which have not yet been described explicitly.

We would like to thank G. D. Mostow for informing us about his approach to the double transitivity theorem.

Notations. We shall write $\mathbf{Z}_{+}$for the set of nonnegative integers and $\mathbf{Q}$ for the field of rational numbers. Throughout this paper, $k$ is a field of characteristic zero. The dual of a vector space $V$ over $k$ is denoted $V^{*}$. The symmetric algebra of $V$ is written $S(V)$, and for all $r \in Z_{+}$, the $r$ th symmetric power is denoted $S^{r}(V)$, so that

$$
S(V)=\coprod_{r \in \mathrm{Z}_{+}} S^{r}(V)
$$

$S\left(V^{*}\right)$ is naturally isomorphic to the algebra of polynomial functions on $V$ (i.e., the algebra of sums of products of linear functions on $V$ ), and we shall often identify these two algebras. Let $g$ be a Lie algebra over $k$, and let $V$ be a $g$-module. Then $g$ may be canonically embedded in the universal envel-

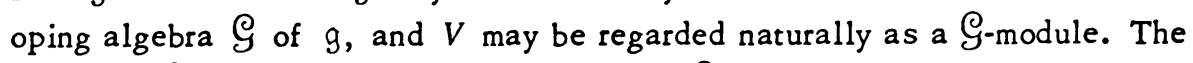
action of $\mathcal{G}$ on $V$ will be denoted $x \cdot v(x \in \mathcal{G}, v \in V)$. If $\delta$ and $T$ are subsets of $g$ and $V$, respectively, let $T^{8}$ be the set of $z$-invariants in $T$, i.e., $\{t \in T \mid s \cdot t=0$ for all $s \in B\}$. Regard $\mathcal{G}$ and $S(g)$ as $g$-modules by the natural extensions by derivations of the adjoint action of $g$ on itself. Then for 
$x \in g$ and $y \in \mathcal{G}, x \cdot y=[x, y]$, where we use $[\cdot, \cdot]$ to denote the commutator in associative algebras, as well as the bracket in Lie algebras. In particular, if $B \subset g$ and $T \subset \mathcal{G}$, then $T^{B}$ is the ordinary centralizer of $B$ in $T$. Note that for all $x \in g, y \in \oint$ and $v \in V$, we have $x \cdot(y \cdot v)=[x, y] \cdot v+y \cdot$ $(x \cdot v)$. Regard $V^{*}$ as the $g$-module contragredient to the g-module $V$.

2. The setting. Here we shall summarize the necessary preliminaries and fix notation to be used throughout most of this paper.

Let $(g, \theta)$ be a semisimple symmetric Lie algebra over $k$, i.e., $g$ is a semisimple Lie algebra over $k$ and $\theta$ is an automorphism of $g$ such that $\theta^{2}=1$. (See [2] and [7(b)] for background information on semisimple symmetric Lie algebras.) Denote by $\mathcal{E}$ and $\{$ the +1 and -1 eigenspaces for $\theta$,

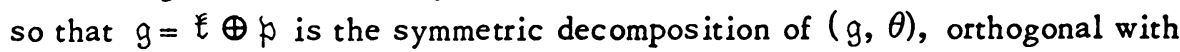
respect to the Killing form of $\mathrm{g}$. Assume that there is a splitting Cartan subspace $a$ of $p$. That is, $a$ is a maximal abelian subspace of $p$ whose adjoint action on $\mathrm{g}$ can be simultaneously diagonalized.

Let $m$ be the centralizer of $a$ in $E$, and for all $k$-linear functionals $\phi: a \rightarrow k$, define

$$
g^{\phi}=\{x \in g \mid[a, x]=\phi(a) x \text { for all } a \in a\} .
$$

Then $g^{0}=\mathfrak{m} \oplus a$. Let

$$
\Sigma=\left\{\phi \in a^{*} \mid \phi \neq 0 \text { and } g^{\phi} \neq 0\right\},
$$

the set of restricted roots of $g$ with respect to $a$. Then

$$
g=g^{0} \oplus \coprod_{\phi \in \Sigma} g^{\phi}=m \oplus a \oplus \coprod_{\phi \in \Sigma} g^{\phi} .
$$

Moreover, $\left[g^{\phi}, g^{\psi}\right] \subset g^{\phi \oplus \psi}$ and $\theta g^{\phi}=g^{-\phi}$ for all $\phi, \psi \in a^{*}$.

Let $B$ be the Xilling form of g. Then $B$ is nonsingular on a (see [7(b)]), so that $B$ induces naturally a nonsingular symmetric $k$-bilinear form $(\cdot, \cdot)$ on $a^{*}$, as well as a natural isometry between $a$ and $a^{*}$. Let $a_{Q^{*}}^{*}$ denote the rational span of $\Sigma$ in $a^{*}$. Then $a^{*}$ is naturally isomorphic to $a_{Q}^{*} \otimes_{\mathbf{Q}} k$, and the form $(\cdot, \cdot)$ is rational-valued and positive definite on the rational space $a_{Q}^{*}($ see $[7(b)])$. In particular, $(\phi, \phi) \neq 0$ for all $\phi \in \Sigma$.

For all $\phi \in \Sigma$, let $s_{\phi}$ denote the orthogonal reflection of $a^{*}$ through the hyperplane perpendicular to $\phi$, and let $W$ be the group of isometries of $a^{*}$ generated by the $s_{\phi}(\phi \in \Sigma) . W$ is called the restricted Weyl group of $g$ with respect to $a . \Sigma$ spans $a^{*}$ and forms a (not necessarily reduced) system of roots in $a^{*}$ with Weyl group $W($ see [7(b), \$2]).

Let $\Sigma_{+}$be a positive system in $\Sigma$, and define 


$$
n=\prod_{\phi \in \Sigma_{+}} g^{\phi} \text { and } n^{-}=\coprod_{\phi \in \Sigma_{+}} g^{-\phi} \text {. }
$$

Then $n$ and $n^{-}$are nilpotent subalgebras of $g$, and we have the decomposition $g=n^{-} \oplus m \oplus a \oplus n$.

Define the bilinear form $B_{\theta}$ on $g$ by the condition $B_{\theta}(x, y)=-B(x, \theta y)$ $(x, y \in g)$. Then $B_{\theta}$ is a nonsingular symmetric form, and the decomposition $g=m \oplus a \oplus \amalg_{\phi \in \Sigma} g^{\phi}$ is a $B_{\theta}$-orthogonal decomposition (see [7(b), Lemma 3.2]). Hence $B_{\theta}$ is nonsingular on each $g^{\phi}(\phi \in \Sigma)$ on $m$ and on a. More-

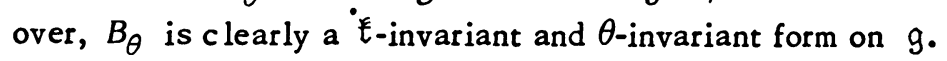

For all $\phi \in \Sigma$, let $x_{\phi} \in a$ denote the image of $\phi$ under the canonical isometry from $a^{*}$ to $a$, so that $B\left(x_{\phi}, a\right)=\phi(a)$ for all $a \in a$, and $B\left(x_{\phi}, x_{\psi}\right)$ $=(\phi, \psi)$ for all $\phi, \psi \in \Sigma$. Then for all $e \in g^{\phi},[e, \theta e] \in a$, and in fact

$$
[e, \theta e]=B(e, \theta e) x_{\phi}=-B_{\theta}(e, e) x_{\phi}
$$

[7(b), Lemma 3.3]. Since $(\phi, \phi) \neq 0$, we can define $h_{\phi}=2 x_{\phi} /(\phi, \phi) \in a$. Then $\phi\left(h_{\phi}\right)=2$.

Suppose now that $k$ is algebraically closed, so that every element in $k$ has a square root. Since $B_{\theta}$ is a symmetric nonsingular form on $g^{\phi}, g^{\phi}$ contains a nonisotropic vector $e_{0}$ with respect to the form $B_{\theta}$ (i.e., $B_{\theta}\left(e_{0}, e_{0}\right)$ $\neq 0)$. Set

$$
e_{\phi}=\left(2 /(\phi, \phi) B_{\theta}\left(e_{0}, e_{0}\right)\right)^{1 / 2} e_{0}
$$

and $f_{\phi}=-\theta e_{\phi}$. Then $B_{\theta}\left(e_{\phi}, e_{\phi}\right)=2 /(\phi, \phi)$, and so $\left[h_{\phi}, e_{\phi}\right]=2 e_{\phi},\left[h_{\phi}, f_{\phi}\right]$ $=-2 f_{\phi}$ and $\left[e_{\phi}, f_{\phi}\right]=h_{\phi}$. Hence $\left\{h_{\phi}, e_{\phi}, f_{\phi}\right\}$ spans a three-dimensionalsimple subalgebra $u_{\phi}$ of $g$.

Now drop the algebraic closure assumption on $k$. Let $\mathcal{G}$ be the universal enveloping algebra of $g$, and let $\pi, \mathbb{Q}, \pi$ and $\pi$ - denote the universal enveloping algebras of $m, a, n$ and $n^{-}$, respectively, regarded as canonically embedded in $\mathrm{g}$. Then the multiplication map in $\mathcal{G}$ induces a linear isomorphism

$$
\mathcal{G} \simeq-\otimes \pi \otimes Q \otimes r
$$

Let $\nu \in a^{*}$. Then the linear form on the subalgebra $m \oplus a \oplus n$ of $g$ which is $\nu$ on $a$ and zero on $m \oplus n$ vanishes on the commutator subalgebra of $m \oplus a \oplus n$, and thus corresponds to a one-dimensional representation $\pi$ of $m \oplus a \oplus n$ and hence of its universal enveloping algebra M( $m$. Let $V^{2}$ be the $g$-module induced by the $(m \oplus a \oplus n)$-module defined by $\pi$ (see [2, $\$ 5.1])$. That is, 


$$
V^{\nu}=\mathcal{G} \otimes_{\operatorname{mar}} k \text {, }
$$

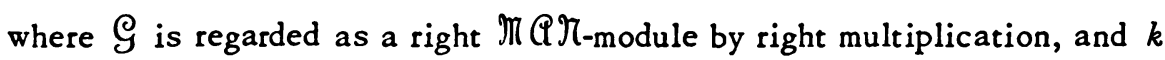

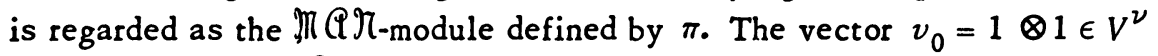
generates $V^{\nu}$ as a $\oint$-module, and is called the canonical generator of $V^{\nu}$. It is clear that the map $\omega: r^{-} \rightarrow V^{\nu}$ given by $x \mapsto x \cdot v_{0}$ is a linear isomorphism.

Let $V$ be a g-module, $v \in V$ a nonzero vector and $\lambda \in a^{*}$. Then $v$ is called a restricted weight vector and $\lambda$ a restricted weight for $V$ if $x \cdot v=$ $\lambda(x) v$ for all $x \in a$. For all $\lambda \in a^{*}$, the subspace of $V$ consisting of 0 and the restricted weight vectors for $\lambda$ is called the restricted weight space for $\lambda$; it is nonzero if and only if $\lambda$ is a restricted weight for $V$.

The following definitions are central to this paper: Let $V$ be a $g$-module, and let $v \in V$ be nonzero. Then $v$ is a conical vector for $V$ if $v \in V^{\mathrm{m} \oplus \mathfrak{n}}$, i.e., if $(m \oplus n) \cdot v=0$. The subspace $V^{\mathfrak{m} \oplus \mathfrak{n}}$ consisting of 0 and the conical vectors is called the conical space of $V$.

Now let $\nu \in a^{*}$ and let $v_{0}$ be the canonical generator of the induced module $V^{\nu}$. Then $v_{0}$ is clearly a conical restricted weight vector in $V^{\nu}$ with restricted weight $\nu$. It is also clear that the conical space of $V^{\nu}$ is a-invariant and hence is the direct sum of its intersections with the restricted weight spaces of $V^{\nu}$.

The standard universal property of the induced module $V^{\nu}$ (see $[2, \$ 5.1]$ ) say that if $U$ is a $g$-module and $u \in U$ is a conical restricted weight vector with restricted weight $\nu$, then there is a unique $g$-module homomorphism $f: V^{\nu} \rightarrow U$ such that $f\left(v_{0}\right)=u$. If $u$ generates $U$, then $f$ is surjective. If $U=V^{\mu}$ for some $\mu \in a^{*}$, then $f$ is injective; this follows from the fact that $\pi$ - has no zero divisors. Let $Z \subset V^{\mu}$ be the intersection of the conical space and the restricted weight space for $\nu$. Then we have a natural linear isomorphism

$$
\operatorname{Hom}_{9}\left(V^{\nu}, V^{\mu}\right) \rightarrow Z, \quad f \mapsto f\left(v_{0}\right) .
$$

Let $\nu$ and $v_{0}$ be as above. Since $v_{0} \in\left(V^{\nu}\right)^{\mathrm{m}}$, the linear isomorphism $\omega: \pi-\rightarrow V^{\nu}$ (see above) is also an m-module isomorphism, where $r^{-}$is regarded as an $m$-submodule of $\mathcal{G}$ under the adjoint action. In particular, $\left(V^{\nu}\right)^{\mathrm{m}}=\left(\tau^{-}\right)^{\mathrm{m}} \cdot v_{0}$, and in fact $\omega$ restricts to a linear isomorphism

$$
\omega:\left(r^{-}\right)^{m} \rightarrow\left(V^{\nu}\right)^{m}, \quad x \mapsto x \cdot v_{0} .
$$

Define $\rho \in a^{*}$ by the formula

$$
\text { . } \rho(a)=1 / 2 \operatorname{tr}(\operatorname{ad} a \mid n)
$$


for all $a \in$ a, i.e.,

$$
\rho=\frac{1}{2} \sum_{\phi \in \Sigma_{+}}\left(\operatorname{dim} g^{\phi}\right) \phi .
$$

For all $\nu \in a^{*}$, define the $g$-module $X^{\nu}$ to be the induced module $V^{\nu-\rho}$. As above, let $\pi$ be the one-dimensional representation of $m \oplus a \oplus n$ defined by $\nu$. Then $X^{\nu}$ can be interpreted as the twisted induced module induced by the one-dimensional ( $m \oplus a \oplus n$ )-module corresponding to $\pi$, in the sense of [2, \$5.2]. That is, for all $m \in \mathfrak{m}, a \in a$ and $n \in \mathbb{n}$, the trace of the action of $m+a+n$ on $g /(m \oplus a \oplus n)$ is $-\operatorname{tr}(\operatorname{ad} a \mid n)=-2 \rho(a)$. But we shall not need this fact.

The canonical linear isomorphism $\lambda: S(g) \rightarrow \mathcal{G}$ is defined by the formula

$$
\lambda\left(g_{1} \cdots g_{n}\right)=\frac{1}{n !} \sum_{\sigma} g_{\sigma(1)} \cdot: g_{\sigma(n)}
$$

for all $n \in \mathbf{Z}_{+}$and $g_{i} \in g$; here the product on the left is taken in $S(\mathrm{~g})$, the products on the right are taken in $\mathcal{G}$, and $\sigma$ ranges through the group of permutations of $\{1, \ldots, n\}$ (see $[2, \S 2.4]$ ). For all $g \in g$ and $n \in \mathbf{Z}_{+}, \lambda\left(g^{n}\right)=$ $g^{n}$. Also, $\lambda$ is a $g$-module isomorphism (see $[2, \S 2.4 .10]$ ).

Let $\bar{k}$ be a field extension of $k, \bar{g}=g \otimes_{k} \bar{k}, \bar{E}=\notin \otimes_{k} \bar{k}$, etc., and let $\bar{\theta}$ be the $\bar{k}$-linear extension of $\theta$ to $\bar{g}$. Then $(\bar{g}, \bar{\theta})$ is a semisimple symmetric Lie algebra over $\bar{k}$ with symmetric decomposition $\bar{g}=\bar{\xi} \oplus \bar{p}, \bar{a}$ is a splitting Cartan subspace of $\bar{p}$, etc. We shall often use the technique of extension to a "sufficiently large" field $\bar{k}$, which can always be taken to be an algebraic closure of $k$. For example, the construction of the subalgebra $u_{\phi}$ above might have to be carried out over an extension field $\bar{k}$ of $k$, but results about $(\bar{g}, \bar{\theta})$ proved using $\mathfrak{u}_{\phi}$ can often be transferred to $(g, \theta)$.

3. General results on polynomial invariants. Let $U$ be a finite-dimensional real Euclidean space and $S O(U)$ the rotation group of $U$. There is a natural $S O(U)$-invariant quadratic element $t$ of the second symmetric power $S^{2}\left(U^{*}\right)$ given by the sum of the squares of the members of the dual basis of any orthonormal basis of $U$ ( $t$ is the "square of the radius"). Let $I$ be the algebra of $S O(U)$-invariant polynomial functions on $U$, or equivalently, the algebra of $S O(U)$-invariants in the symmetric algebra $S\left(U^{*}\right)$. A standard result of classical invariant theory states that $I$ is exactly the set of polynomials in $t$ if $\operatorname{dim} U>1$. (If $\operatorname{dim} U=1$, then $S O(U$ ) acts trivially on $U$, and so $I=S\left(U^{*}\right)$.)

Clearly, $I$ is exactly the set of polynomial functions on $U$ constant on the $S O(U)$-orbits in $U$, i.e., the spheres centered at the origin if $\operatorname{dim} U>1$, and the points if $\operatorname{dim} U=1$. If $M$ is any Lie group which acts as isometries 
on $U$ in such a way that $M$ acts transitively on the $S O(U)$-orbits in $U$ (i.e., the $M$-orbits in $U$ are the same as the $S O(U)$-orbits), then the set of $M$-invariant polynomial functions on $U$ must coincide with the set $I$ of $S O(U)$-invariants.

Now suppose that $M$ also acts as isometries on a second finite-dimensional Euclidean space $V$ so that $M$ acts transitively on the $S O(V)$-orbits in $V$. Then the set of $M$-invariant polynomial functions on $V$ is the set $J \subset S\left(V^{*}\right)$ of $S O(V)$-invariants, and $J$ is a polynomial algebra as above.

Now $M$ and $S O(U) \times S O(V)$ both act naturally on $U \oplus V$. Let $L$ be the set of $M$-invariants in $S\left((U \oplus V)^{*}\right)=S\left(U^{*}\right) \otimes S\left(V^{*}\right)$. It is easy to see that the set of $S O(U) \times S O(V)$-invariants in $S\left((U \oplus V)^{*}\right)$ is exactly $I \otimes J$, and that $I \otimes J \subset L$. It is important to know that $I \otimes J=L$ in certain situations. In this case, for example, $L$ will be a polynomial algebra on two generators. In order to insure this, it is natural to assume that the $M$-orbits in $U \oplus V$ are the same as the $S O(U) \times S O(V)$-orbits, i.e., the products of the $S O(U)$-orbits in $U$ with the $S O(V)$-orbits in $V$. This assumption is equivalent to the "double-transitivity" hypothesis-that if $A$ is an $S O(U)$-orbit in $U$ and $B$ is an $S O(V)$-orbit in $V$, then the isotropy group of $M$ at any point of $A$ acts transitively on $B$. If $\operatorname{dim} U>1$ and $\operatorname{dim} V>1$, this is equivalent to saying that $M$ acts transitively on the product of the unit sphere in $U$ with the unit sphere in $V$. Under the double transitivity hypothesis, $L=I \otimes J$.

The present section is devoted to algebraic analogues of these facts, valid over the field $k$ of characteristic zero, assumed for convenience to be algebraically closed throughout this section. Here we are concerned with a Lie algebra $m_{0}$ (over $k$ ) which acts on modules $U$ and $V$ with nonsingular symmetric $m_{0}$-invariant bilinear forms. Replacing the orbit hypotheses for $M$ by corresponding "infinitesimal transitivity and double-transitivity" assumptions, we show that the $\mathrm{m}_{0}$-invariant polynomial functions on $U, V$ and $U \oplus V$ are exact analogues of the spaces of $M$-invariants above. We also transfer these results to the symmetric algebras $S(U), S(V)$ and $S(U \oplus V)=S(U) \otimes S(V)$; the invariants here are essentially the same as for the spaces of polynomial functions. We do not need any theory of algebraic groups. The setup in this section is entirely independent of $\$ 2$; the results here will be applied to the setting of $\$ 2$ in the next section.

Let $\mathrm{m}_{0}$ be a Lie algebra over $k, U$ a nonzero finite-dimensional $\mathrm{m}_{0}$-module, and $B_{0}$ a nonsingular symmetric $m_{0}$-invariant bilinear form on $U$. The homogeneous quadratic polynomial function $x \mapsto B_{0}(x, x)$ on $U$ defines a canonical nonzero element $t_{0} \in S^{2}\left(U^{*}\right)^{m} 0$ under the natural identification between the algebra of polynomial functions on $U$ and $S\left(U^{*}\right) . B_{0}$ also induces 
a canonical $\mathrm{m}_{0^{-}}$-module isomorphism $\xi_{0}: U^{*} \rightarrow U$ which extends to an $\mathrm{m}_{0^{-}}$ module and algebra isomorphism $\xi_{0}: S\left(U^{*}\right) \rightarrow S(U)$. Let $p_{0}=\xi_{0}\left(t_{0}\right)$, so that $p_{0} \in S^{2}(U)^{\mathrm{m}_{0}}$.

For every element $e \in U$, denote by $e^{\perp}$ the $B_{0}$-orthogonal complement of $e$ in $U$. Recall that $e$ is called isotropic (resp., nonisotropic) with respect to $B_{0}$ if $B_{0}(e, e)=0$ (resp., $B_{0}(e, e) \neq 0$ ). Note that $e$ is $B_{0}$-nonisotropic if and only if $U=k e \oplus e^{\perp}$.

Lemma 3.1. For all $e \in U, \mathrm{~m}_{0} \cdot e \subset e^{\perp}$.

Proof. Let $x \in \mathrm{m}_{0}$. Then $B_{0}(x \cdot e, e)=-B_{0}(e, x \cdot e)=-B_{0}(x \cdot e, e)$ since $B_{0}$ is $m_{0}$-invariant and symmetric, and so $B_{0}(x \cdot e, e)=0$. Q.E.D.

We now make the key assumption that for every $B_{0}$-nonisotropic vector $e \in U$, we have $\mathrm{m}_{0} \cdot e=e^{\perp}$. This can be thought of as an "infinitesimal transitivity" hypothesis. Our goal now is to compute $S(U)^{\mathrm{m}_{0}}$, and in fact to prove:

Theorem 3.2. If $\operatorname{dim} U=1$, then $S(U)^{\mathrm{m} 0}=S(U)$. If $\operatorname{dim} U \geq 2$, then $S(U)^{\mathrm{m} 0}$ is the polynomial algebra generated by $p_{0}$. In particular, $S(U)^{\mathrm{m}_{0}}$ is a polyno. mial algebra on one generator.

The proof will be carried out in a series of lemmas. First we settle the easy one-dimensional case:

Lemma 3.3. Suppose $\operatorname{dim} U=1$. Then $\mathrm{m}_{0}$ acts trivially on $U$. In particular, $S(U)^{\mathrm{m}}{ }^{0}=S(U)$.

Proof. Any nonzero element $e$ of $U$ is $B_{0}$-nonisotropic, and so $e^{\perp}=0$. Thus $\mathrm{m}_{0} \cdot e=0$ (Lemma 3.1). Q.E.D.

It is also convenient to handle the two-dimensional case separately:

Lemma 3.4. Suppose $\operatorname{dim} U=2$. Then $S(U)^{\mathrm{m}_{0}}$ is the polynomial algebra generated by $p_{0}$.

Proof. Since $k$ is algebraically closed, we may choose a $B_{0}$-orthonormal basis $\left\{e_{1}, e_{2}\right\}$ of $U$. Then $p_{0}=e_{1}^{2}+e_{2}^{2} \in S^{2}(U)$. By hypothesis, there exists $x \in \mathrm{m}_{0}$ such that $x \cdot e_{1}=e_{2}$. Since $B_{0}$ is $\mathrm{m}_{0}$-invariant, we have $B_{0}\left(e_{1}, x \cdot e_{2}\right)$ $=-B_{0}\left(x \cdot e_{1}, e_{2}\right)=-B_{0}\left(e_{2}, e_{2}\right)=-1$. But $x \cdot e_{2}$ is a multiple of $e_{1}$, and so $x \cdot e_{2}=-e_{1}$.

Again since $k$ is algebraically closed, $k$ contains a square root $i$ of -1. Let $v_{1}=e_{1}+i e_{2}$ and $v_{2}=e_{1}-i e_{2}$, so that $\left\{v_{1}, v_{2}\right\}$ is a basis of $U$. Then $x \cdot v_{1}=e_{2}-i e_{1}=-i v_{1}$ and $x \cdot v_{2}=e_{2}+i e_{1}=i v_{2}$.

Let $f \in S(U)^{\mathrm{m}_{0}}$. Then $f$ is a polynomial of the form 


$$
f=\sum_{a, \beta \in Z_{+}} c_{\alpha \beta} v_{1}^{a} v_{2}^{\beta}
$$

in $v_{1}$ and $v_{2}\left(c_{\alpha \beta} \in k\right)$. Since $x \cdot f=0$, we have

$$
\sum_{a, \beta \in Z_{+}} i c_{a \beta}(\beta-\alpha) v_{1}^{a} v_{2}^{\beta}=0,
$$

so that $c_{\alpha \beta}=0$ unless $a=\beta$. Thus $f=\Sigma_{a \in Z_{+}} c_{a \alpha}\left(v_{1} v_{2}\right)^{a}$. But $v_{1} v_{2}=e_{1}^{2}$ $+e_{2}^{2}=p_{0}$, and so $f$ is a polynomial in $p_{0}$. Conversely, it is clear that any polynomial in $p_{0}$ is in $S(U)^{\mathrm{m} 0}$. The lemma now follows from the fact that the subalgebra of $S(U)$ generated by $p_{0}$ is isomorphic to the polynomial algebra generated by $p_{0}$. Q.E.D.

In order to compute $S(U)^{\mathrm{m}} 0$ in general, we shall use the following result:

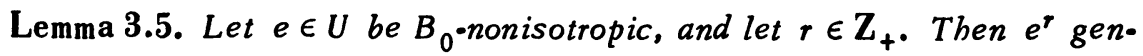
erates $S^{r}(U)$ as an $\mathrm{m}_{0}$-module. In particular,

$$
S^{r}(U)=k e^{r}+m_{0} \cdot S^{r}(U) .
$$

Proof. The second statement clearly follows from the first, and so it is sufficient to prove by induction on $j=0, \ldots, r$ that the smallest $m_{0}$-invariant subspace $T$ of $S^{r}(U)$ containing $e^{r}$ also contains $e^{r-j} S^{j}\left(e^{\perp}\right)$. This is clearly true for $j=0$, so assume it is true for $0, \ldots, j(j<r)$. Let $x \in \mathrm{m}_{0}$ and $s \in S^{j}\left(e^{\perp}\right)$. Then

$$
x \cdot e^{r-j} s=(r-j) e^{r-(j+1)}(x \cdot e) s+e^{r-j}(x \cdot s) .
$$

The left-hand side and the second term on the right are in $T$ by the induction hypothesis, and so $e^{r-(j+1)}(x \cdot e) s \in T$ since $r-j>0$. The lemma now follows from the assumption that $\mathrm{m}_{0} \cdot e=e^{\perp}$. Q.E.D.

The point is the following:

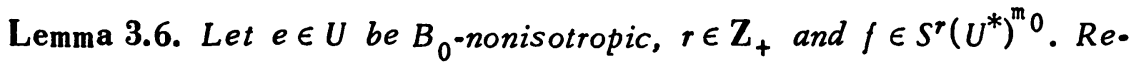
gard $f$ as a polynomial function on $U$. Then $f$ is determined by its value at e. Equivalently, if $f(e)=0$; then $f=0$.

Proof. There is a natural pairing $\{., .$,$\} between S^{r}\left(U^{*}\right)$ and $S^{r}(U)$ given as follows:

$$
\left\{f_{1} \cdots f_{r}, u_{1} \cdots u_{r}\right\}=\sum_{\sigma} \prod_{i=1}^{r}\left\langle f_{i}, u_{\sigma(i)}\right\rangle
$$

where $u_{1}, \ldots, u_{r} \in U, f_{1}, \ldots, f_{r} \in U^{*},\langle\cdot, \cdot\rangle$ is the natural pairing between $U^{*}$ and $U$ and $\sigma$ ranges through the group of permutations of $\{1, \ldots, r\}$. Then $\left\{f, u^{r}\right\}=r ! f(u)$ for all $f \in S^{r}\left(U^{*}\right)$ and $u \in U$, where $f$ is regarded as a 
polynomial function on $U$ on the right-hand side. It follows that $\{\cdot, \cdot$,$\} is$ nonsingular. Also, the natural actions of $m_{0}$ on $S^{r}\left(U^{*}\right)$ and $S^{r}(U)$ are contragredient with respect to $\{\cdot, \cdot \cdot\}$ (see for example the proof of $[7(b)$, Lemma 3.6]).

Now let $f$ and $e$ be as in the statement of the lemma. If $f(e)=0$, then $\left\{f, e^{r}\right\}=0$. Since $f$ is $m_{0}$-invariant, $\{f, x \cdot s\}=-\{x \cdot f, s\}=0$ for all $x \in \mathrm{m}_{0}$ and $s \in S^{r}(U)$. Thus $\left\{f, S^{r}(U)\right\}=0$ by Lemma 3.5 , and so $f=0$ by the nonsingularity of $\{\cdot, \cdot\}$. Q.E.D.

Theorem 3.2 now follows by applying the canonical isomorphism $\xi_{0}: S\left(U^{*}\right)$ $\rightarrow S(U)$ to the following result:

Lemma 3.7. Let $\operatorname{dim} U \geq 3$. Then $S\left(U^{*}\right)^{\mathrm{m}_{0}}$ is the polynomial algebra generated by $t_{0}$. Equivalently, if $r \in \mathbf{Z}_{+}$is odd, then $S^{r}\left(U^{*}\right)^{m} 0=0$, and if $r=$ $2 m, m \in \mathbf{Z}_{+}$, then $S^{r}\left(U^{*}\right)^{m_{0}}$ is spanned by $t_{0}^{m}$.

Proof. Since $S\left(U^{*}\right)^{m} 0$ is the direct sum of its homogeneous components, it is sufficient to compute $S^{r}\left(U^{*}\right)^{m} 0$ for $r \in \mathbf{Z}_{+}$. Let $V \subset U$ be the algebraic set defined by the equation $t_{0}(v)=0(v \in U)$. Then $V$ is exactly the set of $B_{0}$-isotropic vectors in $U$. Let $f \in S^{r}\left(U^{*}\right)^{m} 0$. If $f$ has a zero outside $V$, then $f=0$ by Lemma 3.6. Hence we may assume that all the zeros of $f$ lie in $V$. But then by the Hilbert Nullstellensatz, $f$ divides some power of $t_{0}$. Choose a $B_{0}$-orthonormal basis of $U$, and let $X_{1}, \ldots, X_{n} \in U^{*}$ be the corresponding dual basis. Then $S\left(U^{*}\right)$ can be identified with the polynomial algebra $k\left[X_{1}, \ldots, X_{n}\right]$, and $t_{0}=X_{1}^{2}+\cdots+X_{n}^{2}$. Since $\operatorname{dim} U \geq 3, t_{0}$ is an irreducible polynomial. The fact that $f$ divides a power of $t_{0}$ thus implies that $f$ is itself a power of $t_{0}$ up to a scalar multiple. Q.E.D.

Theorem 3.2 is now proved.

Remark. The last assertion of Lemma 3.7 (the case $r=2 \mathrm{~m}$ ) can also be proved more directly (even when $\operatorname{dim} U \leq 2$ ) as follows: Let $f \in S^{r}\left(U^{*}\right)^{m} 0$, let $e \in U$ be a $B_{0}$-nonisotropic vector, and set $c=\left(t_{0}^{m}\right)(e)=t_{0}(e)^{m} \in k$. Since $t_{0}(e)=B_{0}(e, e) \neq 0$, we have $c \neq 0$. But $f(e) t_{0}^{m}$ and $c f$ are two elements of $S^{r}\left(U^{*}\right)^{m_{0}}$ which take the same value $c f(e)$ at $e$. Hence $f=c^{-1} f(e) t_{0}^{m}$, by Lemma 3.6, proving the assertion.

The following consequence is interesting, but it will not be needed:

Corollary 3.8 (to Theorem 3.2). Every $m_{0}$-invariant symmetric bilinear form on $U$ is a scalar multiple of $B_{0}$.

Proof. From Theorem 3.2, $S^{2}(U)^{m_{0}}=k p_{0}$, and so $S^{2}\left(U^{*}\right)^{m_{0}}=k t_{0}$. The corollary now follows by polarization. Q.E.D. 
Remark. Corollary 3.8 has a direct proof which does not use either Lemma 3.4 or Lemma 3.6: Let $C$ be an $m_{0}$-invariant symmetric bilinear form on $U$. Then the unique linear operator $A: U \rightarrow U$ defined by $C(u, v)=B_{0}(A u, v)$ for all $u, v \in U$ is an $m_{0}$-module map which is symmetric with respect to $B_{0}$. Let $e \in U$ be a $B_{0}$-nonisotropic vector, and let $e^{\prime} \in e^{\perp}$. By hypothesis, there exists $x \in \mathrm{m}_{0}$ such that $x \cdot e=e^{\prime}$. Then

$$
\begin{aligned}
B_{0}\left(A e, e^{\prime}\right) & =B_{0}(A e, x \cdot e)=-B_{0}(x \cdot A e, e)=-B_{0}(A(x \cdot e), e) \\
& =-B_{0}\left(A e^{\prime}, e\right)=-B_{0}\left(e, A e^{\prime}\right)=-B_{0}\left(A e, e^{\prime}\right),
\end{aligned}
$$

and so $B_{0}\left(A e, e^{\prime}\right)=0$. Thus every $B_{0}$-nonisotropic vector of $U$ is an eigenvector for $A$. Since every two $B_{0}$-orthogonal $B_{0}$-nonisotropic vectors have a $B_{0}$-nonisotropic linear combination not proportional to either of them, we see that they must have the same eigenvalue for $A$. Applying this to a $B_{0}$-orthogonal basis of $U$ consisting of $B_{0}$-nonisotropic vectors shows that $A$ is a scalar, and this completes the proof.

Another general result is required for the next section. Let $V$ be a nonzero finite-dimensional $m_{0}$-module with a nonsingular symmetric $\mathfrak{m}_{0}$-invariant bilinear form $B_{1}$. Let $p_{1} \in S^{2}(V)^{\mathrm{m} 0}$ be the corresponding canonical invariant. The symmetric algebra of the direct sum $m_{0}$-module $U \oplus V$ is naturally isomorphic to $S(U) \otimes S(V)$, and $\mathrm{m}_{0}$ acts on $S(U \oplus V)$ according to the tensor product of its actions on $S(U)$ and $S(V)$. In particular, $S(U)^{\mathrm{m}_{0}} \otimes S(V)^{\mathrm{m} 0}$ $C S(U \oplus V)^{\mathrm{m} 0}$. The next theorem gives an important case in which this inclusion becomes an equality.

Theorem 3.9. In the context of Theorem 3.2, suppose in addition that for

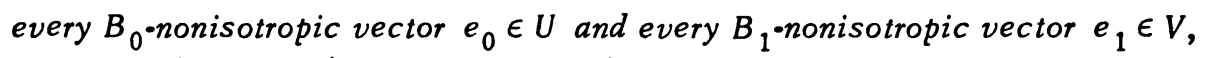
we have $\mathrm{m}_{0}^{\prime} \cdot e_{1}=e_{1}^{\frac{1}{1}}$ in $V$, where $\mathrm{m}_{0}^{\prime}$ is the centralizer of $e_{0}$ in $\mathrm{m}_{0}$. Then

$$
S(U \oplus V)^{\mathrm{m}_{0}}=S(U)^{\mathrm{m}_{0}} \otimes S(V)^{\mathrm{m}_{0}} \text {, }
$$

$S(U)^{\mathrm{m}_{0}}$ is given by Theorem 3.2, and $S(V)^{\mathrm{m}_{0}}$ is either $S(V)$ or the polynomial algebra generated by $p_{1}$, depending on whether $\operatorname{dim} V=1$ or $\operatorname{dim} V \geq 2$. In particular, $S(U \oplus V)^{\mathrm{m}} \mathrm{O}^{0}$ is a polynomial algebra on two generators.

Proof. Let $e_{0} \in U$ be $B_{0}$-nonisotropic, and let $\mathrm{m}_{0}^{\prime}$ be the centralizer of $e_{0}$ in $\mathrm{m}_{0}$. For every $B_{1}$-nonisotropic vector $e_{1} \in V$, we have $e_{1}^{\perp}=\mathrm{m}_{0}^{\prime} \cdot e_{1} C$ $\mathrm{m}_{0} \cdot e_{1} \subset e_{1}^{\perp}$ by Lemma 3.1 , so that $\mathrm{m}_{0} \cdot e_{1}=e_{1}^{\perp}$. Thus Theorem 3.2 applies to $\mathrm{m}_{0}, V, B_{1}$ and $p_{1}$, and so to prove the theorem all we must show is that $S(U \oplus V)^{\mathrm{m} 0} C S(U)^{\mathrm{m}_{0}} \otimes S(V)^{\mathrm{m} 0}$.

We shall now apply a technique used in $[7(b), \S 5]$. It is clear that 
$S(U \oplus V)^{\mathrm{m} 0}$ is the direct sum of its homogeneous components of the form $\left(S^{r}(U) \otimes S(V)\right)^{\mathrm{m} 0}$, where $r \in \mathbf{Z}_{+}$, and so it is sufficient to show that $\left(S^{r}(U) \otimes S(V)\right)^{\mathrm{m}} 0 C S^{r}(U)^{\mathrm{m}} 0 \otimes S(V)^{\mathrm{m}} 0$.

Recall the nonsingular $\mathfrak{m}_{0}$-invariant pairing $\{\cdot, \cdot\}$ between $S^{r}\left(U^{*}\right)$ and $S^{r}(U)$ (see the proof of Lemma 3.6). Also recall the canonical $m_{0}$-module and algebra isomorphism $\xi_{0}: S\left(U^{*}\right) \rightarrow S(U)$. Then $\xi_{0}$ restricts to an $m_{0}$-module isomorphism $\xi_{0}: S^{r}\left(U^{*}\right) \rightarrow S^{r}(U)$. Define a bilinear map

$$
\omega: S^{r}(U) \otimes S(V) \times S^{r}(U) \rightarrow S(V)
$$

by the condition $s \otimes w, t \mapsto\left\{\xi_{0}^{-1}(s), t\right\} w$ for all $s, t \in S^{r}(U)$ and $w \in S(V)$. Then for all $x \in \mathfrak{m}_{0}, y \in S^{r}(U) \otimes S(V)$ and $t \in S^{r}(U)$, we have

$$
\omega(x \cdot y, t)+\omega(y, x \cdot t)=x \cdot \omega(y, t) .
$$

Moreover, let $X$ be any subspace of $S(V)$. We claim that for all $y \in S^{r}(U) \otimes$ $S(V), \omega\left(y, S^{r}(U)\right) \subset X$ implies $y \in S^{r}(U) \otimes X$. In fact, choose a basis $\left\{w_{i}\right\}$ for a complement of $X$ in $S^{r}(V)$ and write $y=\Sigma_{i} s_{i} \otimes w_{i}+z\left(s_{i} \in S^{r}(U), z \epsilon\right.$ $\left.S^{r}(U) \otimes X\right)$. Then for all $t \in S^{r}(U)$, we have

$$
\sum_{i} \omega\left(s_{i} \otimes w_{i}, t\right)+\omega(z, t) \in X,
$$

and so $\Sigma_{i}\left\{\xi_{0}^{-1}\left(s_{i}\right), t\right\} w_{i} \in X$. Hence $\left\{\xi_{0}^{-1}\left(s_{i}\right), s^{r}(U)\right\}=0$ for all $i$, so that each $s_{i}=0$, proving the claim.

Let $y \in\left(S^{r}(U) \otimes S(V)\right)^{m_{0}}$, and let $e_{0}$ and $\mathrm{m}_{0}^{v}$ be as in the statement of the theorem. Then for all $x \in \mathfrak{m}_{0}^{\prime}$,

$$
x \cdot \omega\left(y, e_{0}^{r}\right)=\omega\left(x \cdot y, e_{0}^{r}\right)+\omega\left(y, r\left(x \cdot e_{0}\right) e_{0}^{r-1}\right)=0
$$

since $x \cdot y=0$ and $x \cdot e_{0}=0$. Hence $\omega\left(y, e_{0}^{r}\right) \in S(V)^{m_{0}^{\prime}}$. But by hypothesis, $\mathfrak{m}_{0}^{\prime} \cdot e_{1}=e_{1}^{\perp}$ in $V$, for every $B_{1}$-nonisotropic vector $e_{1} \in V$. Thus Theorem 3.2 applies, to $\mathrm{m}_{0}^{\prime}, V, B_{1}$ and $p_{1}$, as well as to $\mathrm{m}_{0}, V, B_{1}$ and $p_{1}$. In particular, $S(V)^{\mathrm{m}_{0}}=S(V)^{\mathrm{m}_{0}}$, and so $\omega\left(y, e_{0}^{r}\right) \in S(V)^{\mathrm{m}_{0}}$. But the set $Z$ of $B_{0}$-nonisotropic vectors in $U$ is Zariski dense since it is the set on which the polynomial function $t_{0} \in S^{2}\left(U^{*}\right)$ does not vanish. Hence the powers $e_{0}^{r}\left(e_{0} \in Z\right)$ span $S^{r}(U)$ (see for example [7(b), Lemma 3.5(ii)]). It follows that $\omega\left(y, S^{\gamma}(U)\right.$ ) $C S(V)^{\mathrm{m}_{0}}$. But now the above claim applied to $X=S(V)^{\mathrm{m} 0}$ implies that $y \in$ $S^{r}(U) \otimes S(V)^{m} 0$.

The rest is easy: Let $\left\{a_{i}\right\}$ be a basis of $S(V)^{\mathrm{m}_{0}}$, and write $y=\Sigma_{i} b_{i} \otimes$ $a_{i}\left(b_{i} \in S^{r}(U)\right)$. Since $\mathfrak{m}_{0} \cdot y=0$, we must have $\Sigma_{i} x \cdot b_{i} \otimes a_{i}=0$ for all $x \in \mathrm{m}_{0}$, so that $\mathrm{m}_{0} \cdot b_{i}=0$ for each $i$. Hence $y \in S^{r}(U)^{\mathrm{m}_{0}} \otimes S^{2}(V)^{\mathrm{m}_{0}}$, and the theorem is proved. Q.E.D. 
4. The Kostant-Mostow double transitivity theorem. In this section, we return to the setting of $\$ 2$. For every $\phi \in \Sigma, m$ acts naturally on the subalgebra $n_{\phi}=g^{\phi} \oplus g^{2 \phi}$ of $g$. (Here $g^{2 \phi}$ might be zero.) Our main goal at this point is to determine the algebra $S\left(n_{\phi}\right)^{\mathrm{m}}$ of m-invariants in the symmetric algebra $S\left(n_{\phi}\right)$. It will turn out to be a polynomial algebra on one or two generators (Theorem 4.6). The method will be to verify the hypotheses of $\$ 3$ and then to apply the results of $\$ 3$.

Suppose that $\operatorname{dim} a=1, \phi$ is the unique simple restricted root, $\operatorname{dim} g^{2 \phi}$ $>1, k=\mathbf{R}, \theta$ is a Cartan involution of $g$ in the sense that the Killing form of $g$ is negative definite on and positive definite on $\mathfrak{F}, G$ is a connected Lie group corresponding to $g, K$ is the connected Lie subgroup of $G$ corresponding to $€$, and $M$ is the centralizer of $a$ in $K$. Then $S\left(n_{\phi}\right)^{m}$ is the space $S\left(n_{\phi}\right)^{M}$ of $M$-invariants in $S\left(n_{\phi}\right)$, and determining $S\left(n_{\phi}\right)^{M}$ amounts to proving a double transitivity theorem for the action of $M$ on $g^{\phi} \oplus g^{2 \phi}$. Specifically, let $S_{1}$ be the unit sphere in $g^{\phi}$, and $S_{2}$ the unit sphere in $g^{2 \phi}$, with respect to the bilinear form $B_{\theta}$, which is positive definite on $g$. The issue is to prove that $M$ acts transitively on $S_{1} \times S_{2}$. This theorem was proved by $B$. Kostant $[6, \$ 2.1]$ (in a somewhat different formulation) and independently by G. D. Mostow (oral communication; related ideas are discussed in [8, \$19]). Kostant's proof, as modified slightly by N. Wallach [11, Theorem 8.11.3], is purely algebraic. In order to show that this proof applies in our general setting, and for our later reference, we shall give an exposition of Kostant's proof below. (Mostow's proof is based on explicit case-by-case checking; only the case of the exceptional group $F_{4}$ is difficult.) We have been discussing the rather subtle situation in which $\operatorname{dim} g^{2 \phi}>1$; if $\operatorname{dim} g^{2 \phi} \leq 1$, the appriate results are very easy.

Return now to the general setting of $\$ 2$.

Fix $\phi \in \Sigma$. We shall describe a canonical element $p_{\phi} \in S^{2}\left(g^{\phi}\right)^{\mathrm{m}}$. The symmetric bilinear form $B_{\theta}$ is nonsingular on $g^{\phi}$ (see $\$ 2$ ). Since $B_{\theta}$ is $t$-invariant and hence m-invariant, and since $g^{\phi}$ is m-stable, the restriction of $B_{\theta}$ to $g^{\phi}$ is m-invariant. As in $\$$, we get a nonzero homogeneous quadratic polynomial function $x \mapsto B_{\theta}(x, x)$ on $g^{\phi}$, and this defines a nonzero element $t_{\phi} \epsilon$ $s^{2}\left(\left(g^{\phi}\right)^{*}\right)^{\mathfrak{m}} \cdot B_{\theta}$ induces a canonical m-module isomorphism $\xi_{\phi}:\left(g^{\phi}\right)^{*} \rightarrow g^{\phi}$ which extends to an m-module and algebra isomorphism $\xi_{\phi}: S\left(\left(g^{\phi}\right)^{*}\right) \rightarrow S\left(g^{\phi}\right)$. Let $p_{\phi}=\xi_{\phi}\left(t_{\phi}\right)$, so that $p_{\phi} \in S^{2}\left(g^{\phi}\right)^{m}$.

Now we shall verify that the key assumption of the beginning of $\$ 3$ holds in the present context, with $m_{0}=m$ acting on $U=g^{\phi}$ by the adjoint action, and $B_{0}=B_{\theta} \mid g^{\phi} \times g^{\phi}$. The word "nonisotropic" and the symbol $e^{\perp}$ have the meanings of $\$ 3$. 
Lemma 4.1 (cf. [6, Theorem 2.1.7]). Let $e_{0} \in \mathrm{g}^{\phi}$ be a $B_{\theta^{-n o n i s o t r o p i c}}$ vector. Then $\left[\mathrm{m}, e_{0}\right]=e_{0}^{\frac{1}{0}}$. In particular, $g^{\phi}=k e_{0} \oplus\left[\mathrm{m}, e_{0}\right]$.

Proof. It is clearly sufficient to assume that $k$ is algebraically closed. As in $\$ 2$, we may choose a multiple $e_{\phi}$ of $e_{0}$ such that $B_{\theta}\left(e_{\phi}, e_{\phi}\right)=2 /(\phi, \phi)$. Setting $h_{\phi}=2 x_{\phi} /(\phi, \phi) \in a$ and $f_{\phi}=-\theta e_{\phi}$, we have the bracket relations $\left[h_{\phi}, e_{\phi}\right]=2 e_{\phi},\left[h_{\phi}, f_{\phi}\right]=-2 f_{\phi}$ and $\left[e_{\phi}, f_{\phi}\right]=h_{\phi}$ (see $\S 2$ ), so that $\left\{h_{\phi}\right.$, $\left.e_{\phi}, f_{\phi}\right\}$ spans a three-dimensional simple Lie subalgebra $u_{\phi}$ of $g$. Let $g_{\phi}$ be the $u_{\phi}$-submodule $\amalg_{j=-2}^{2} g^{j \phi}$ of $g$. Since the eigenspaces of ad $h_{\phi}$ in $g_{\phi}$ with eigenvalues 0 and 2 are $g^{0}=m \oplus a$ and $g^{\phi}$, respectively, the representation theory of a three-dimensional simple Lie algebra implies that $\left[e_{\phi}, \mathfrak{m} \oplus a\right]=g^{\phi}$. But $\left[e_{\phi}, \mathrm{m}\right] \subset e_{\phi}^{1}$ by Lemma 3.1, and since $\left[e_{\phi}, a\right]=k e_{\phi}$, we must have $\left[e_{\phi}, m\right]=e_{\phi}^{1}$. The lemma is now clear. Q.E.D.

Before applying Theorem 3.2, we shall derive two more results:

Lemma 4.2. We have $\left[g^{\phi}, g^{\phi}\right]=g^{2 \phi}$.

Proof. We may assume that $k$ is algebraically closed. As in $\$ 2$ (or the last proof), we have the three-dimensional simple Lie subalgebra $u_{\phi}$ of $g$ spanned by $h_{\phi}, e_{\phi}$ and $f_{\phi}$. Let $g_{\phi}$ be the $u_{\phi}$-submodule $\amalg_{j=-2}^{2} g^{j \phi}$ of $g$. The eigenspaces of $h_{\phi}$ in $g_{\phi}$ with eigenvalues 2 and 4 are $g^{\phi}$ and $g^{2 \phi}$, respectively, and so the representation theory of $u_{\phi}$ implies that $\left[e_{\phi}, g^{\phi}\right]=$ $g^{2 \phi}$. Q.E.D.

The following consequence will be useful later:

Corollary 4.3. Let $X$ be a g-module and $x \in X$ an m-invariant vector annihilated by some $B_{\theta}$-nonisotropic vector $e_{0} \in g^{\phi}$. Then $\left(g^{\phi} \oplus g^{2 \phi}\right) \cdot x=0$. In particular, if $\operatorname{dim} a=1$ and $\phi$ is the unique simple restricted root, then $x$ is a conical vector in $X$.

Proof. For all $y \in \mathrm{m},\left[y, e_{0}\right] \cdot x=y \cdot\left(e_{0} \cdot x\right)-e_{0} \cdot(y \cdot x)=0$, and so $g^{\phi} \cdot x=0$ by Lemma 4.1. Lemma 4.2 now implies that $g^{2 \phi} \cdot x=0$. The last assertion is clear. Q.E.D.

Theorem 3.2, Lemma 4.1 and the field extension technique imply:

Theorem 4.4. If $\operatorname{dim} g^{\phi}=1$, then $S\left(g^{\phi}\right)^{m}=S\left(g^{\phi}\right)$. If $\operatorname{dim} g^{\phi} \geq 2$, then $S\left(g^{\phi}\right)^{m}$ is the polynomial algebra generated by $p_{\phi}$. In particular, $S\left(g^{\phi}\right)^{\mathrm{m}}$ is a polynomial algebra on one generator.

Corollary 4.5. Every m-invariant symmetric bilinear form on $g^{\phi}$ is a scalar multiple of $B_{\theta}$.

The corollary follows from either Theorem 4.4 or Corollary 3.8; see the 
remark following Corollary 3.8 for a simple proof. We shall not have to use Corollary 4.5.

Our next goal is to verify the hypothesis of Theorem 3.9 for $U=g^{\phi}$ and $V=g^{2 \phi}$ in case $2 \phi \in \Sigma$ (see Lemma 4.7). This amounts to proving the Kostant-Mostow double transitivity theorem. For reasons mentioned above, we shall essentially repeat Kostant's proof $[6, \$ 2.1]$, with a couple of modifications (the proofs of Lemmas 4.18 and 4.20) taken from Wallach's exposition [11, Theorem 8.11.3]. The result is:

Theorem 4.6. Suppose $\phi \in \Sigma$, and let $n_{\phi}$ be the subalgebra $g^{\phi} \oplus g^{2 \phi}$ of g. Then $S\left(n_{\phi}\right)^{\mathrm{m}}=S\left(g^{\phi}\right)^{\mathrm{m}} \otimes S\left(g^{2 \phi}\right)^{\mathrm{m}}$, and this is a polynomial algebra. Moreover, let $p_{\phi} \in S^{2}\left(g^{\phi}\right)^{\mathrm{m}}$ be the canonical quadratic m-invariant defined by $B_{\theta}$, and if $2 \phi \in \Sigma$, let $p_{2 \phi} \in S^{2}\left(g^{2 \phi}\right)^{m}$ be the same for $2 \phi$. Then there are four possibilities:

Case 1. $\operatorname{dim} g^{\phi}=1$ and $g^{2 \phi}=0$. Let $x \in g^{\phi}, x \neq 0$. Then $S\left(n_{\phi}\right)^{\mathrm{m}}=$ $S\left(g^{\phi}\right)=k[x]$, and $k[x]$ is the polynomial algebra generated by $x$.

Case 2. $\operatorname{dim} g^{\phi}>1$ and $g^{2 \phi}=0$. Then $s\left(n_{\phi}\right)^{m}=k\left[p_{\phi}\right]$, and $k\left[p_{\phi}\right]$ is the polynomial algebra generated by $p_{\phi}$.

Case 3. $\operatorname{dim} g^{\phi}>\operatorname{dim} g^{2 \phi}=1$. Then $S\left(g^{\phi}\right)^{\mathrm{m}}=k\left[p_{\phi}\right]$ and $S\left(g^{2 \phi}\right)^{\mathrm{m}}=$ $S\left(g^{2 \phi}\right)=k[y]$, where $y \in g^{2 \phi}, y \neq 0$. Both algebras are polynomial algebras in the indicated generators, so that $S\left(n_{\phi}\right)^{\mathrm{m}}$ is the polynomial algebra $k\left[p_{\phi}, y\right]$ in the two generators $p_{\phi}$ and $y$.

Case 4. $\operatorname{dim} g^{\phi}>\operatorname{dim} g^{2 \phi}>1$. Then $S\left(g^{\phi}\right)^{\mathrm{m}}$ and $S\left(g^{2 \phi}\right)^{\mathrm{m}}$ are the poly. nomial algebras $k\left[p_{\phi}\right]$ and $k\left[p_{2 \phi}\right]$, respectively, so that $S\left(n_{\phi}\right)^{\mathrm{m}}$ is the poly. nomial algebra $k\left[p_{\phi}, p_{2 \phi}\right]$.

Proof. We may, and do, assume that $k$ is algebraically closed. The fact that $\operatorname{dim} g^{\phi}>\operatorname{dim} g^{2 \phi}$ will be proved in Lemina 4.8. Also, Cases 1 and 2 are covered in Theorem 4.4. The rest of Theorem 4.6 follows immediately from Theorem 3.9, Lemma 4.1 and:

Lemma 4.7. Suppose $\phi, 2 \phi \in \Sigma$. Let $e_{0} \in g^{\phi}$ and $e_{1} \in g^{2 \phi}$ be $B_{\theta}$-nonisotropic, and let $\mathrm{m}_{0}$ be the centralizer of $e_{0}$ in $\mathrm{m}$. Then $\left[\mathrm{m}_{0}, e_{1}\right]=e_{1}^{\perp}$ in $g^{2 \phi}$.

This result will follow from the next series of lemmas. Note that only Case 4 of Theorem 4.6 remains to be proved, since Lemma 4.7 is trivial if $\operatorname{dim} g^{2 \phi}=1$. But it will not be necessary in the following proof to impose any restriction on $\operatorname{dim} g^{2 \phi}$, and in fact the proof holds even if $g^{2 \phi}=0$.

We shall use the notation of the proof of Lemma 4.1, so that $e_{\phi}$ is a certain multiple of $e_{0}$, and $\left\{h_{\phi}, e_{\phi}, f_{\phi}\right\}$ spans a three-dimensional simple 
subalgebra $u_{\phi}$ of $g$. Also as in the proof of Lemma 4.1, let $g_{\phi}$ be the $u_{\phi^{-}}$ submodule $\amalg_{j=-2}^{2} g^{j \phi}$ of $g$. The natural representation of $u_{\phi}$ on $g_{\phi}$ decomposes $g_{\phi}$ into a direct sum of irreducible $u_{\phi}$-submodules. Since the eigenvalues of ad $h_{\phi}$ on $g_{\phi}$ are among $0, \pm 2$ and \pm 4 (with corresponding eigenspaces $g^{0}=\mathfrak{m} \oplus a, g^{ \pm \phi}$ and $g^{ \pm 2 \phi}$, the dimensions of the irreducible components can only be 1, 3 and 5. A five-dimensional irreducible module occurs if and only if $g^{2 \phi} \neq 0$, and a three-dimensional irreducible module always occurs- $u_{\phi}$ itself. Let $g_{i} \subset g_{\phi}$ be the sum of all the $(2 i+1)$-dimensional irreducible $u_{\phi}$-submodules of $g_{\phi}(i=0,1,2)$, so that $g_{\phi}=g_{0} \oplus g_{1} \oplus g_{2}$. Also, let $g_{i}^{j}=g_{i} \cap g^{j \phi}(0 \leq i \leq 2,-2 \leq j \leq 2)$; then $g_{i}=\amalg_{j=-i}^{i} g_{i}^{j}$ for each $i=0,1,2$. Also, $g^{ \pm 2 \phi}=g_{2}^{ \pm 2}, g^{ \pm \phi}=g_{1}^{ \pm 1} \oplus g_{2}^{ \pm 1}$ and $g^{0}=g_{0}^{0} \oplus g_{1}^{0} \oplus g_{2}^{0}$.

Lemma 4.8. We have $\operatorname{dim} g^{\phi}>\operatorname{dim} g^{2 \phi}$.

Proof. This is clear since $g^{\phi}=g_{1}^{1} \oplus g_{2}^{1}, g^{2 \phi}=g_{2}^{2}$, dim $g_{2}^{1}=\operatorname{dim} g_{2}^{2}$ and $\operatorname{dim} g_{1}^{1} \geq 1$ (since $e_{\phi} \in g_{1}^{1}$ ). Q.E.D.

Lemma 4.9. The decomposition $g_{\phi}=g_{0} \oplus g_{1} \oplus g_{2}$ is both $B_{\theta^{-o r t h o g o n a l}}$ and B-orthogonal.

Proof. First we shall show that $B_{\theta}\left(g_{1}^{1}, g_{2}^{1}\right)=0$. Let $x \in g_{1}^{1}, y \in g_{2}^{1}$. Then $y=\left[f_{\phi}, z\right]$. for some $z \in g^{2 \phi}=g_{2}^{2}$, and so

$$
B_{\theta}(x, y)=-B(x, \theta y)=-B\left(x,\left[-e_{\phi}, \theta z\right]\right)=-B\left(\left[e_{\phi}, x\right], \theta z\right)=0
$$

since $\left[e_{\phi}, x\right]=0$. Hence $B_{\theta}\left(g_{1}^{1}, g_{2}^{1}\right)=0$, and similar arguments show that

$$
B_{\theta}\left(g_{1}^{-1}, g_{2}^{-1}\right)=B_{\theta}\left(g_{0}^{0}, g_{1}^{0}\right)=B_{\theta}\left(g_{0}^{0}, g_{2}^{0}\right)=0
$$

and

$$
B\left(g_{1}^{1}, g_{2}^{-1}\right)=B\left(g_{1}^{-1}, g_{2}^{1}\right)=B\left(g_{0}^{0}, g_{0}^{1}\right)=B\left(g_{0}^{0}, g_{0}^{2}\right)=0 \text {. }
$$

Since $B_{\theta}\left(g^{j \phi}, g^{k \phi}\right)=0$ unless $j=k$, and $B\left(g^{j \phi}, g^{k \phi}\right)=0$ unless $j=-k$, all that remains is to show that $B_{\theta}\left(g_{1}^{0}, g_{2}^{0}\right)=B\left(g_{1}^{0}, g_{2}^{0}\right)=0$. Let $u \in g_{1}^{0}, v \in g_{2}^{0}$. Then $v=\left[f_{\phi}, w\right]$ for some $w \in g_{2}^{1}$, so that

$$
\begin{aligned}
B_{\theta}(u, v) & =-B(u, \theta v)=-B\left(u,\left[-e_{\phi}, \theta w\right]\right) \\
& =-B\left(\left[e_{\phi}, u\right], \theta w\right)=B_{\theta}\left(\left[e_{\phi}, u\right], w\right)=0
\end{aligned}
$$

by the above, since $\left[e_{\phi}, u\right] \in g_{1}^{1}$ and $w \in g_{2}^{1}$. Thus $B_{\theta}\left(g_{1}^{0}, g_{2}^{0}\right)=0$. Similarly, $B\left(g_{1}^{0}, g_{2}^{0}\right)=0$. Q.E.D.

Lemma 4.10. Let $e \in g^{\phi}$ and $f \in g^{-\phi}$, and suppose $B(e, f)=0$, or equivalently, $B_{\theta}(f, \theta e)=0$ or $B_{\theta}(e, \theta f)=0$. Then $[e, f] \in \mathrm{m}$. 
Proof. Since $[e, f] \in g^{0}=\mathfrak{m} \oplus a$ and since $m$ is the $B$-orthogonal complement of $a$ in $g^{0}$, it is sufficient to show that $B([e, f], a)=0$. But if $b \in a$, then

$$
B([e, f], h)=-B(e,[h, f])=\phi(h) B(e, f)=0,
$$

and so the lemma is proved. Q.E.D.

Lemma 4.11. We have $g_{2}^{0} \subset \mathrm{m}$.

Proof. Every element in $g_{2}^{0}$ is of the form $\left[e_{\phi}, f\right]$, where $f \in g_{2}^{-1}$. Since $e_{\phi} \in g_{1}$, Lemma 4.9 implies that $B\left(e_{\phi}, f\right)=0$. But then $\left[e_{\phi}, f\right] \in \mathrm{m}$ by Lemma 4.10. Q.E.D.

Lemma 4.12. We have $g_{1}^{0}=k h_{\phi} \oplus\left(g_{1}^{0} \cap \mathrm{m}\right)$.

Proof. It is sufficient to show that $g_{1}^{0} \subset k h_{\phi}+m$. But $g_{1}^{0}=\left[e_{\phi}, g_{1}^{-1}\right]$ and $g_{1}^{-1} \subset g^{-\phi}=k f_{\phi} \oplus f_{\phi}^{1}$, where $f_{\phi}^{1}$ is the $B_{\theta}$-orthogonal complement of $f_{\phi}$ in $g^{-\phi}$. In fact, $B_{\theta}\left(f_{\phi}, f_{\phi}\right)=B_{\theta}\left(e_{\phi}, e_{\phi}\right) \neq 0$. Hence $g_{1}^{0} \subset k h_{\phi}+\left[e_{\phi}, f_{\phi}^{1}\right]$, and $\left[e_{\phi}, f_{\phi}^{1}\right] \subset m$ by Lemma 4.10. Q.E.D.

Lemma 4.13. We have $g_{0}^{0}=\operatorname{Ker} \phi \oplus\left(g_{0}^{0} \cap \mathrm{m}\right)$.

Proof. Since $g_{0}^{0}=g_{0}$ is the centralizer of $\mathfrak{u}_{\phi}$ in $g_{\phi}, g_{0}^{0}$ is stable under $\theta$, and so $g_{0}^{0}=\left(g_{0}^{0} \cap a\right) \oplus\left(g_{0}^{0} \cap m\right)$. But the centralizer of $u_{\phi}$ in $a$ is clearly $\operatorname{Ker} \phi$, and so $g_{0}^{0} \cap a=\operatorname{Ker} \phi$. Q.E.D.

Let $\mathfrak{m}_{i}=g_{i} \cap \mathrm{m}=g_{i}^{0} \cap \mathrm{m}(i=1,2,3)$, and note that $\mathfrak{m}_{0}$ is the centralizer of $e_{\phi}$ in $m$ and hence coincides with the subalgebra $m_{0}$ in the statement of Lemma 4.7. The next lemma summarizes the last three:

Lemma 4.14. We have $g_{2}^{0}=m_{2}, g_{1}^{0}=k h_{\phi} \oplus m_{1}$ and $g_{0}^{0}=\operatorname{Ker} \phi \oplus m_{0}$. In particular, $m=m_{0} \oplus m_{1} \oplus m_{2}$.

For all $x \in g$, define $x^{*}=\left[e_{\phi}, x\right]$. Write $x^{* *}$ instead of $\left(x^{*}\right)^{*}$. Also, define $x_{*}=\left[f_{\phi}, x\right]$, and write $x_{* *}$ for $\left(x_{*}\right)_{*}$.

Recall the following standard fact about the representation theory of the three-dimensional simple Lie algebra $u_{\phi}$ : Let $\pi$ be a finite-dimensional irreducible representation of $\mathfrak{u}_{\phi}$ on the space $V$ and let $v \in V$ be a nonzero eigenvector for $\pi\left(h_{\phi}\right)$. Let $p$ be the smallest nonnegative integer $j$ such that $\pi\left(f_{\phi}\right)^{j+1}(v)=0$ and $q$ the smallest nonnegative integer $j$ such that $\pi\left(e_{\phi}\right)^{j+1}(v)=0$. Then $\pi\left(f_{\phi}\right) \pi\left(e_{\phi}\right)(v)=(p+1) q v$ and $\pi\left(e_{\phi}\right) \pi\left(f_{\phi}\right)(v)=$ $(q+1) p v$. This implies:

Lemma 4.15. For all $x \in \mathrm{m}_{2},\left(x^{* *}\right)_{*}=4 x^{*},\left(x^{*}\right)_{*}=6 x,\left(x_{* *}\right)^{*}=4 x_{*}$ and $\left(x_{*}\right)^{*}=6 x$. 
Lemma 4.16. Let $x, y \in \mathrm{m}_{2}$. Then $\left[x, y^{* *}\right]=\left[x^{* *}, y\right]=(2 / 3)\left[y^{*}, x^{*}\right]$.

Proof. By Lemma 4.15,

$$
\left[x, y^{* *}\right]=(1 / G)\left[\left(x^{*}\right)_{*}, y^{* *}\right]=(1 / 6)\left[\left(y^{* *}\right)_{*}, x^{*}\right]=(2 / 3)\left[y^{*}, x^{*}\right] .
$$

Hence also

$$
\left[x^{* *}, y\right]=-\left[y, x^{* *}\right]=-(2 / 3)\left[x^{*}, y^{*}\right]=(2 / 3)\left[y^{*}, x^{*}\right] \text {. Q. E. D. }
$$

Lemma 4.17. For all $x, y \in \mathrm{m}_{2},[x, y]^{* *}=(2 / 3)\left[x^{*}, y^{*}\right]$.

Proof. We have

$$
[x, y]^{* *}=\left[x^{* *}, y\right]+2\left[x^{*}, y^{*}\right]+\left[x, y^{* *}\right]=(2 / 3)\left[x^{*}, y^{*}\right]
$$

by Lemma 4.16. Q.E.D.

For all $x \in g_{\phi}$, let $x_{i}(i=0,1,2)$ be the component of $x$ in $g_{i}$ with respect to the decomposition $g_{\phi}=g_{0} \oplus g_{1} \oplus g_{2}$.

Lemma 4.18. For all $x, y \in \mathfrak{m}_{2},[x, y]_{1}=0$.

Proof. By Lemma 4.17, $[x, y]^{* *}=(2 / 3)\left[x^{*}, y^{*}\right]$, so that

$$
\begin{aligned}
\left([x, y]^{* *}\right)_{*} & =(2 / 3)\left[\left(x^{*}\right)_{*}, y^{*}\right]+(2 / 3)\left[x^{*},\left(y^{*}\right)_{*}\right] \\
& =4\left[x, y^{*}\right]+4\left[x^{*}, y\right]=4[x, y]^{*},
\end{aligned}
$$

using Lemma 4.15. But $[x, y]^{* *}=\left([x, y]_{2}\right)^{* *}$, and $\left(\left([x, y]_{2}\right)^{* *}\right)_{*}=4\left([x, y]_{2}\right)^{*}$ by Lemma 4.15. Hence $[x, y]^{*}=\left([x, y]_{2}\right)^{*}$. But since $[x, y]^{*}=\left([x, y]_{1}\right)^{*}+$ $\left([x, y]_{2}\right)^{*}$, we get $\left([x, y]_{1}\right)^{*}=0$, and so $[x, y]_{1}=0$. Q.E.D.

Lemma 4.19. For all $x, y \in \mathrm{m}_{2},\left[[x, y]_{0}, y^{* *}\right]=-2\left[[x, y]_{2}, y^{* *}\right]$.

Proof. By Lemma 4.16, we have

$$
\left[[x, y]_{2}, y^{* *}\right]=\left[\left([x, y]_{2}\right)^{* *}, y\right]=\left[[x, y]^{* *}, y\right]=-\left[\left[x, y^{* *}\right], y\right]
$$

(Lemmas 4.16 and 4.17)

$$
=-\left[[x, y], y^{* *}\right]-\left[x,\left[y^{* *}, y\right]\right]=-\left[[x, y], y^{* *}\right]
$$

(Lemma 4.16)

$$
=-\left[[x, y]_{0}, y^{* *}\right]-\left[[x, y]_{2}, y^{* *}\right],
$$

by Lemma 4.18. The lemma now follows. Q.E.D. If $x \in \mathrm{m}$, note that $x_{*}=-\theta x^{*}$ and $x_{* *}=\theta x^{* *}$.

Lemma 4.20. Let $x, y \in \mathrm{m}_{2}$, and suppose $B_{\theta}\left(x^{* *}, y^{* *}\right)=0$. Then 
$\left[x^{* *}, y_{* *}\right] \in \mathrm{m},\left[x^{* *}, y_{* *}\right]=-\left[y^{* *}, x_{* *}\right]$, and $\left.\left[x^{* *}, y_{* *}\right]\right]_{1}=0$.

Proof. By Lemma 4.10 applied to $g^{2 \phi}$ in place of $g^{\phi}, e=x^{* *}$ and $f=$ $y_{* *}=\theta y^{* *}$, we have $\left[x^{* *}, y_{* *}\right] \in \mathrm{m}$. Thus

$$
\left[x^{* *}, y_{* *}\right]=\theta\left[x^{* *}, y_{* *}\right]=\left[\theta x^{* *}, \theta y_{* *}\right]=\left[x_{* *}, y^{* *}\right]=-\left[y^{* *}, x_{* *}\right],
$$

proving the second assertion.

To prove the last, first note that $\left(y_{* *}\right)^{*}=4 y_{*}$, by Lemma 4.15 . Hence

$$
\begin{aligned}
{\left[x^{* *}, y_{* *}\right]^{*} } & =\left[x^{* *},\left(y_{* *}\right)^{*}\right]=4\left[x^{* *}, y_{*}\right] \\
& =4\left[x^{* *}, y\right]_{*}-4\left[\left(x^{* *}\right)_{*}, y\right]=4\left[x^{* *}, y\right]_{*}-16\left[x^{*}, y\right]
\end{aligned}
$$

(again by Lemma 4.15)

$$
=-4\left([x, y]^{* *}\right)_{*}-16\left[x^{*}, y\right],
$$

by Lemmas 4.16 and 4.17. Thus

$$
\left(\left[x^{* *}, y_{* *}\right]_{1}\right)^{*}=-16\left[x^{*}, y\right]_{1} \text {. }
$$

Hence by the second assertion, we also have

$$
\left(\left[x^{* *}, y_{* *}\right]_{1}\right)^{*}=-\left(\left[y^{* *}, x_{* *}\right]_{1}\right)^{*}=16\left[y^{*}, x\right]_{1}=-16\left[x, y^{*}\right]_{1} \text {. }
$$

Thus

$$
\left(\left[x^{* *}, y_{* *}\right]_{1}\right)^{*}=-8\left(\left[x^{*}, y\right]+\left[x, y^{*}\right]\right)_{1}=-8\left([x, y]^{*}\right)_{1}=-8\left([x, y]_{1}\right)^{*}=0 \text {, }
$$

by Lemma 4.18. It is finally clear that $\left[x^{* *}, y_{* *}\right]_{1}=0$. Q.E.D.

Lemma 4.21. Let $x, y \in \mathrm{m}_{2}$, and suppose $B_{\theta}\left(x^{* *}, y^{* *}\right)=0$. Then $\left[x^{*}, y_{* *}\right]=-6[x, y]_{*}$.

Proof. We have $\left[x_{*}, y^{* *}\right]=(1 / 4)\left[x_{* *}, y^{* *}\right]^{*}$ by Lemma 4.15 , and this is $(1 / 4)\left[x^{* *}, y_{* *}\right]^{*}$ by Lemma 4.20. But $\left[x^{* *}, y_{* *}\right] \in \mathrm{m}$ (Lemma 4.20). Thus the last assertion of Lemma 4.20 shows that $\left[x_{*}, y^{* *}\right] \in g_{2}^{1}$. Now

$$
\left[x_{*}, y^{* *}\right]^{*}=\left[\left(x_{*}\right)^{*}, y^{* *}\right]=6\left[x, y^{* *}\right]
$$

(by Lemma 4.15)

$$
=-G[x, y]^{* *}
$$

by Lemmas 4.16 and 4.17. But both $\left[x_{*}, y^{* *}\right]$ and $[x, y]^{*}$ are in $g_{2}^{1}$ by the above and Lemma 4.18. Hence $\left[x_{*}, y^{* *}\right]=-G[x, y]^{*}$, and the lemma follows by applying $\theta$. Q.E.D.

Lemma 4.22. Let $x, y \in \mathrm{m}_{2}$, and suppose $B_{\theta}\left(x^{* *}, y^{* *}\right)=0$ and $B_{\theta}\left(y^{* *}, y^{* *}\right)=1 / 2(\phi, \phi)$. Then 


$$
\left[[x, y]_{0}, y^{* *}\right]=-x^{* *} / 18 \text {. }
$$

Proof. By Lemma 4.19, $\left[[x, y]_{0}, y^{* *}\right]=-2\left[[x, y]_{2}, y^{* *}\right]_{\text {. But }}[x, y]_{2}=$ $(1 / 6)\left([x, y]_{*}\right)^{*}$, by Lemmas 4.15 and 4.18 , and so

$$
\begin{aligned}
{\left[[x, y]_{0}, y^{* *}\right] } & =-(1 / 3)\left[\left([x, y]_{*}\right)^{*}, y^{* *}\right] \\
& =-(1 / 3)\left[[x, y]_{*}, y^{* *}\right]^{*}=(1 / 18)\left[\left[x^{*}, y_{* *}\right], y^{* *}\right]^{*},
\end{aligned}
$$

by Lemma 4.21. Also,

$$
B_{\theta}\left(y^{* *}, y^{* *}\right)=1 / 2(\phi, \phi)=2 /(2 \phi, 2 \phi),
$$

and so as in $\$ 2$ we must have the bracket relations for a three-dimensional simple Lie algebra, say $u_{2 \phi}$, spanned by $h_{2 \phi}, y^{* *}$ and $-\theta y^{* *}$ :

$$
\left[h_{2 \phi}, y^{* *}\right]=2 y^{* *},\left[h_{2 \phi},-\theta y^{* *}\right]=2 \theta y^{* *} \text { and }\left[y^{* *},-\theta y^{* *}\right]=h_{2 \phi} .
$$
But $-\theta y^{* *}=-y_{* *}$ and $h_{2 \phi}=1 / 2 h_{\phi}$. Thus $x^{*}$ is an eigenvector for ad $h_{2 \phi}$ with eigenvalue 1 , and must lie in a two-dimensional irreducible $u_{2 \phi}$-submodule of $\mathrm{g}$. Hence applying the discussion preceding Lemma 4.15 to $u_{2 \phi}$, we get

$$
\left[y^{* *},\left[-y_{* *}, x^{*}\right]\right]=x^{*} .
$$

Thus $\left[[x, y]_{0}, y^{* *}\right]=-x^{* *} / 18$, and the lemma is proved. Q.E.D.

In the notation of Lemma 4.7, a multiple $e^{\prime}$ of the nonisotropic vector $e_{1} \in g^{2 \phi}$ may be chosen so that $B\left(e^{\prime}, e^{\prime}\right)=1 / 2(\phi, \phi)$. Then $e^{\prime}$ is of the form $y^{* *}$ for some $y \in \mathrm{m}_{2}$. Let $e^{\prime \prime} \in e_{1}^{\perp}$. Then $e^{\prime \prime}=x^{* *}$ for some $x \in \mathrm{m}_{2}$, and so by Lemma $4.22,\left[-18[x, y]_{0}, e^{\prime}\right]=e^{\prime \prime}$. Thus there exists $z \in \mathfrak{m}_{0}$ such that $\left[z, e_{1}\right]=e^{\prime \prime}$. Lemma 4.7 is finally proved, and hence so is Theorem 4.6. Q.E.D.

5. The structure of $\tau_{\phi}^{m}$. Continuing to work in the setting of $\$ 2$, we shall transfer Theorem 4.6 to its "noncommutative analogue", i.e., to the structure theorem for $\tau_{\phi}^{m}$ (see below).

Retain the notation of $\$ 4$. In particular, $\phi \in \Sigma$ is fixed. Recall the canonical linear isomorphism $\lambda: S(g) \rightarrow \mathcal{G}$. Let $\pi_{\phi}$ be the universal enveloping algebra of the Lie subalgebra $n_{\phi}=g^{\phi} \oplus g^{2 \phi}$ of $g$ defined in Theorem 4.6 , so that $\lambda: S\left(n_{\phi}\right) \rightarrow \pi_{\phi}$ is an m-module isomorphism which restricts to a linear isomorphism from $S\left(n_{\phi}\right)^{\mathrm{m}}$ to $\pi_{\phi}^{\mathrm{m}}$. We shall now use Theorem 4.6 to give an explicit description of the algebra $\pi_{\phi}^{m}$. Recall the canonical quadratic m-invariants $p_{\phi} \in S^{2}\left(g^{\phi}\right)^{m}$ and (if $\left.2 \phi \in \Sigma\right) p_{2 \phi} \in S^{2}\left(g^{2 \phi}\right)^{m}$. Define

$$
q_{\phi}=2 \lambda\left(p_{\phi}\right) /(\phi, \phi) \in \Re_{\phi}^{\mathrm{m}},
$$


and similarly, if $2 \phi \in \Sigma$, define

$$
q_{2 \phi}=2 \lambda\left(p_{2 \phi}\right) /(2 \phi, 2 \phi)=\lambda\left(p_{2 \phi}\right) / 2(\phi, \phi) \in \pi_{\phi}^{m} .
$$

Theorem 5.1. $\tau_{\phi}^{\mathrm{m}}$ is commutative and in fact is a polynomial algebra. More precisely, in the four cases of Theorem 4.6, we have:

Case 1. $\pi_{\phi}^{m}=\pi_{\phi}=k[x]$, the polynomial algebra generated by an arbi. trary nonzero $x \in g^{\phi}$.

Case 2. $r_{\phi}^{m}=k\left[q_{\phi}\right]$, the polynomial algebra generated by $q_{\phi}$.

Case 3. $\pi_{\phi}^{m}=k\left[q_{\phi}, y\right]$, where $y$ is an arbitrary nonzero element of $\mathrm{g}^{2 \phi}$; this is the polynomial algebra in the indicated generators.

Case $4 . \pi_{\phi}^{m}=k\left[q_{\phi}, q_{2 \phi}\right]$, the polynomial algebra generated by $q_{\phi}$ and $q_{2 \phi}$

Proof. Cases 1 and 2 follow immediately from the corresponding cases of Theorem 4.6, together with the fact that $\lambda: S\left(n_{\phi}\right) \rightarrow \pi_{\phi}$ is an algebra isomorphism since $n_{\phi}$ is abelian.

Since $\lambda\left(s\left(n_{\phi}\right)^{m}\right)=r_{\phi}^{m}$, Theorem 4.6 shows that the elements $q_{\phi}$ and $y$ in Case 3 and $q_{\phi}$ and $q_{2 \phi}$ in Case 4 lie in $\pi_{\phi}^{m}$. Also, since $g^{2 \phi}$ is central in $n_{\phi}$, we see that $q_{\phi}$ commutes with $y$ in Case 3 and $q_{2 \phi}$ in Case 4.

Denote the usual filtration of the enveloping algebra $\pi_{\phi}$ by $\pi_{0} \subset \pi_{1} \subset \pi_{2} \subset \ldots$. so that $\pi_{0}=k \cdot 1$ and $\pi_{1}=k \cdot 1 \oplus n_{\phi}$, and for each $r \in \mathbf{Z}_{+}$let $\pi_{r}: \pi_{r} \rightarrow \pi_{r} / \pi_{r-1}$ be the canonical map. (Here we take $\pi_{-1}=0$.) We also have the usual grading $S\left(n_{\phi}\right)=\amalg_{r=0}^{\infty} S^{r}\left(n_{\phi}\right)$ of $S\left(n_{\phi}\right)$. For each $r \in Z_{+}$, let $\sigma_{r}: S^{r}\left(n_{\phi}\right) \rightarrow$ $\pi_{r} / \pi_{r-1}$ be the canonical map, so that $\sigma_{r}$ is a linear isomorphism by the Poincaré-Birkhoff-Witt theorem (see [2, Proposition 2.3.6]).

Now suppose that we are in Case 3. We claim that $q_{\phi}$ and $y$ are algebraically independent. In fact, if not, then for some $r \in \mathbf{Z}_{+}$, there is an equation

$$
\sum_{j=0}^{r} \sum_{i=0}^{[j / 2]} a_{i j} q_{\phi}^{i} y^{j-2 i}=0,
$$

where the $a_{i j} \in k$, and some $a_{i r} \neq 0(i=0, \ldots,[r / 2]) ;[\cdot]$ denotes the "greatest integer" function. Thus $\sum_{i=0}^{[r / 2]} a_{i r} q_{\phi}^{i} y^{r-2 i} \in \Re_{p-1}$, so that

$$
\pi_{r}\left(\sum_{i=0}^{[r / 2]} a_{i r} q_{\phi}^{i} y^{r-2 i}\right)=0
$$

Consider the element

$$
s=\sum_{i=0}^{[r / 2]} a_{i r}\left(\frac{2}{(\phi, \phi)} p_{\phi}\right)^{i} y^{r-2 i} \in S^{r}\left(n_{\phi}\right)_{\circ}
$$


Then

$$
\begin{aligned}
\sigma_{r}(s) & =\pi_{r}(\lambda(s))=\pi_{r}\left(\sum_{i=0}^{[r / 2]} a_{i r} \lambda\left(\frac{2}{(\phi, \phi)} p_{\phi}\right)^{i} \lambda(y)^{r-2 i}\right) \\
& =\pi_{r}\left(\sum_{i=0}^{[r / 2]} a_{i r} q_{\phi}^{i} y^{r-2 i}\right)=0,
\end{aligned}
$$

and so $s=0$. But this is a contradiction, since $p_{\phi}$ and $y$ are algebraically independent in $S\left(n_{\phi}\right)$, and the claim is established. A similar argument shows that in Case 4, $q_{\phi}$ and $q_{2 \phi}$ are algebraically independent.

All that remains is to show that $q_{\phi}$ and $y$ generate $r_{\phi}^{\mathrm{m}}$ in Case 3 and that $q_{\phi}$ and $q_{2 \phi}$ generate $r_{\phi}^{m}$ in Case 4. We shall carry out the argument only for Case 3; Case 4 is similar. Assume inductively that $q_{\phi}$ and $y$ generate $\pi_{\phi}^{m} \cap \pi_{r}$, where $r \in \mathbf{Z}_{+}$. (This is trivially true for $r=0$.). Now

$$
r_{t}=\lambda\left(\prod_{i=0}^{t} s^{i}\left(n_{\phi}\right)\right)
$$

for all $t \in \mathbf{Z}_{+}$. Let

$$
z \in r_{\phi}^{m} \cap r_{r+1}=\lambda\left(s\left(n_{\phi}\right)^{m} \cap \coprod_{i=0}^{r+1} s^{i}\left(n_{\phi}\right)\right) .
$$

Then $z$ is of the form

$$
z=\lambda\left(\sum_{j=0}^{r+1} \sum_{i=0}^{[j / 2]} a_{i j}\left(\frac{2}{(\phi, \phi)} p_{\phi}\right)^{i} y^{j-2 i}\right)
$$

$\left(a_{i j} \in k\right)$ by Theorem 4.6. But

$$
z-\sum_{j=0}^{r+1} \sum_{i=0}^{[j / 2]} a_{i j} q_{\phi}^{i} y^{j-2 i} \in r_{\phi}^{m} \cap r_{r},
$$

and so the induction hypothesis implies that $z$ can be expressed as a polynomial in $q_{\phi}$ and $y$. This completes the proof of Theorem 5.1. Q.E.D.

6. The case $2 a \notin \Sigma$. In this section, we compute certain commutators in the universal enveloping algebra $\varrho$ of $g$, and then we use these to determine certain conical vectors in the twisted induced g-modules $X^{\nu}$, where $\nu \in a^{*}$ (see $\$ 2$ ). Specifically, we prove our main results (Theorems 10.1 and $10.2)$ in the special case in which twice the relevant restrict ed root is not a restricted root (see Theorems 6.17 and 6.18 ). But the first part of the section, through Lemma 6.4, is valid in general, and this will be important in $\S 8$.

Maintain the hypotheses and notation of the last section. For conven- 
ience assume for awhile (through Corollary 6.10) that $k$ is algebraically closed.

Continue to fix $\phi \in \Sigma$, and choose $h_{\phi}, e_{\phi}, f_{\phi}$, and $u_{\phi}$ as in $\$ 2$. Applying the constructions of the beginnings of $\$ \$ 4$ and 5 to $-\phi$ in place of $\phi$, we have canonical elements $p_{-\phi} \in S^{2}\left(g^{-\phi}\right)^{\mathrm{m}}$ and $q_{-\phi}=2 \lambda\left(p_{-\phi}\right) /(\phi, \phi) \in \pi_{-\phi}^{m}$. Our goal now is to compute the commutator $\left[e_{\phi}, q_{-\phi}\right]$ in $\mathcal{G}_{\text {. }}$.

Since $B_{\theta}\left(e_{\phi}, e_{\phi}\right)=2 /(\phi, \phi)$, it is clear that $B_{\theta}\left(f_{\phi}, f_{\phi}\right)=2(\phi, \phi)$ also. Using the notation of the proof of Lemma 4.7, we recall from Lemma 4.9 that the decomposition $g_{\phi}=g_{0} \oplus g_{1} \oplus g_{2}$ is $B_{\theta}$-orthogonal, and hence so is the decomposition $g^{-\phi}=g_{1}^{-1} \oplus g_{2}^{-1}$. Set $f_{1}=f_{\phi}$. Since $B_{\theta}$ is nonsingular on $g^{-\phi}$ and $k$ is algebraically closed, we may complete $f_{1}$ to a $B_{\theta}$-orthogonal basis $\left\{f_{1}, \ldots, f_{n}\right\}$ of $g^{-\phi}$ such that $B_{\theta}\left(f_{i}, f_{i}\right)=2 /(\phi, \phi)$ for all $i=1, \ldots$, n. But since $f_{1} \in g_{1}^{-1}$, we may also assume that $f_{1}, \ldots, f_{r} \in g_{1}^{-1}$ and that $f_{r+1}, \ldots, f_{n} \in g_{2}^{-1}$. Here $n=\operatorname{dim} g^{\phi}=\operatorname{dim} g^{-\phi}$ and $r=\operatorname{dim} g_{1}^{-1}$. Note that $\operatorname{dim} g^{2 \phi}=\operatorname{dim} g_{2}^{-1}=n-r$, and hence that $g^{2 \phi} \neq 0$ if and only if $r<n$.

The canonical element $p_{-\phi} \in S^{2}\left(g^{-\phi}\right)$ is equal to the sum of the squares of the elements of any $B_{\theta^{-o r t h o n o m a l ~ b a s i s ~ o f ~}} \mathrm{~g}^{-\phi}$, and so

$$
p_{-\phi}=\frac{(\phi, \phi)}{2} \sum_{i=1}^{n} f_{i}^{2}
$$

Thus

$$
q_{-\phi}=\frac{2}{(\phi, \phi)} \lambda\left(p_{-\phi}\right)=\sum_{i=1}^{n} f_{i}^{2} \in \Re_{-\phi} .
$$

To compute $\left[e_{\phi}, q_{-\phi}\right]$, we first note that

$$
\begin{aligned}
{\left[e_{\phi}, q_{-\phi}\right] } & =\sum_{i=1}^{n}\left[e_{\phi}, f_{i}^{2}\right]=\sum_{i=1}^{n}\left(\left[e_{\phi}, f_{i}\right] f_{i}+f_{i}\left[e_{\phi}, f_{i}\right]\right) \\
& =\sum_{i=1}^{n}\left(\left[\left[e_{\phi}, f_{i}\right], f_{i}\right]+2 f_{i}\left[e_{\phi}, f_{i}\right]\right) \\
& =\sum_{i=1}^{n}\left(\left[f_{i},\left[f_{i}, e_{\phi}\right]\right]+2 f_{i}\left[e_{\phi}, f_{i}\right]\right) .
\end{aligned}
$$

Lemma 6.1. $\left[f_{1},\left[f_{1}, e_{\phi}\right]\right]=-2 f_{1}$.

Proof. This follows immediately from the bracket relations for $h_{\phi}, e_{\phi}$ and $f_{1}=f_{\phi}$. Q.E.D.

Lemma 6.2. For all $i=2, \ldots, n,\left[e_{\phi}, f_{i}\right] \in \mathrm{m}$. 
Proof. Apply Lemma 4.10. Q.E.D.

Lemma 6.3. For all $i=2, \ldots,, r,\left[f_{i},\left[f_{i}, e_{\phi}\right]\right]=2 f_{1}$, and for all $i=r+$ $1, \ldots, n,\left[f_{i},\left[f_{i}, e_{\phi}\right]\right]=6 f_{1}$.

Proof. Let $i=2, \ldots, n$. Then

$$
\left[e_{\phi},\left[f_{i},\left[f_{i}, e_{\phi}\right]\right]\right]=-\left[e_{\phi},\left[f_{i},\left[e_{\phi}, f_{i}\right]\right]\right]=-\left[f_{i},\left[e_{\phi},\left[e_{\phi}, f_{i}\right]\right]\right] .
$$

But $\left[e_{\phi}, f_{i}\right] \in \mathfrak{m}$ (Lemma 6.2), so that

$$
\theta\left[e_{\phi},\left[e_{\phi}, f_{i}\right]\right]=\left[\theta e_{\phi},\left[e_{\phi}, f_{i}\right]\right]=-\left[f_{1},\left[e_{\phi}, f_{i}\right]\right] .
$$

Now we can apply the standard representation theory of the three-dimensional simple Lie algebra $u_{\phi}$. If $2 \leq i \leq r$, then $f_{i} \in g_{1}^{-1}$, and so $\left[f_{1},\left[e_{\phi}, f_{i}\right]\right]=2 f_{i}$, and if $r+1 \leq i \leq n$, then $f_{i} \in g_{2}^{-1}$, and so $\left[f_{1},\left[e_{\phi}, f_{i}\right]\right]=6 f_{i}$. Hence $\left[e_{\phi},\left[e_{\phi}, f_{i}\right]\right]=-2 \theta f_{i}$ or $-6 \theta f_{i}$, respectively, and so

$$
\left[e_{\phi},\left[f_{i},\left[f_{i}, e_{\phi}\right]\right]\right]=2\left[f_{i}, \theta f_{i}\right] \text { or } \sigma\left[f_{i}, \theta f_{i}\right] \text {, }
$$

respectively. But $\left[f_{i}, \theta f_{i}\right]=-B_{\theta}\left(f_{i}, f_{i}\right) x_{-\phi}($ see $\$ 2)$, and this is just $h_{\phi}$. Thus

$$
\left[e_{\phi},\left[f_{i},\left[f_{i}, e_{\phi}\right]\right]\right]=2 h_{\phi} \text { or } 6 h_{\phi} \text {, }
$$

respectively. But $\left[f_{i},\left[f_{i}, e_{\phi}\right]\right] \in g^{-\phi}$, and so has eigenvalue -2 for ad $h_{\phi}$. Since $\left[e_{\phi},\left[f_{i},\left[f_{i}, e_{\phi}\right]\right]\right]$ is a multiple of $h_{\phi}$, the representation theory of $\mathfrak{u}_{\phi}$ implies that $\left[f_{i},\left[f_{i}, e_{\phi}\right]\right]$ must be a multiple of $f_{1}$. Since $\left[e_{\phi}, f_{1}\right]=h_{\phi}$, the multiple is determined and the lemma follows. Q.E.D.

In view of these lemmas, we have

$$
\begin{aligned}
{\left[e_{\phi}, q_{-\phi}\right] } & =-2 f_{1}+2 f_{1} h_{\phi}+2(r-1) f_{1}+6(n-r) f_{1}+2 \sum_{i=2}^{n} f_{i}\left[e_{\phi}, f_{i}\right] \\
& =2\left((3 n-2 r-2) f_{1}+f_{1} h_{\phi}+\sum_{i=2}^{n} f_{i}\left[e_{\phi}, f_{i}\right]\right) .
\end{aligned}
$$

Let

$$
\rho_{\phi}=1 / 2\left(\left(\operatorname{dim} g^{\phi}\right) \phi+\left(\operatorname{dim} g^{2 \phi}\right)(2 \phi)\right) \epsilon a^{*} .
$$

Then $\rho_{\phi}=1 / 2(n+2(n-r)) \phi=1 / 2(3 n-2 r) \phi$, and so $\rho_{\phi}\left(h_{\phi}\right)=3 n-2 r$. The conclusion is:

Lemma 6.4. Define $\rho_{\phi}$ as in (1). Then

$$
\left[e_{\phi}, q_{-\phi}\right]=2\left(\left(\rho_{\phi}-\phi\right)\left(b_{\phi}\right) f_{\phi}+f_{\phi} h_{\phi}+\sum_{i=2}^{n} f_{i}\left[e_{\phi}, f_{i}\right]\right) \text {. }
$$


We could now use the derivation law to write down an expression for [ $e_{\phi}, q_{-\phi}^{d}$ ], for all $d \in \mathrm{Z}_{+}$. In order to simplify matters, however, we shall assume at this point that $g^{2 \phi}=0$, which implies that $g^{-\phi}$ is an abelian Lie subalgebra of $\mathrm{g}$. The much subtler general situation is deferred to subsequent sections.

Lemma 6.5. Suppose $2 \phi \notin \Sigma$. For all $d \in \mathbf{Z}_{+}$,

$$
\left[e_{\phi}, q_{-\phi}^{d}\right]=2 d q_{-\phi}^{d-1}\left(f_{\phi}\left(h_{\phi}+\left(\rho_{\phi}-d \phi\right)\left(h_{\phi}\right)\right)+\sum_{i=2}^{n} f_{i}\left[e_{\phi}, f_{i}\right]\right) \text {. }
$$

Proof. From Lemmas 6.4 and 6.2 and the commutativity of $\mathrm{g}^{-\phi}$, we get

$$
\begin{aligned}
{\left[e_{\phi}, q_{-\phi}^{d}\right]=} & \sum_{j=1}^{d} q_{-\phi}^{d-j}\left[e_{\phi}, q_{-\phi}\right] q_{-\phi}^{j-1} \\
= & 2 d q_{-\phi}^{d-1}\left(\left(\rho_{\phi}-\phi\right)\left(h_{\phi}\right) f_{\phi}+\sum_{i=2}^{n} f_{i}\left[e_{\phi}, f_{i}\right]\right) \\
& +2 f_{\phi} \sum_{j=1}^{d} q_{-\phi}^{d-j} h_{\phi} q_{-\phi}^{j-1} .
\end{aligned}
$$

But $q_{-\phi}$ is clearly a restricted weight vector for the action of $a$ on $\oint$ with restricted weight $-2 \phi$, and so

$$
\begin{aligned}
h_{\phi} q_{-\phi}^{j-1} & =\left[h_{\phi}, q_{-\phi}^{j-1}\right]+q_{-\phi}^{j-1} h_{\phi} \\
& =-2(j-1) \phi\left(h_{\phi}\right) q_{-\phi}^{j-1}+q_{-\phi}^{j-1} h_{\phi}=q_{-\phi}^{j-1}\left(-4(j-1)+h_{\phi}\right) .
\end{aligned}
$$

Hence

$$
\sum_{i=1}^{d} q_{-\phi}^{d-j} h_{\phi} q_{-\phi}^{j-1}=\sum_{j=1}^{d} q_{-\phi}^{d-1}\left(-4(j-1)+h_{\phi}\right)=q_{-\phi}^{d-1}\left(d h_{\phi}-2 d(d-1)\right),
$$

and so

$$
\begin{aligned}
{\left[e_{\phi}, q_{-\phi}^{d}\right]=} & \left.2 d q_{-\phi}^{d-1}\left(\phi_{d}-\phi\right)\left(h_{\phi}\right) f_{\phi}+\sum_{i=2}^{n} f_{i}\left[e_{\phi}, f_{i}\right]\right) \\
& +2 d q_{-\phi}^{d-1} f_{\phi} h_{\phi}-4 d q_{-\phi}^{d-1} f_{\phi}(d-1) \\
= & 2 d q_{-\phi}^{d-1}\left(f_{\phi}\left(\rho_{\phi}\left(h_{\phi}\right)-2-2(d-1)+h_{\phi}\right)+\sum_{i=2}^{n} f_{i}\left[e_{\phi}, f_{i}\right)\right. \\
= & 2 d q_{-\phi}^{d-1}\left(f_{\phi}\left(h_{\phi}+\left(\rho_{\phi}-d \phi\right)\left(h_{\phi}\right)\right)+\sum_{i=2}^{n} f_{i}\left[e_{\phi}, f_{i}\right]\right) \cdot \text { Q.E.D. }
\end{aligned}
$$


The following result is now immediate:

Corollary 6.6. Suppose $\phi \in \Sigma+$ and $2 \phi \notin \Sigma$. Let $X$ be a g-module and $x \in X$ a conical restricted weight vector with restricted weight $\mu \in a^{*}$. Then for all $d \in \mathbf{Z}_{+}$,

$$
e_{\phi} \cdot\left(q_{-\phi}^{d} \cdot x\right)=2 d\left(\left(\mu+\rho_{\phi}-d \phi\right)\left(h_{\phi}\right)\right) f_{\phi} q_{-\phi}^{d-1} \cdot x_{0}
$$

If $\operatorname{dim} g^{\phi}=1$ (in which case $g^{ \pm 2 \phi}=0$ automatically), we also have the following lemma and corollary:

Lemma 6.7. Suppose $\operatorname{dim} g^{\phi}=1$. Then for all $d \in \mathbf{Z}_{+}$,

$$
\left[e_{\phi}, f_{\phi}^{d}\right]=d f_{\phi}^{d-1}\left(h_{\phi}+\left(\rho_{\phi}-d \phi / 2\right)\left(h_{\phi}\right)\right) \text {. }
$$

Proof. Since $\left[e_{\phi}, f_{\phi}\right]=h_{\phi}$, we have

$$
\left[e_{\phi}, f_{\phi}^{d}\right]=\sum_{j=1}^{d} f_{\phi}^{d-j} h_{\phi} f_{\phi}^{j-1} .
$$

But

$$
h_{\phi} f_{\phi}^{j-1}=\left[h_{\phi}, f_{\phi}^{j-1}\right]+f_{\phi}^{j-1} h_{\phi}=f_{\phi}^{j-1}\left(-2(j-1)+h_{\phi}\right),
$$

so that

$$
\left[e_{\phi}, f_{\phi}^{d}\right]=f_{\phi}^{d-1}\left(d h_{\phi}-d(d-1)\right)=d f_{\phi}^{d-1}\left(h_{\phi}+\left(\rho_{\phi}-d \phi / 2\right)\left(h_{\phi}\right)\right),
$$

since $\rho_{\phi}\left(h_{\phi}\right)=1 / 2 \phi\left(h_{\phi}\right)=1$. Q.E.D.

Corollary 6.8. Suppose $\phi \in \Sigma_{+}$and $\operatorname{dim} g^{\phi}=1$. Let $X$ be a g-module and $x \in X$ an n-invariant restricted weight vector with restricted weight $\mu \in a^{*}$. Then for all $d \in \mathbf{Z}_{+}$,

$$
e_{\phi} \cdot\left(f_{\phi}^{d} \cdot x\right)=d\left(\left(\mu+\rho_{\phi}-d \phi / 2\right)\left(h_{\phi}\right)\right) f_{\phi}^{d-1} \cdot x
$$

Corollaries 6.6 and 6.8 imply the following two results. These have the benefit of being true even if $k$ is not algebraically closed, as the field extension technique shows; we also use the fact that the $B_{\theta^{-n} \text { nonisotropic vectors }}$ in $g^{\phi}$ span $g^{\phi}$.

Corollary 6.9. Suppose $\phi \in \Sigma_{+}$and $2 \phi \notin \Sigma_{+}$. Let $X$ be a g-module and $x \in X$ a conical restricted weight vector with restricted weight $\mu \in a^{*}$. Then for all $e_{0} \in g^{\phi}$ and $d \in \mathbf{Z}_{+}$, 


$$
e_{0} \cdot\left(q_{-\phi}^{d} \cdot x\right)=-2 d\left(\left(\mu+\rho_{\phi}-d \phi\right)\left(h_{\phi}\right)\right)\left(\theta e_{0}\right) q_{-\phi}^{d-1} \cdot x .
$$

Corollary 6.10. Suppose $\phi \in \Sigma_{+}$and $\operatorname{dim} g^{\phi}=1$. Let $X$ be a g-module and $x \in X$ an n-invariant restricted weight vector with restricted weight $\mu \in a^{*}$. Then for all $e_{0} \in g^{\phi}$ and $d \in \mathbf{Z}_{+}$,

$$
e_{0} \cdot\left(\left(\theta e_{0}\right)^{d} \cdot x\right)=-1 / 2 B_{\theta}\left(e_{0}, e_{0}\right)(\phi, \phi) d\left(\left(\mu+\rho_{\phi}-d \phi / 2\right)\left(h_{\phi}\right)\right)\left(\theta e_{0}\right)^{d-1} \cdot x_{.}
$$

Assume for the rest of this section that $k$ is an arbitrary field of characteristic zero-not necessarily algebraically closed. We are now ready to prove the following basic result:

Lemma 6.11. Suppose $\phi \in \Sigma_{+}$and $2 \phi \notin \Sigma_{+}$. Let $\nu \in a^{*}$, and let $x_{0}$ be the canonical generator of the twisted induced g-module $X^{\nu}=V^{\nu-\rho}$ (see \$2). Set $Y=\left(\pi_{-\phi} \cdot x_{0}\right)^{m \oplus n_{\phi}}$ (see $\oint_{5}$ for the definitions of $\pi_{-\phi}$ and $n_{\phi}$ ), and de. fine $h_{\phi}^{\prime} \in a$ to be, $h_{\phi}$ if $\operatorname{dim} g^{\phi}>1$ and $2 h_{\phi}$ if $\operatorname{dim} g^{\phi}=1$. If $\left(\nu-\rho+\rho_{\phi}\right)\left(h_{\phi}^{\prime}\right)$ is not a positive even integer, then $Y$ is the span of $x_{0}$. Suppose $\left(\nu-\rho+\rho_{\phi}\right)\left(h_{\phi}^{\prime}\right)=2 l, l$ a positive integer. Then $Y$ is two-dimensional, with basis $\left\{x_{0}, f^{l} \cdot x_{0}\right\}$, where $f=q_{-\phi}$ if $\operatorname{dim} g^{\phi}>1$ and $f$ is a nonzero element of $g^{-\phi}$ if $\operatorname{dim} g^{\phi}=1$. In this case, $f^{l} \cdot x_{0}$ is a restricted weight vector in $X^{\nu}$ with restricted weight $s_{\phi}\left(\nu-\rho+\rho_{\phi}\right)-\rho_{\phi}$ (recall from $\$ 2$ that $s_{\phi}$ is the Weyl reflection with respect to $\phi)$.

Proof. Since the map $\omega: \Re-\rightarrow X^{\nu}$ which takes $y \in \Re-$ to $y \cdot x_{0}$ is an m-module isomorphism (see $\$ 2$ ), we see that

$$
\left(r_{-\phi} \cdot x_{0}\right)^{m}=\left(\omega\left(r_{-\phi}\right)\right)^{m}=r_{-\phi}^{m} \cdot x_{0} .
$$

But by Theorem 5.1 (Cases 1 and 2), $\pi_{-\phi}^{\mathrm{m}}$ is the polynomial algebra $k[f]$, where $f$ is as in the statement of the lemma. Hence

$$
\left(\pi_{-\phi} \cdot x_{0}\right)^{m}=k[f] \cdot x_{0} .
$$

Let $u \in k[f]$, so that $u=\Sigma_{d=0}^{\infty} a_{d} f^{d}\left(a_{d} \in k\right.$, and only finitely many $\left.a_{d} \neq 0\right)$, and let $e_{0}$ be a $B$-nonisotropic vector in $g^{\phi}$; if $\operatorname{dim} g^{\phi}=1$, take $e_{0}=\theta f$. Suppose $\operatorname{dim} g^{\phi}>1$. Then by Corollary 6.9,

$$
e_{0} \cdot\left(u \cdot x_{0}\right)=-2 \sum_{d=0}^{\infty} a_{d} d\left(\left(\nu-\rho+\rho_{\phi}-d \phi\right)\left(h_{\phi}\right)\right)\left(\theta e_{0}\right) q_{-\phi}^{d-1} \cdot x_{0},
$$

and this expression is zero if and only if $a_{d} d\left(\left(\nu-\rho+\rho_{\phi}-d \phi\right)\left(h_{\phi}\right)\right)=0$ for all $d$. But this is the case if and only if $a_{d}=0$ for all $d>0$ such that $\left(\nu-\rho+\rho_{\phi}\right)\left(h_{\phi}\right) \neq 2 d$. The lemma for $\operatorname{dim} g^{\phi}>1$ now follows from Corollary 4.3; the last assertion of the lemma is clear since $q_{-\phi}^{l} \cdot x_{0}$ has restricted 
weight $\nu-\rho-2 l \phi$, and

$$
s_{\phi}\left(\nu-\rho+\rho_{\phi}\right)=\left(\nu-\rho+\rho_{\phi}\right)-\left(\nu-\rho+\rho_{\phi}\right)\left(h_{\phi}\right) \phi=\nu-\rho+\rho_{\phi}-2 l \phi .
$$

The case $\operatorname{dim} g^{\phi}=1$ is similar, using Corollary 6.10. Q.E.D.

Remark. Note that Lemma 6.11 holds when $\phi$ is not necessarily a simple restricted root, and even when $1 / 2 \phi$ is a restricted root.

The situation in Lemma 6.11 simplifies nicely when $\operatorname{dim} a=1$; the next result is an immediate consequence of the lemma:

Theorem 6.12. Suppose $\operatorname{dim} a=1$ and $\phi \in \Sigma_{+}$is the only positive root. Let $\nu \in a^{*}$. Then the conical space $Y$ of the twisted induced g-module $X^{\nu}$ is either one-or two-dimensional. Define $h_{\phi}^{\prime} \in$ a to be $h_{\phi}$ if $\operatorname{dim} g^{\phi}>1$ and $2 h_{\phi}$ if $\operatorname{dim} g^{\phi}=1$, and let $x_{0}$ be the canonical generator of $X^{\nu}$. If $\nu\left(h_{\phi}^{\prime}\right)$ is not a positive even integer, then $Y$ is the span of $x_{0}$. Suppose $\nu\left(b_{\phi}^{\prime}\right)=2 l$, $l$ a positive integer. Then $\operatorname{dim} Y=2$, and $Y$ has basis $\left\{x_{0}, f^{l} \cdot x_{0}\right\}$, where $f=q_{-\phi}$ if $\operatorname{dim} g^{\phi}>1$ and $f$ is a nonzero element of $g^{-\phi}$ if $\operatorname{dim} g^{\phi}=1$. In this case, $f^{l} \cdot x_{0}$ is a restricted weight vector in $X^{\nu}$ with restricted weight $s_{\phi} \nu-\rho$.

Lemma 6.11 also gives some interesting information about the conical space of $X^{\nu}$ even when $\operatorname{dim} a$ is arbitrary. To see this, we need some general facts.

Lemma 6.13. Let $\Pi \subset \Sigma_{+}$be the set of simple restricted roots. Then the subalgebra $n$ of $g$ is generated by the subspaces $g^{a}$ as a ranges through II.

Proof. We may, and do, assume that $k$ is algebraically closed. For all $\psi \in \Sigma$, define the order $o(\psi)$ of $\psi$ to be the integer $\Sigma n_{a}(\alpha \in \Pi)$, where the integers $n_{\alpha}$ are defined by the condition $\psi=\Sigma n_{a} \alpha(\alpha \in \Pi)$. Then $\psi \in \Sigma+$ if and only if $o(\psi)>0$, and $\psi \in \Pi$ if and only if $o(\psi)=1$. We shall show by induction on $d \psi\left(\psi \in \Sigma_{+}\right)$that $g^{\psi}$ lies in the space generated by the $g^{a}$ $(a \in \Pi)$. This is clearly true if $o(\psi)=1$, so assume it is true for $\sigma(\psi)=m$ $(m \geq 1)$, and let $\psi^{\prime} \in \Sigma_{+}$have order $m+1$. Then the standard theory of root systems shows that there exists $\alpha \in \Pi$ such that the scalar product $\left(\psi^{\prime}, \alpha\right)$ $>0$, and hence $\psi=\psi^{\prime}-\alpha$ is a positive restricted root of order $m$. Define the subspace $V$ of $g$ by $V=\amalg_{j=-\infty}^{\infty} g^{\psi+n a}$, and construct as in $\$ 2$ (taking $\alpha$ for $\phi$ ) a subalgebra $u_{\alpha}$ of $g$ spanned by $h_{a}, e_{a}$ and $f_{a}$. Then $V$ is a $u_{\alpha}$ submodule of $g$, and ad $h_{a}$ has eigenvalue $\psi\left(h_{\alpha}\right)+2 n$ on the subspace $g^{\psi+n a}$ of $V$; in particular, $g^{\psi+n a}$ is exactly the $\left(\psi\left(h_{\alpha}\right)+2 n\right)$-eigenspace for ad $h_{a}$ in $V$. But 


$$
\psi\left(h_{a}\right)+2=(\psi+\alpha)\left(b_{\alpha}\right)=\psi^{\prime}\left(h_{\alpha}\right)>0,
$$

and so the integer $\psi\left(h_{\alpha}\right) \geq-1$. Hence ad $b_{a}$ has eigenvalue $\geq-1$ on $g^{\psi}$, and so by the representation theory of the three-dimensional simple Lie algebra $\mathfrak{u}_{a}$, we see that $\left[e_{\alpha}, g^{\psi}\right]=g^{\psi+a}=g^{\psi^{\prime}}$, and so $\left[g^{a}, g^{\psi}\right]=g^{\psi^{\prime}}$. In view of the induction hypothesis, we are finished. Q.E.D.

Remark. The above proof is of course similar to the proof of Lemma 4.2.

Lemma 6.14. Let $a \in \Pi$ (see Lemma 6.13). Then $s_{a} \rho-\rho=s_{a} \rho_{a}-\rho_{a}$, and $\rho\left(h_{\alpha}\right)=\rho_{\alpha}\left(h_{\alpha}\right)$.

Proof. The first assertion is proved in [7(b), Lemma 4.16]. It follows that

$$
\rho-\rho_{\alpha}=s_{\alpha}\left(\rho-\rho_{\alpha}\right)=\left(\rho-\rho_{\alpha}\right)-\left(\rho-\rho_{\alpha}\right)\left(h_{\alpha}\right) \alpha_{,}
$$

and so $\left(\rho-\rho_{a}\right)\left(h_{\alpha}\right)=0$. Q.E. D.

Lemma 6.15. $\mathrm{r}^{-}$is a direct sum of restricted weight spaces (with re.

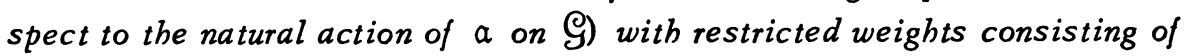
those elements of $a^{*}$ of the form $-\Sigma n_{\beta} \beta$, where $\beta$ ranges through $\Pi$ and $n_{\beta} \in \mathbf{Z}_{+}$. Let $\alpha \in \Pi$, and suppose $y \in \Re(-$ is a restricted weight vector with restricted weight of the form $c \alpha(c \in k)$. Then $y \in \Re_{-}$and $c \in-Z_{+}$.

Proof. Let $\Sigma_{+}^{1}=\left\{\psi \in \Sigma_{+} \mid 1 / 2 \psi \notin \Sigma_{+}\right\}$. Then $n^{-}=\amalg n_{-\psi}$ as $\psi$ ranges through $\Sigma_{+}^{1}$. Let $\psi_{1}, \psi_{2}, \ldots, \psi_{p}$ be the elements of $\Sigma_{+}^{-}$. Then the multiplication map in $\mathcal{G}$ induces a linear isomorphism

$$
r_{-} \simeq \pi_{-\psi_{1}} \otimes r_{-\psi_{2}} \otimes \cdots \otimes r_{-\psi_{p}}
$$

The lemma now follows easily. Q.E.D.

Lemma 6.16. Let $a \in \Pi, \nu \in a^{*}$ and $x_{0}$ the canonical generator of the twisted induced module $X^{\nu}$. The sum of the restricted weight spaces of $X^{\nu}$ with restricted weights of the form $\nu-\rho+c \alpha(c \in k)$ is exactly $\pi_{-a} \cdot x_{0}$.

Proof. This is clear from Lemma 6.15 and the fact that the linear isomorphism $\omega: \Re-\rightarrow X^{\nu}$ which takes $y$ to $y \cdot x_{0}$ raises restricted weights by $\nu-\rho$; i.e., if $y \in \Re-$ is a restricted weight vector with restricted weight $\mu \in a^{*}$, then $\omega(y)$ is a restricted weight vector with restricted weight $\nu-\rho$ $+\mu$. Q.E. D.

We now have the following generalization of Theorem 6.12:

Theorem 6.17. Let $a$ be a simple restricted root, and suppose $2 \alpha \notin \Sigma$. Let $\nu \in a^{*}$, and let $Y$ be the subspace of the twisted induced g-module $X^{\nu}$ spanned by the conical restricted weight vectors with restricted weights of 
the form $\nu-\rho+c a(c \in k)$. Then $Y$ is either one-or two-dimensional. Define $h_{\alpha}^{\prime} \in$ a to be $h_{\alpha}$ if $\operatorname{dim} g^{a}>1$ and $2 h_{\alpha}$ if $\operatorname{dim} g^{a}=1$, and let $x_{0}$ be the canonical generator of $X^{\nu}$. If $\nu\left(h_{a}^{\prime}\right)$ is not a positive even integer, then $Y$ is the span of $x_{0}$. Suppose $\nu\left(h_{\alpha}^{\prime}\right)=2 l, l$ a positive integer. Then $\operatorname{dim} Y=2$, and $Y$ has basis $\left\{x_{0}, f^{l} \cdot x_{0}\right\}$, where $f=q_{-} a$ if $\operatorname{dim} g^{\alpha}>1$ and $f$ is a non. zero element of $g^{-\alpha}$ if $\operatorname{dim} g^{\alpha}=1$. In this case, $f^{l} \cdot x_{0}$ is a restricted weight vector in $X^{\nu}$ with restricted weight $s_{\alpha} \nu-\rho$.

Proof. Since the conical space of $X^{\nu}$ is clearly a-stable and hence the direct sum of its intersections with the restricted weight spaces of $X^{\nu}, Y=$ $\left(\pi_{-\alpha} \cdot x_{0}\right)^{\mathrm{m} \oplus \mathrm{n}}$ by Lemma 6.16. Let $y \in\left(\boldsymbol{r}_{-\alpha} \cdot x_{0}\right)^{\mathrm{m} \oplus \mathfrak{n}_{\alpha}}$, so that $y=u \cdot x_{0}$, where $u \in \pi_{-}$. Let $\beta$ be a simple restricted root not equal to $a$. Then $\beta-\alpha$ is not a restricted root and is not zero, so that $\left[g^{\beta}, n_{-\alpha}\right]=\left[g^{\beta}, g^{-\alpha}\right]$ $=0$. Hence $\left[\mathrm{g}^{\beta}, u\right]=0$ in $\mathcal{G}$, and so

$$
g^{\beta} \cdot\left(u \cdot x_{0}\right)=u \cdot\left(g^{\beta} \cdot x_{0}\right)=0 .
$$

Lemma 6.13 now shows that $y \in Y$. Thus $Y=\left(\pi_{-a} \cdot x_{0}\right)^{m \oplus n_{a}}$, and the theorem now follows from Lemmas 6.11 and 6.14. Q.E.D.

Remark. In the notation of Theorem 6.17 , the assertion that $\nu\left(h_{\alpha}^{\prime}\right)$ be a nonnegative even integer (possibly zero) is equivalent to the existence in $X^{\nu}$ of an m-invariant restricted weight vector with restricted weight $s_{\alpha} \nu-\rho=$ $\nu-\rho-\nu\left(h_{\alpha}\right) \alpha$ (use Lemma 6.16). In this case, the m-invariant restricted weight vectors with restricted weight $s_{\alpha} \nu-\rho$ span a one-dimensional space and are conical vectors. Note also that if $\nu \in a^{*}$ is arbitrary and if $f$ and $x_{0}$ are defined as in Theorem 6.17, then $f^{m} \cdot x_{0}$ ( $m$ a positive integer) is $n$ invariant if and only if its restricted weight is $s_{\alpha} \nu-\rho$.

We can reformulate our conclusions as follows:

Theorem 6.18. Let a be a simple restricted root such that $2 a \notin \Sigma$. Let $\mu, \nu \in a^{*}$, and suppose that $\mu-\nu$ is of the form ca $(c \in k)$. (If $\operatorname{dim} a=1$, then this is automatic.) Then $\operatorname{Hom}_{\mathrm{g}}\left(X^{\mu}, X^{\nu}\right)$ is at most one-dimensional, and $\operatorname{dim} \operatorname{Hom}_{g}\left(X^{\mu}, X^{\nu}\right)=1$ if and only if either $\mu=\nu$, or else $\mu=s_{\alpha} \nu$ and $\nu\left(h_{\alpha}^{\prime}\right)$ is a nonnegative even integer, where $h_{\alpha}^{\prime}=h_{\alpha}$ if $\operatorname{dim} g^{a}>1$ and $h_{\alpha}^{\prime}=$ $2 h_{\alpha}$ if $\operatorname{dim} g^{\alpha}=1$. Also, $\operatorname{dim} \operatorname{Hom}_{g}\left(x^{\mu}, X^{\nu}\right)=1$ if and only if $X^{\mu}$ is isomorphic to a g-submodule of $X^{\nu}$.

Proof. Recall from $\$ 2$ that $\operatorname{Hom}_{g}\left(X^{\mu}, X^{\nu}\right)$ is isomorphic to the intersection $Z$ of the conical space of $X^{\nu}$ with the restricted weight space for $\mu-\rho$. If $\mu=\nu$, then clearly $\operatorname{dim} Z=1$. Suppose $\mu=s_{a} \nu$ and $\nu\left(h_{a}^{\prime}\right)$ is a nonnegative even integer. Then the above remark implies that $\operatorname{dim} Z=1$. Conversely, suppose $Z \neq 0$, so that $X^{\nu}$ contains a conical restricted weight vector $x$ 
with restricted weight $\mu-\rho$. Since $\mu=\nu+c \alpha, \mu-\rho=\nu-\rho+c \alpha$, and so $x \in Y$, in the notation of Theorem 6.17. If $\mu \neq \nu$, then $x$ is not a multiple of $x_{0}$ (again in the notation of Theorem 6.17), so that $\nu\left(h_{\alpha}^{\prime}\right)$ is a positive even integer and $\mu-\rho=s_{a} \nu-\rho$, i.e., $\mu=s_{a} \nu$, by Theorem 6.17. The last assertion of the theorem follows from the fact that any nonzero $g$-module map from $X^{\mu}$ into $X^{\nu}$ is injective (see $\$ 2$ ). Q. E.D.

7. The fundamental commutation relation in $\pi_{-\phi}$. We shall continue to use the notation of $\$ 6$, with $k$ algebraically closed. But in this section, we explicitly assume that $g^{2 \phi} \neq 0$, i.e., that $2 \phi \in \Sigma$. We have the canonical elements $p_{-2 \phi} \in S^{2}\left(g^{-2 \phi}\right)^{m}$ and $q_{-2 \phi}=\lambda\left(p_{-2 \phi}\right) / 2(\phi, \phi) \in \pi_{-\phi}^{\mathfrak{m}}$ (see $\left.\$ 5\right)$.

It is clearly important to compute the commutator $\left[e_{\phi}, q_{-2 \phi}\right]$ in $\mathcal{G}$. This will easily turn out to be essentially $\left[f_{\phi}, q_{-\phi}\right]$, and we have to know to what extent this element commutes with $q_{-} \phi$. In particular, we want to compute $\left[\left[f_{\phi}, q_{-\phi}\right], q_{-\phi}\right]$. Lemma 6.4 also points out the importance of this commutator, since we need it in principle to simplify the commutator $\left[e_{\phi}, q_{-\phi}^{d}\right]$. It will turn out that $\left[\left[f_{\phi}, q_{-\phi}\right], q_{-\phi}\right]$ is essentially $f_{\phi} q_{-2 \phi}$, and this is what we call the fundamental commutation relation in $\pi_{-\phi}$, the main result of this section. Because of this, we know how to compute the further commutators $\left[\cdots\left[\left[f_{\phi}, q_{-\phi}\right], q_{-\phi}\right] \cdots q_{-\phi}\right]$. The abstract algebraic setting in the next section will reveal a more precise reason for calling our relation "fundamental". The point will be that the fundamental relation and the trivial relation $f_{\phi} q_{-2 \phi}=q_{-2 \phi} f_{\phi}$ are in a sense all the relations involving $f_{\phi}, q_{-\phi}$ and $q_{-2 \phi}$

Lemma 7.1. The map ad $f_{\phi}: g_{2}^{-1} \rightarrow g_{2}^{-2}$ is an isometry from $4 B_{\theta} \mid g_{2}^{-1} \times$ $g_{2}^{-1}$ to $B_{\theta} \mid g_{2}^{-2} \times g_{2}^{-2}$.

Proof. Let $x, y \in g_{2}^{-1}$. Then

$$
\begin{aligned}
B_{\theta}\left(\left[f_{\phi}, x\right],\left[f_{\phi}, y\right]\right) & =-B\left(\left[f_{\phi}, x\right], \theta\left[f_{\phi}, y\right]\right)=B\left(\left[f_{\phi}, x\right],\left[e_{\phi}, \theta y\right]\right) \\
& =-B\left(\left[e_{\phi},\left[f_{\phi}, x\right]\right], \theta y\right)=-4 B(x, \theta y)
\end{aligned}
$$

(by Lemma 4.15)

$$
=4 B_{\theta}(x, y) \text {. Q.E.D. }
$$

Recall from $\$ 6$ the $B_{\theta^{-o r t h o g o n a l}}$ basis $\left\{f_{1}, \ldots, f_{n}\right\}$ of $g^{-\phi}$.

Lemma 7.2. We have

$$
q_{-2 \phi}=\frac{1}{16} \sum_{i=1}^{n}\left[f_{\phi}, f_{i}\right]^{2}=\frac{1}{16} \sum_{i=r+1}^{n}\left[f_{\phi}, f_{i}\right]^{2} .
$$

Proof. By Lemma 7.1, $\left\{\left[f_{\phi}, f_{r+1}\right], \ldots,\left[f_{\phi}, f_{n}\right]\right\}$ is a $B_{\theta}$-orthogonal 
basis of $g_{2}^{-2}=g^{-2 \phi}$ such that each $B_{\theta}\left(\left[f_{\phi}, f_{i}\right],\left[f_{\phi}, f_{i}\right]\right)=8 /(\phi, \phi)$. Since $q_{-2 \phi}$ is $1 / 2(\phi, \phi)$ times the sum of the squares of the elements of any $B_{\theta}$ orthonormal basis of $g^{-2 \phi}$, we must have $q_{-2 \phi}=(1 / 16) \sum_{i=r+1}^{n}\left[f_{\phi}, f_{i}\right]^{2}$. But $\left[f_{\phi}, f_{j}\right]=0$ if $j=1, \ldots, r$ and so the lemma follows. Q.E.D.

Lemma 7.3. We have

$$
\begin{aligned}
{\left[e_{\phi}, q_{-2 \phi}\right] } & =\frac{1}{4}\left[f_{\phi}, q_{-\phi}\right]=\frac{1}{2} \sum_{i=1}^{n} f_{i}\left[f_{\phi}, f_{i}\right] \\
& =\frac{1}{2} \sum_{i=r+1}^{n} f_{i}\left[f_{\phi}, f_{i}\right] .
\end{aligned}
$$

Proof. By Lemma 7.2,

$$
\begin{aligned}
{\left[e_{\phi}, q_{-2 \phi}\right]=} & \frac{1}{16} \sum_{i=r+1}^{n}\left[e_{\phi},\left[f_{\phi}, f_{i}\right]^{2]}\right. \\
= & \frac{1}{16} \sum_{i=r+1}^{n}\left(\left[e_{\phi},\left[f_{\phi}, f_{i}\right]\right]\left[f_{\phi}, f_{i}\right]\right. \\
& \left.\quad+\left[f_{\phi}, f_{i}\right]\left[e_{\phi},\left[f_{\phi}, f_{i}\right]\right]\right) \\
= & \frac{1}{4} \sum_{i=r+1}^{n}\left(f_{i}\left[f_{\phi}, f_{i}\right]+\left[f_{\phi}, f_{i}\right] f_{i}\right)
\end{aligned}
$$

(by Lemma 4.15)

$$
=\frac{1}{2} \sum_{i=r+1}^{n} f_{i}\left[f_{\phi}, f_{i}\right]
$$

(since $\left[f_{\phi}, f_{i}\right] \in \mathrm{g}^{-2 \phi}$, which is central in $\pi_{-\phi}$ )

$$
=\frac{1}{2} \sum_{i=1}^{n} f_{i}\left[f_{\phi}, f_{i}\right]
$$

On the other hand, $q_{-\phi}=\sum_{i=1}^{n} f_{i}^{2}$, so that

$$
\begin{aligned}
{\left[f_{\phi}, q_{-\phi}\right] } & =\sum_{i=1}^{n}\left[f_{\phi}, f_{i}^{2}\right] \\
& =\sum_{i=1}^{n}\left(\left[f_{\phi}, f_{i}\right] f_{i}+f_{i}\left[f_{\phi}, f_{i}\right]\right) \\
& =2 \sum_{i=1}^{n} f_{i}\left[f_{\phi}, f_{i}\right] \text { Q. E. D. }
\end{aligned}
$$

Theorem 7.4. (The fundamental commutation relation in $\pi_{-} \phi^{\circ}$ ) We have

$$
\left[\left[f_{\phi}, q_{-\phi}\right], q_{-\phi}\right]=-64 f_{\phi} q_{-2 \phi} .
$$


More generally, suppose the field $k$ is arbitrary of characteristic zero, and let $f \in \mathrm{g}^{-\phi}$. Then

$$
\left[\left[f, q_{-\phi}\right], q_{-\phi}\right]=-64 f q_{-2 \phi} .
$$

Proof. It is clearly sufficient to prove the first assertion. But by Lemma 7.3,

$$
\begin{aligned}
{\left[\left[f_{\phi}, q_{-\phi}\right], q_{-\phi}\right] } & =4\left[\left[e_{\phi}, q_{-2 \phi}\right], q_{-\phi}\right] \\
& =4\left[\left[e_{\phi}, q_{-\phi}\right], q_{-2 \phi}\right]=8 f_{\phi}\left[b_{\phi}, q_{-2 \phi}\right],
\end{aligned}
$$

by Lemma 6.4 , and this is just $-64 f_{\phi} q_{-2 \phi}$. Q.E.D.

8. The transfer principles. Here we assume that $g^{2 \phi} \neq 0$, as in $\S 7$. But we take $k$ to be an arbitrary field of characteristic zero.

If we attempt to compute directly the conical vectors in the twisted induced modules $X^{\nu}\left(\nu \in a^{*}\right)$, we are confronted with monumental difficulties (cf. the remark at the end of this section). Trying to avoid these problems, we discovered a metamathematical "transfer principle" (Theorem 8.6) which enables us essentially to transfer certain theorems about conical vectors in modules over one semisimple symmetric Lie algebra to theorems about conical vectors in modules over any other semisimple symmetric Lie algebra. This reduces the problem of computing certain conical vectors to any one special case of semisimple symmetric Lie algebra (in which twice the relevant simple restricted root is a restricted root). The proof of this "transfer principle for conical vectors" is based on another metamathematical result (Theorem 8.4) which states that certain kinds of algebraic identities in $\pi_{-} \phi$ can be transferred from one semisimple symmetric Lie algebra to another. The starting point for the proof of this theorem is the "fundamental commutation relation" of the last section.

Let $P=k[w, x, y, z]$, the polynomial algebra in four indeterminates, and define a $P$-module structure on $\pi_{-\phi}$ by the correspondences

$$
\begin{aligned}
w & \mapsto \text { left multiplication by } q_{-\phi}, \\
x & \mapsto \text { left multiplication by } q_{-2 \phi}, \\
y & \mapsto \text { right multiplication by } q_{-\phi}, \\
z & \mapsto \text { right multiplication by } q_{-2 \phi}
\end{aligned}
$$

This $P$-module structure is well defined because $\left[q_{-\phi}, q_{-2 \phi}\right]=0$ in $\pi_{-\phi}$.

Theorem 8.1. Let $f$ be an arbitrary $B_{\theta}$-nonisotropic element of $g^{-\phi}$, and let $P^{f}$ denote the annibilator of $f$ in $P$ under the above module action. 
Then the ideal $p^{f}$ is generated by $x-z$ and $w^{2}-2 w y+y^{2}+64 x$, that is,

$$
P^{\prime}=P(x-z)+P\left(w^{2}-2 w y+y^{2}+64 x\right) .
$$

Proof. Since $q_{-2 \phi}$ is central in $r_{-\phi}$, it is clear that $x-z \in P^{f}$, and so $P(x-z) \subset P^{\prime}$. The fundamental commutation relation, Theorem 7.4, implies immediately that $w^{2}-2 w y+y^{2}+64 x \in P^{f}$, and hence the ideal generated by this element is contained in $P^{f}$. What we must show now is that these two ideals generate $P^{\prime}$.

Let $a \in P^{f}, a \neq 0$, and regard $P$ as $k[x, y, z][w]$. Since the leading coefficient 1 of $w^{2}-2 w y+y^{2}+64 x$ is a unit in $k[x, y, z]$, the Euclidean algorithm implies the existence of $s, t \in k[x, y, z][w]$, where $t$ is a polynomial of degree at most 1 in $w$, such that

$$
a=s\left(w^{2}-2 w y+y^{2}+64 x\right)+t .
$$

Here $t$ is of the form $u+w v$, where $u, v \in k[x, y, z]$. Since $a \in P^{f}, t \in P^{f}$. Also, there exist polynomials $u^{\prime}, v^{\prime} \in k[y, z]$ such that

$$
u \equiv u^{\prime}(\bmod P(x-z)) \text { and } v \equiv v^{\prime}(\bmod P(x-z)) \text {. }
$$

Hence

$$
t \equiv u^{\prime}+w v^{\prime} \quad(\bmod P(x-z))
$$

and so

$$
a \equiv u^{\prime}+w v^{\prime} \quad\left(\bmod P(x-z)+P\left(w^{2}-2 w y+y^{2}+64 x\right)\right) .
$$

In particular, $u^{\prime}+w v^{\prime} \in P^{\prime}$. Write $u^{\prime}=u^{\prime}(y, z)$ and $v^{\prime}=v^{\prime}(y, z)$. Then by the definition of the meriule action of $P$ on $\pi_{-\phi}$, we have

$$
f u^{\prime}\left(q_{-\phi}, q_{-2 \phi}\right)+q_{-\phi} f v^{\prime}\left(q_{-\phi}, q_{-2 \phi}\right)=0,
$$

and so

$$
f\left(u^{\prime}\left(q_{-\phi}, q_{-2 \phi}\right)+q_{-\phi} \nu^{\prime}\left(q_{-\phi}, q_{-2 \phi}\right)\right)-\left[f, q_{-\phi}\right] \nu^{\prime}\left(q_{-\phi}, q_{-2 \phi}\right)=0 .
$$

Set $\alpha(y, z)=u^{\prime}(y, z)+y v^{\prime}(y, z)$ and $\beta(y, z)=-v^{\prime}(y, z)(\alpha, \beta \in k[y, z])$. Then

$$
f a\left(q_{-\phi}, q_{-2 \phi}\right)+\left[f, q_{-\phi}\right] \beta\left(q_{-\phi}, q_{-2 \phi}\right)=0 .
$$

It is sufficient to show that $a=\beta=0$, since then we will have $u^{\prime}=v^{\prime}=0$, and so $a \in P(x-z)+P\left(w^{2}-2 w y+y^{2}+64 x\right)$.

As in the proof of Theorem 5.1, let $\pi_{0} \subset \pi_{1} \subset \pi_{2} \subset \ldots$ be the usual filtration of $\pi_{-\phi}$, and for each $r \in \mathbf{Z}_{+}$, let $\pi_{r}: \pi_{r} \rightarrow \pi_{r}, \pi_{r-1}$ be the canonical map. (Here $\bar{r}_{-1}=0$.) Also, let $\sigma_{r}: S^{r}\left(n_{-\phi}\right) \rightarrow r_{r} / r_{r-1}^{r}$ be the natural map, 
so that $\sigma_{r}$ is a linear isomorphism by the Poincaré-Birkhoff-Witt theorem.

Write

$$
a(y, z)=\sum_{j=0}^{c} \sum_{i=0}^{j} a_{i j} y^{i} z^{j-i}
$$

and

$$
\beta(y, z)=\sum_{j=0}^{d} \sum_{i=0}^{j} b_{i j} y^{i} z^{j-i}
$$

with $c, d \in \mathbf{Z}_{+}$and $a_{i j}, b_{i j} \in k$. If $a \neq 0$, we may assume that some $a_{i c} \neq 0$ $(i=0, \ldots, c)$, and if $\beta \neq 0$, we may also assume that some $b_{i d} \neq 0$ $(i=0, \ldots, d)$. Also, if $\alpha=0$, take $c=0$ and if $\beta=0$, take $d=0$.

Now we claim that $\left[f, q_{-\phi}\right] \in \Re_{2}$ and $\left[f, q_{-\phi}\right] \notin \Re_{1}$. In fact, it is sufficient to prove this when $k$ is algebraically closed. But then a suitable multiple of $f$ may be taken as the $f_{\phi}$ of $\$ 7$, and the claim follows from Lemma 7.3. In particular, $f \alpha\left(q_{-\phi}, q_{-2 \phi}\right) \in \pi_{2 c+1}$ and $\left[f, q_{-\phi}\right] \beta\left(q_{-\phi}, q_{-2 \phi}\right) \epsilon$ $\pi_{2 d+2}$; recall that the sum of these two terms is zero. Either $2 c+1>2 d+2$ or $2 c+1<2 d+2$. Suppose the first inequality holds. Then

$$
\pi_{2 c+1}\left(f a\left(q_{-\phi}, q_{-2 \phi}\right)\right)=0,
$$

so that

$$
\pi_{2 c+1}\left(f \sum_{i=0}^{c} a_{i c} q_{-\phi}^{i} q_{-2 \phi}^{c-i}\right)=0 .
$$

Let

$$
p_{-\phi}^{\prime}=\frac{2}{(\phi, \phi)} p_{-\phi} \text { and } p_{-2 \phi}^{\prime}=\frac{1}{2(\phi, \phi)} p_{-2 \phi}
$$

and set

$$
s=f \sum_{i=0}^{c} a_{i c}\left(p_{-\phi}^{\prime}\right)^{i}\left(p_{-2 \phi}^{\prime}\right)^{c-i} \in S^{2 c+1}\left(n_{-\phi}\right) .
$$

Then

$$
\begin{aligned}
\sigma_{2 c+1}(s) & =\pi_{2 c+1}(\lambda(s))=\pi_{2 c+1}\left(\lambda(f) \sum_{i=0}^{c} a_{i c} \lambda\left(p_{-\phi}^{\prime}\right)^{i} \lambda\left(p_{-2 \phi}^{\prime}\right)^{c-i}\right) \\
& =\pi_{2 c+1}\left(f \sum_{i=0}^{c} a_{i c} q_{-\phi}^{i} q_{-2 \phi}^{c-i}\right)=0 .
\end{aligned}
$$

Hence $s=0$, and so each $a_{i c}=0(i=0, \ldots, c)$. This is only possible if $a=0$. But then $c=0$, and the inequality $2 c+1>2 d+2$ cannot hold. Hence we may assume that $2 c+1<2 d+2$. In this case, 


$$
\pi_{2 d+2}\left(\left[f, q_{-\phi}\right] \beta\left(q_{-\phi}, q_{-2 \phi}\right)\right)=0,
$$

and so

$$
\pi_{2 d+2}\left(\left[f, q_{-\phi}\right] \sum_{i=0}^{d} b_{i d} q_{-\phi}^{i} q_{-2 \phi}^{d-i}\right)=0 .
$$

Since $\left[f, q_{-\phi}\right] \notin r_{1}$ (see above), there exists a nonzero element $g \in S^{2}\left(n_{-\phi}\right)$ such that $\lambda(g) \equiv\left[f, q_{-\phi}\right] \quad\left(\bmod \Re_{1}\right)$. Set

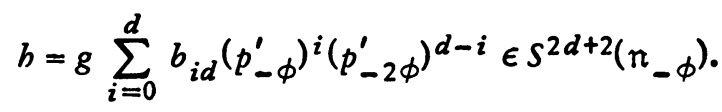

Then

$$
\begin{aligned}
\sigma_{2 d+2}(h) & =\pi_{2 d+2}(\lambda(h))=\pi_{2 d+2}\left(\lambda(g) \sum_{i=0}^{d} b_{i d} \lambda\left(p_{-\phi}^{\prime}\right)^{i} \lambda\left(p_{-2 \phi}^{\prime}\right)^{d-i}\right) \\
& =\pi_{2 d+2}\left(\left[f, q_{-\phi}\right] \sum_{i=0}^{d} b_{i d} q_{-\phi}^{i} q_{-2 \phi}^{d-i}\right)=0 .
\end{aligned}
$$

Hence $h=0$. But $g \neq 0$, so that each $b_{i d}=0(i=0, \ldots, d)$. This proves that $\beta=0$, and so $d=0$. Since $2 c+1<2 d+2$, we also have $c=0$. Thus $a$ is a scalar, and the equation $f a=0$ shows that $\alpha=0$. We have proved that $\alpha=\beta=0$, and hence the theorem. Q.E.D.

Suppose now that $\operatorname{dim} g^{2 \phi}=1$, and suppose there exists an element $r_{-2 \phi} \in g^{-2 \phi}$ such that $r_{-2 \phi}^{2}=q_{-2 \phi}$ in $r_{-\phi}$. (Such an element exists if $k$ is algebraically closed, but otherwise, it might not exist.) Define a new $P$ module structure on $\pi_{-} \phi$ by the correspondences

$$
\begin{aligned}
& w \mapsto \text { left multiplication by } q_{-\phi}, \\
& x \mapsto \text { left multiplication by } r_{-2 \phi}, \\
& y \mapsto \text { right multiplication by } q_{-\phi}, \\
& z \mapsto \text { right multiplication by } r_{-2 \phi}
\end{aligned}
$$

This $P$-module structure is well defined since $\left[q_{-\phi}, r_{-2 \phi}\right]=0$ in $\pi_{-\phi}$.

Theorem 8.2. Under the above bypotheses, let $f \in g^{-\phi}$ be $B_{\theta}$-nonisotropic, and let $P_{f}$ be the annibilator of $f$ in $P$ under the new module action. Then

$$
P_{f}=P(x-z)+P\left(w^{2}-2 w y+y^{2}+64 x^{2}\right) .
$$

Proof. The first part of the proof of Theorem 8.1 carries over to the present situation and shows that is sufficient to prove the following: Let 
$a(y, z), \beta(y, z) \in k[y, z]$, and suppose

$$
f a\left(q_{-\phi}, r_{-2 \phi}\right)+\left[f, q_{-\phi}\right] \beta\left(q_{-\phi}, r_{-2 \phi}\right)=0 .
$$

Then $a=\beta=0$.

It is clearly sufficient to assume that $k$ is algebraically closed and that $f$ is the element $f_{\phi}$ of $\$ \$ 6$ and 7. But then by Lemma 7.3, $\left[f_{\phi}, q_{-\phi}\right]=$ $2 f_{n}\left[f_{\phi}, f_{n}\right]$ (where $f_{n}$ is as in that lemma; see $\$ 6$ ), since $\operatorname{dim} g^{2 \phi}=1$. By Lemma 7.2, $\left[f_{\phi}, f_{n}\right]^{2}=16 q_{-2 \phi}$ in $\pi_{-\phi}$, and since $\left[f_{\phi}, f_{n}\right] \in g^{-2 \phi}$, we must have $4 r_{-2 \phi}= \pm\left[f_{\phi}, f_{n}\right]$. Changing the sign of $f_{n}$ if necessary, we may assume that $4 r_{-2 \phi}=\left[f_{\phi}, f_{n}\right]$. Setting $\alpha^{\prime}(y, z)=\alpha(y, z)$ and $\beta^{\prime}(y, z)=$ $8 z \beta(y, z)$ in $k[y, z]$, we have

$$
f_{\phi} a^{\prime}\left(q_{-\phi}, r_{-2 \phi}\right)+f_{n} \beta^{\prime}\left(q_{-\phi}, r_{-2 \phi}\right)=0,
$$

and it is sufficient to show that $\alpha^{\prime}=\beta^{\prime}=0$.

Now $\left[e_{\phi}, f_{n}\right] \in \mathfrak{m}$ (where $e_{\phi}$ is as in $\S 6$ ), by Lemma 6.2, and so

$$
\left[e_{\phi}, f_{n}\right] \cdot \alpha^{\prime}\left(q_{-\phi}, r_{-2 \phi}\right)=\left[e_{\phi}, f_{n}\right] \cdot \beta^{\prime}\left(q_{-\phi}, r_{-2 \phi}\right)=0
$$

in $r_{-\phi}$, since $q_{-\phi}, r_{-2 \phi} \in r_{-\phi}^{m}$. Also, [ $\left.\left[e_{\phi}, f_{n}\right], f_{\phi}\right]=-6 f_{n}$ by Lemma 4.15 and $\left[\left[e_{\phi}, f_{n}\right], f_{n}\right]=6 f_{\phi}$ by Lemma 6.3 . Hence the application of $\left[e_{\phi}, f_{n}\right]$ to $(*)$ gives

$$
f_{n} \alpha^{\prime}\left(q_{-\phi}, r_{-2 \phi}\right)-f_{\phi} \beta^{\prime}\left(q_{-\phi}, r_{-2 \phi}\right)=0 \text {. }
$$

Abbreviate $\alpha^{\prime}\left(q_{-\phi}, r_{-2 \phi}\right)$ by $\alpha_{0}$ and $\beta^{\prime}\left(q_{-\phi}, r_{-2 \phi}\right)$ by $\beta_{0}$. Multiplying (2) on the right by $\alpha_{0}$, multiplying (3) on the right by $-\beta_{0}$, and adding the two results, we get $f_{\phi}\left(\alpha_{0}^{2}+\beta_{0}^{2}\right)=0$. Since $\mathcal{G}$ has no zero divisors,

$$
\left(\alpha_{0}+(-1)^{1 / 2} \beta_{0}\right)\left(\alpha_{0}-(-1)^{1 / 2} \beta_{0}\right)=a_{0}^{2}+\beta_{0}^{2}=0,
$$

and so $a_{0}= \pm(-1)^{1 / 2} \beta_{0}$. Thus $(*)$ implies that $\alpha_{0}=\beta_{0}=0$. The fact that $\alpha^{\prime}(y, z)=\beta^{\prime}(y, z)=0$ now follows from Theorem 5.1, Case 3. Q.E.D.

Now assume the original hypotheses of this section, so that $g^{2 \phi} \neq 0$. The following consequence of the last two theorems is immediate:

Corollary 8.3. Let $Q$ be the polynomial algebra in two variables over $k$, and let $a_{i}, b_{i} \in Q\left(i=1, \ldots, r, r \in \mathbf{Z}_{+}\right)$. Let $f$ be a $B_{\theta}$-nonisotropic element of $\mathrm{g}^{-\phi}$. Then

$$
\sum_{i=1}^{r} a_{i}\left(q_{-\phi}, q_{-2 \phi}\right) f b_{i}\left(q_{-\phi}, q_{-2 \phi}\right)=0
$$

in $\pi_{-\phi}$ if and only if 


$$
\sum_{i=1}^{r} a_{i} \otimes b_{i} \in P(x-z)+P\left(w^{2}-2 w y+y^{2}+64 x\right),
$$

where we identify $P$ with $Q \otimes Q$ in the natural way. Suppose in addition that $\operatorname{dim} g^{2 \phi}=1$ and that there exists an element $r_{-2 \phi} \in g^{-2 \phi}$ such that $r_{-2 \phi}^{2}=q_{-2 \phi}$. Then

$$
\sum_{i=1}^{r} a_{i}\left(q_{-\phi}, r_{-2 \phi}\right) f b_{i}\left(q_{-\phi}, r_{-2 \phi}\right)=0
$$

in $\pi_{-\phi}$ if and only if

$$
\sum_{i=1}^{r} a_{i} \otimes b_{i} \in P(x-z)+P\left(w^{2}-2 w y+y^{2}+64 x^{2}\right),
$$

where we again identify $P$ with $Q \otimes Q$.

This corollary proves:

Theorem 8.4. (The transfer principle for $\Re_{-\phi}$ ) Let $Q$ be the polynomial algebra in two variables over $k$, and let $a_{i}, b_{i} \in Q\left(i=1, \ldots, r, r \in \mathbf{Z}_{+}\right)$. Let $(g, \theta)$ be a semisimple symmetric Lie algebra over $k$ with symmetric decomposition $g=€ \oplus p$, a a splitting Cartan subspace of $p, \Sigma \subset a^{*}$ the corre. sponding system of restricted roots, $\phi \in \Sigma$ such that $2 \phi \in \Sigma, \pi_{-\phi}$ the universal enveloping algebra of the Lie subalgebra $n_{-\phi}=g^{-\phi} \oplus g^{-2 \phi}$ of $g, \lambda$ : $S\left(n_{-\phi}\right) \rightarrow r_{-\phi}$ the canonical linear isomorphism, $B$ the Killing form of $g$, $B_{\theta}$ the symmetric bilinear form on $g$ defined by the condition $B_{\theta}(x, y)=$ $-B(x, \theta y)$ for all $x, y \in g, f$ a $B_{\theta}$-nonisotropic vector in $g^{-\phi}, p_{-\phi} \in S^{2}\left(g^{-\phi}\right)$ and $p_{-2 \phi} \in S^{2}\left(g^{-2 \phi}\right)$ the canonical elements defined by $B_{\theta}$, and $q_{-\phi}=$ $2 \lambda\left(p_{-\phi}\right) /(\phi, \phi)$ and $q_{-2 \phi}=\lambda\left(p_{-2 \phi}\right) / 2(\phi, \phi) \in \Re_{-\phi}$. Then the truth or fal. sity of equation (4) in $\pi_{-\phi}$ depends only on $a_{i}$ and $b_{i}(i=1, \ldots, r)$ and not on $\mathrm{g}, \theta, a, \phi$ or $f$. Moreover, suppose in addition that $\operatorname{dim} g^{2 \phi}=1$ and that there exists an element $r_{-2 \phi} \in g^{-2 \phi}$ such that $r_{-2 \phi}^{2}=q_{-2 \phi}$. Then the truth or falsity of equation (5) in $\pi_{-\phi}$ depends only on $a_{i}$ and $b_{i}(i=1$, $\ldots, r)$, and not on $g, \theta, a, \phi, f$ or $r_{-2 \phi}$.

In order to apply this theorem to conical vectors, we need:

Lemma 8.5. Suppose $\phi$ and $2 \phi \in \Sigma_{+}$, and let $V$ be a g-module, $v \in$ $V^{m \oplus n_{\phi}}$ a restricted weight vector with restricted weight $\mu \in a^{*}, e_{0} \in g^{\phi}$ and $i, j \in \mathbf{Z}_{+}$. Then

$$
e_{0} \cdot\left(q_{-2 \phi}^{j} q_{-\phi}^{i} \cdot v\right)=y_{i j} \cdot v,
$$

where $y_{i j} \in \Re_{-\phi}$ is given by the formula 


$$
\begin{aligned}
y_{i j}= & -\frac{1}{4} j\left[\theta e_{0}, q_{-\phi}\right] q_{-2 \phi}^{j-1} q_{-\phi}^{i} \\
& -\sum_{m=1}^{i} 2\left(\left(\mu+\rho_{\phi}\right)\left(h_{\phi}\right)+2-4 m\right) q_{-2 \phi}^{j} q_{-\phi}^{i-m}\left(\theta e_{0}\right) q_{-\phi}^{m-1},
\end{aligned}
$$

where $\rho_{\phi}$ is as in Lemma 6.4. Moreover, suppose in addition that $\operatorname{dim} \mathrm{g}^{2 \phi}$ $=1$ and that there exists an element $r_{-2 \phi} \in g^{-2 \phi}$ such that $r_{-2 \phi}^{2}=q_{-2 \phi}$. Then

$$
r_{-2 \phi} e_{0} \cdot\left(r_{-2 \phi}^{j} q_{-\phi}^{i} \cdot v\right)=y_{i j}^{\prime} \cdot v,
$$

where $y_{i j}^{\prime} \in \Re_{-\phi}$ is given by the formula

$$
\begin{aligned}
y_{i j}^{\prime}= & -\frac{1}{8} j\left[\theta e_{0}, q_{-\phi}\right]_{r}^{j-1}{ }_{-2 \phi}^{i} q_{-\phi}^{i} \\
& -\sum_{m=1}^{i} 2\left(\left(\mu+\rho_{\phi}\right)\left(h_{\phi}\right)+2-4 m\right)_{r}^{j+1} q_{-\phi}^{i-m}\left(\theta e_{0}\right) q_{-\phi}^{m-1} .
\end{aligned}
$$

Proof. We may assume that $k$ is algebraically closed and that $e_{0}=e_{\phi}$, so that $\theta e_{0}=-f_{\phi}$. To prove the first assertion, note that

$$
\begin{aligned}
e_{\phi} \cdot\left(q_{-2 \phi}^{j} q_{-\phi}^{i} \cdot v\right)= & \sum_{l=1}^{j} q_{-2 \phi}^{j-l}\left[e_{\phi}, q_{-2 \phi}\right] q_{-2 \phi}^{l-1} q_{-\phi}^{i} \cdot v \\
& +\sum_{m=1}^{i} q_{-2 \phi}^{j} q_{-\phi}^{i-m}\left[e_{\phi}, q_{-\phi}\right] q_{-\phi}^{m-1} \cdot v .
\end{aligned}
$$

By Lemana 7.3, the first term on the right is $1 / 4 j\left[f_{\phi}, q_{-\phi}\right] q_{-2 \phi}^{j-1} q_{-\phi}^{i} \cdot v$. To handle the second term, use Lemma 6.4. Since $v \in V^{\mathrm{m}}$, Lemma 6.2 shows that the second term is

$$
\sum_{m=1}^{i} 2 q_{-2 \phi}^{j} q_{-\phi}^{i-m}\left(\left(\rho_{\phi}-\phi\right)\left(b_{\phi}\right) f_{\phi}+f_{\phi} h_{\phi}\right) q_{-\phi}^{m-1} \cdot v \text {. }
$$

But it was shown in the proof of Lemma 6.5 that $h_{\phi} q_{-\phi}^{m-1}=q_{-\phi}^{m-1}\left(h_{\phi}-4(m-1)\right)$. Thus the term becomes

$$
\sum_{m=1}^{i} 2\left(\left(\mu+\rho_{\phi}\right)\left(h_{\phi}\right)+2-4 m\right) q_{-2 \phi}^{j} q_{-\phi}^{i-m} f_{\phi} q_{-\phi}^{m-1} \cdot v,
$$

and this proves the first assertion of the lemma.

Now suppose that $\operatorname{dim} g^{2 \phi}=1$ and that $r_{-2 \phi}^{2}=q_{-2 \phi}\left(r_{-2 \phi} \in g^{-2 \phi}\right)$. Then 


$$
\begin{aligned}
r_{-2 \phi} e_{\phi} \cdot\left(r_{-2 \phi}^{j} q_{-\phi}^{i} \cdot v\right)= & \sum_{l=1}^{j} r_{-2 \phi}^{j-l+1}\left[e_{\phi}, r_{-2 \phi}\right] r_{-2 \phi}^{l-1} q_{-\phi}^{i} \cdot v \\
& +\sum_{m=1}^{i} r_{-2 \phi}^{j+1} q_{-\phi}^{i-m}\left[e_{\phi}, q_{-\phi}\right] q_{-\phi}^{m-1} \cdot v .
\end{aligned}
$$

The second term is treated exactly as in the first part of the proof, and all that remains is to show that the first term is $(1 / 8) j\left[f_{\phi}, q_{-\phi}\right] r_{-2 \phi}^{j-1} q_{-\phi}^{i} \cdot v$. But $\left[f_{\phi}, q_{-\phi}\right]=2 f_{n}\left[f_{\phi}, f_{n}\right]$ and $\left[f_{\phi}, f_{n}\right]= \pm 4 r_{-2 \phi}$ as in the proof of Theorem 8.2, and so

$$
\left[f_{\phi}, q_{-\phi}\right]= \pm 8 f_{n}^{r}-2 \phi
$$

and

$$
\left[e_{\phi}, r_{-2 \phi}\right]= \pm \frac{1}{4}\left[e_{\phi},\left[f_{\phi}, f_{n}\right]\right]= \pm f_{n},
$$

by Lemma 4.15. Thus the two indicated terms are equal, and the lemma is proved. Q.E.D.

We can now prove:

Theorem 8.6. (The transfer principle for conical vectors.) Let $Q$ be the

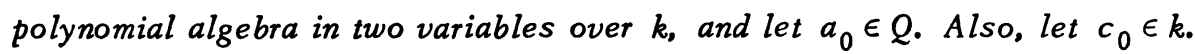
In continuation of the notation of Theorem 8.4 , let $\Sigma_{+}$be a positive system in $\Sigma, \alpha \in \Sigma_{+}$a simple restricted root such that $2 \alpha \in \Sigma, h_{\alpha} \in a$ as defined in $\S 2, \nu \in a^{*}$ such that $\nu\left(h_{a}\right)=c_{0}, X^{\nu}$ the twisted induced g-module (see $\$ 2$ ) and $x_{0} \in X^{\nu}$ the canonical generator. Then the truth or falsity of the assertion " $a_{0}\left(q_{-a}, q_{-2 a}\right) \cdot x_{0}$ is a conical vector in $X$ " depends only on $a_{0}$ and $c_{0}$, and not on $\mathrm{g}, \theta, a, \Sigma_{+}$, $\alpha$ or $\nu$ (except that $\left.\nu\left(h_{a}\right)=c_{0}\right)$. Moreover, suppose in addition that $\operatorname{dim} g^{2 a}=1$ and that there exists an element $r_{-2 a}$ $\epsilon \mathrm{g}^{-2 a}$ such that $r_{-2 \alpha}^{2}=q_{-2 a}$. Then the truth or falsity of the assertion

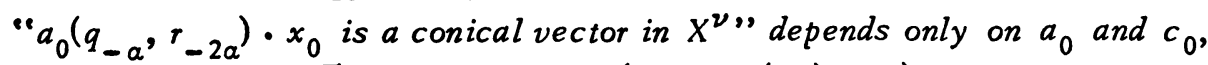
and not on $\mathrm{g}, \theta, a, \Sigma_{+}, a, r_{-2 \alpha}$ or $\nu$ (where $\left.\nu\left(h_{\alpha}\right)=c_{0}\right)$.

Proof. Write $Q=k[x, y]$ and $a_{0}=\Sigma_{i, j=0}^{t} b_{i j} x^{i} y^{j}\left(t \in \mathbf{Z}_{+}\right.$and $\left.b_{i j} \in k\right)$ and assume $a_{0} \neq 0$. In view of Theorem 5.1. (Cases 3 and 4), $a_{0}\left(q_{-a}, q_{-2 a}\right)$. $x_{0}$ is a nonzero m-invariant vector in $X^{\nu}$. Let $e_{0}$ be a $B_{\theta}$-nonisotropic vector in $g^{a}$. Then by Corollary 4.3 and Lemma 6.13 (see the proof of Theorem 6.17), $e_{0} \cdot\left(a_{0}\left(q_{-a}, q_{-2 a}\right) \cdot x_{0}\right)=0$ if and only if $a_{0}\left(q_{-a}, q_{-2 a}\right) \cdot x_{0}$ is conical. But by Lemma 8.5 , this is the case if and only if

$$
\sum_{i, j=0}^{t} b_{i j} y_{i j}=0 \text { in } \pi_{-a}
$$


where $y_{i j} \in \pi_{-a}$ is as in Lemma 8.5, with $\phi$ replaced by $\alpha$ and $\mu$ by $\nu-\rho$ $\left(\rho=1 / 2 \Sigma\left(\operatorname{dim} g^{\psi}\right) \psi, \psi \in \Sigma_{+}\right)$. But $\left(\nu-\rho+\rho_{a}\right)\left(h_{a}\right)=\nu\left(h_{a}\right)=c_{0}$ by Lemma 6.14 , so that

$$
\begin{aligned}
y_{i j}= & -\frac{1}{4} j\left(\theta e_{0}\right) q_{-2 a}^{j-1} q_{-a}^{i+1}+\frac{1}{4} j q_{-\alpha}\left(\theta e_{0}\right) q_{-2 a}^{j-1} q_{-a}^{i} \\
& -\sum_{m=1}^{i} 2\left(c_{0}+2-4 m\right) q_{-2 a}^{j} q_{-a}^{i-m}\left(\theta e_{0}\right) q_{-a}^{m-1}
\end{aligned}
$$

Since $\theta e_{0}$ is a $B_{\theta}$-nonisotropic vector in $g^{-a},(6)$ is an equation of the form treated in Theorem 8.4, with $\phi$ replaced by $a$, and with the $a_{i}$ and $b_{i}$ in Theorem 8.4 dependent only on $a_{0}$ and $c_{0}$. That theorem now implies the first assertion of the present one.

Now assume that $\operatorname{dim} g^{2 a}=1$ and that $r_{-2 a}^{2}=q_{-2 a}\left(r_{-2 a} \in g^{-2 a}\right)$, and let $a_{0} \neq 0$ and $e_{0}$ be as above. By Case 3 of Theorem 5.1, $a_{0}\left(q_{-a}, r_{-2 \alpha}\right) \cdot$ $x_{0}$ is a nonzero m-invariant vector in $X^{\nu}$. Also, since $r_{-2 a}$ is a nonzero element of $\Re^{-}, r_{-2 a} e_{0} \cdot\left(a_{0}\left(q_{-a}, r_{-2 a}\right) \cdot x_{0}\right)=0$ if and only if

$$
e_{0} \cdot\left(a_{0}\left(q_{-a}, r_{-2 a}\right) \cdot x_{0}\right)=0 \text {, }
$$

and this is true if and only if $a_{0}\left(q_{-\alpha}, r_{-2 a}\right) \cdot x_{0}$ is conical, as above. Combining the last parts of Lemma 8.5 and Theorem 8.4 as above, we get the last assertion of the theorem. Q.E.D.

Remark. Of course, the above proof in principle provides an explicit reformulation of the assertion " $a_{0}\left(q_{-\alpha}, q_{-2 \alpha}\right) \cdot x_{0}$ is a conical vector in $X$ " " in terms of $a_{0}$ and $c_{0}$ alone, and similarly for $a_{0}\left(q_{-a}, r_{-2 a}\right) \cdot x_{0}$, under the extra hypotheses. But these reformulations are much too complicated to be useful in determining directly the conical vectors in the induced modules $X^{\nu}$. Instead, we shall compute the conical vectors for a special $g$ (see $\$ 9$ ), and then use Theorem 8.6 to obtain them for general g. The determination of the conical vectors in the special case is not trivial, but at least it can be done.

9. A special case. Following the plan indicated by Theorem 8.6 , we shall determine all the conical vectors in all the twisted induced modules $X^{\nu}$ $\left(\nu \in a^{*}\right)$ for a special semisimple symmetric Lie algebra $(g, \theta)$. Here $(g, \theta)$ will have essentially the same structure as the real semisimple Lie algebra \&u$(2,1)$. Our methods will be special; in fact, one of our main points is that it is too difficult to compute directly the conical vectors in general (cf. $\$ 8$ ). We are grateful to L. Corwin and N. Wallach for their help in carrying out this special case (see the introduction).

Assume $k$ is algebraically closed. Let $g=\mathbb{Q}(3, k)$, the simple Lie al- 
gebra of all traceless $3 \times 3$ matrices over $k$. Let $i=(-1)^{1 / 2}$, and let $k \subset g$ and $p \subset g$ be the spaces of matrices

$$
\left\{\left(\begin{array}{ccc}
a_{11} & a_{12} & a_{13} \\
a_{21} & a_{22} & i a_{21} \\
-a_{13} & -i a_{12} & a_{11}
\end{array}\right)\right\} \text { and }\left\{\left(\begin{array}{ccc}
b_{11} & b_{12} & b_{13} \\
b_{21} & 0 & -i b_{21} \\
b_{13} & i b_{12} & -b_{11}
\end{array}\right)\right\} \text {, }
$$

respectively, where $a_{i j}, b_{i j} \in k$ and $2 a_{11}+a_{22}=0$. Then $g=\mathfrak{E} \oplus \hat{p},[\notin, \mathfrak{E}]$ $\subset E,[E, k] \subset \xi$ and $[k, k] \subset E$, so that the linear automorphism $\theta$ of $g$ which is 1 on $t$ and -1 on $b$ is a Lie algebra automorphism. Thus $(g, \theta)$ is a semisimple symmetric Lie algebra with symmetric decomposition $g=E$ $\oplus$ p.

For all $l, m=1,2,3$, let $E_{l m}$ denote the $3 \times 3$ matrix which is 1 in the $(l, m)$-entry and 0 in all other entries. Let $a$ be the one-dimensional subspace of $h$ spanned by the matrix $h=2\left(E_{11}-E_{33}\right)$. Then $a$ is a splitting Cartan subspace of $p$. Let $a$ be the linear functional on $a$ which is 2 on $h$. Then the set $\Sigma$ of restricted roots of $g$ with respect to $a$ is $\{ \pm a, \pm 2 a\}, g^{0}$ is the set of traceless diagonal matrices, $g^{a}$ is the span of $E_{12}$ and $E_{23}$, $\mathrm{g}^{-a}$ is the span of $E_{21}$ and $E_{32}, 9^{2 a}$ is the span of $E_{13}$, and $\mathrm{g}^{-2 a}$ is the span of $E_{31}$. Also, let $h^{\prime}$ be the matrix $E_{11}-2 E_{22}+E_{33}$. Then the centralizer $m$ of $a$ in $E^{\prime}$ is the span of $h^{\prime}$, and $g^{0}=m \oplus a$.

Let $\Sigma_{+}$be the positive system in $\Sigma$ consisting of $\alpha$ and $2 a$. Then $a$ is the unique simple restricted root. Since $\alpha(b)=2, b=h_{a}$ as defined in $\$ 2$.

The Killing form $B$ of $g$ is given by the formula $B(x, y)=6 \operatorname{tr} x y$. Thus on $g^{-a}$, the form $B_{\theta}(x, y)=-B(x, \theta y)$ is given by the formula

$$
B_{\theta}\left(a E_{21}+b E_{32}, c E_{21}+d E_{32}\right)=-6 i(a d+b c)
$$

$(a, b, c, d \in k)$, and on $g^{-2 a}, B_{\theta}$ is given by

$$
B_{\theta}\left(a E_{31}, b E_{31}\right)=6 a b
$$

$(a, b \in k)$. Hence $\left\{(12)^{-1 / 2}\left(E_{21}+i E_{32}\right),(12)^{-1 / 2}\left(i E_{21}+E_{32}\right)\right\}$ is a $B_{\theta}$-orthonormal basis of $g^{-a}$, and $\left\{6^{-1 / 2} E_{31}\right\}$ is a $B_{\theta}$-orthonormal basis of $g^{-2 a}$. Since the canonical elements $p_{-a} \in S^{2}\left(g^{-a}\right)^{m}$ and $p_{-2 a} \in S^{2}\left(g^{-2 a}\right)^{m}$ (see $\left.\$ 4\right)$ are the sums of the squares of the members of $B_{\theta}$-orthonormal bases of $g^{-a}$ and $g^{-2 a}$, respectively, we have

$$
p_{-a}=(i / 3) E_{21} E_{32} \text { and } p_{-2 a}=(1 / 6) E_{31}^{2} .
$$

The element $x_{\alpha} \in a($ see $\$ 2)$ is $(1 / 12)\left(E_{11}-E_{33}\right)$, so that $(\alpha, \alpha)=$ 
$B\left(x_{a}, x_{a}\right)=1 / 12$. Hence

$$
q_{-\alpha}=24 \lambda\left(p_{-\alpha}\right)=4 i\left(E_{21} E_{32}+E_{32} E_{21}\right)=8 i E_{21} E_{32}+4 i E_{31} \in \pi_{-\alpha}^{m}
$$

and

$$
q_{-2 a}=6 \lambda\left(p_{-2 a}\right)=E_{31}^{2} \in \Re_{-a}^{m},
$$

in the notation of $\$ 5$. We may choose $r_{-2 a}=E_{31} \in g^{-2 a}$ (see Theorem 8.6), since $\operatorname{dim} g^{2 a}=1$ and $E_{31}^{2}=q_{-2 a}$. By Theorem 5.1 (Case 3 ), $\pi_{-a}^{m}$ is the polynomial algebra $k\left[q_{-a}, r_{-2 a}\right]$.

Let $\nu \in a^{*}$. We want to determine the conical vectors in the twisted induced $g$-module $X^{\nu}=V^{\nu-\rho}$ induced from the subalgebra $m \oplus a \oplus n$ of $g$, where $\rho=2 a \in a^{*}$ and $n=g^{a} \oplus g^{2 a}$ (see $\$ 2$ ). Let $x_{0}$ be the canonical generator of $X^{\nu}$. Then

$$
\left(X^{\nu}\right)^{m}=\Re_{-a}^{m} \cdot x_{0}=k\left[q_{-a}, r_{-2 a}\right] \cdot x_{0} .
$$

Thus we must determine the polynomials $a_{0}$ in two variables over $k$ such that $n \cdot\left(a_{0}\left(q_{-a}, r_{-2 a}\right) \cdot x_{0}\right)=0$.

It is hard to guess what conical vectors should look like, but once we know, it is relatively easy to prove that they are in fact conical (in the present special case):

Lemma 9.1. Suppose $\nu\left(h_{a}\right)=2 l, l$ a positive integer, and let

$$
\begin{aligned}
& x=\left(q_{-a}-4 i(l-1) r_{-2 a}\right)\left(q_{-a}-4 i(l-3) r_{-2 a}\right) \cdots \\
& \left(q_{-\alpha}+4 i(l-3) r_{-2 \alpha}\right)\left(q_{-\alpha}+4 i(l-1)_{-2 \alpha}\right) \cdot x_{0}
\end{aligned}
$$

in $X^{\nu}$. Then $x$ is a conical vector.

Proof. Since $E_{13}=\left[E_{12}, E_{23}\right], g^{\alpha}$ generates $n$, and so it is sufficient to show that $E_{12} \cdot x=E_{23} \cdot x=0$. By straightforward computation, using the matrix product relation $E_{\alpha \beta} E_{\gamma \delta}=E_{a \delta}$ if $\beta=\gamma$ and $=0$ if $\beta \neq \gamma(\alpha, \beta, \gamma$, $\delta=1,2,3$ ), we have the following commutation relations in the universal enveloping algebra of $g$ :

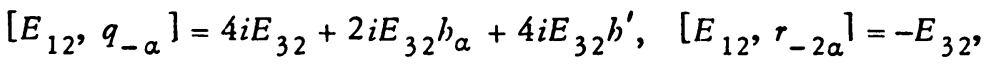

$$
\begin{aligned}
& {\left[E_{23}, q_{-a}\right]=4 i E_{21}+2 i E_{21} h_{\alpha}-4 i E_{21} h^{\prime}, \quad\left[E_{23}, r_{-2 a}\right]=E_{21} \text {. }}
\end{aligned}
$$

Let $a$ be any one of the factors $q_{-a}+4 i j r_{-i a}(j=-(l-1),-(l-3), \cdots$, $l-1)$ appearing in the expression for $x$ in the statement of the lemma. Then $\left[h_{a}, a\right]=-4 a$ and $\left[h^{\prime}, a\right]=0$. Also $h_{a} \cdot x_{0}=(\nu-\rho)\left(h_{\alpha}\right) x_{0}=(2 l-4) x_{0}$ and $h^{\prime} \cdot x_{0}=0$. The above commutation relations thus give 


$$
\begin{aligned}
& E_{12} \cdot x=\left[E_{12},\left(q_{-a}-4 i(l-1) r_{-2 a}\right)\right]\left(q_{-a}-4 i(l-3) r_{-2 a}\right) \\
& \cdots\left(q_{-a}+4 i(l-1) r_{-2 a}\right) \cdot x_{0} \\
& +\left(q_{-a}-4 i(l-1) r_{-2 a}\right)\left[E_{12},\left(q_{-a}-4 i(l-3) r_{-2 a}\right)\right] \\
& \cdots\left(q_{-a}+4 i(l-1) r_{-2 a}\right) \cdot x_{0}+\cdots \\
& =\left(4 i E_{32}+2 i E_{32} h_{a}+4 i E_{32} h^{\prime}+4 i(l-1) E_{32}\right)\left(q_{-a}-4 i(l-3) r_{-2 a}\right) \\
& \cdots\left(q_{-a}+4 i(l-1) r_{-2 a}\right) \cdot x_{0} \\
& +\left(q_{-a}-4 i(l-1) r_{-2 a}\right)\left(4 i E_{32}+2 i E_{32} h_{a}+4 i E_{32} b^{\prime}+4 i(l-3) E_{32}\right) \\
& \cdots\left(q_{-a}+4 i(l-1) r_{-2 a}\right) \cdot x_{0}+\cdots \cdot \\
& =\left(4 i E_{32}+2 i E_{32}(-4(l-1)+2 l-4)+4 i(l-1) E_{32}\right)\left(q_{-a}-4 i(l-3) r_{-2 a}\right) \\
& \cdots\left(q_{-a}+4 i(l-1) r_{-2 a}\right) \cdot x_{0} \\
& +\left(q_{-a}-4 i(l-1) r_{-2 a}\right)\left(4 i E_{32}+2 i E_{32}(-4(l-2)+2 l-4)+4 i(l-3) E_{32}\right) \\
& \cdots\left(q_{-a}+4 i(l-1) r_{-2 a}\right) \cdot x_{0}+\cdots \\
& =0+0+\cdots=0
\end{aligned}
$$

A similar computation shows that $E_{23} \cdot x_{0}=0$. However, $x$ must be written in the "opposite order," as

$$
\begin{aligned}
& \left(q_{-a}+4 i(l-1) r_{-2 a}\right)\left(q_{-a}+4 i(l-3) r_{-2 a}\right) \\
& \cdots\left(q_{-a}-4 i(l-3) r_{-2 a}\right)\left(q_{-a}-4 i(l-1) r_{-2 a}\right) \cdot x_{0},
\end{aligned}
$$

to make the computation exactly parallel to the above one. Q.E.D.

Remark. Because of the flexibility allowed in writing the expression for $\boldsymbol{x}$ in either order in the above proof, we could prove easily that $x$ is conical without appealing to the difficult commutation relations in $\pi$ - This flexibility is lost for Lie algebras $g$ in which the double root space $g^{2 a}$ is more than one-dimensional, since the "square root" $r_{-2 a}$ of $q_{-2 a}$ does not exist.

Now we turn to the uniqueness of the conical vectors.

Lemma 9.2. Let $a_{0}(y, z)$ be a polynomial in two variables over $k$. Then $a_{0}\left(q_{-a}, r_{-2 a}\right) \cdot x_{0}$ is a conical restricted weight vector in $X^{\nu}$ if and only if either $a_{0}$ is a nonzero scalar or else $\nu\left(h_{a}\right)=2 l$, where $l$ is a positive inte. ger, and $a_{0}$ is a nonzero multiple of 


$$
a_{l}=(y-4 i(l-1) z)(y-4 i(l-3) z) \cdots(y+4 i(l-3) z)(y+4 i(l-1) z) .
$$

If $l$ is even, then

$$
a_{l}=\prod_{j=1 ; j \text { odd }}^{l-1}\left(y^{2}+16 j^{2} z^{2}\right)
$$

and if $l$ is odd,

$$
a_{l}=y \prod_{j=2 ; j \text { even }}^{l-1}\left(y^{2}+16 j^{2} z^{2}\right) .
$$

Proof. Let $\mathscr{G}=g^{0}=\mathfrak{m} \oplus a$. Then $\mathscr{G}$ is a Cartan subalgebra of $g$, and the elements $y$ of $\bar{G}$ can be written $y=y_{1} E_{11}+y_{2} E_{22}+y_{3} E_{33}$, where $y_{i} \in k$ and $y_{1}+y_{2}+y_{3}=0$. Define $\lambda_{1}, \lambda_{2}, \lambda_{3} \in \mathfrak{G}^{*}$ by the formulas

$$
\lambda_{1}(y)=y_{1}-y_{2}, \quad \lambda_{2}(y)=y_{2}-y_{3} \text { and } \lambda_{3}(y)=y_{1}-y_{3} .
$$

Then the set $R$ of roots of $g$ with respect to $G$ is $\left\{ \pm \lambda_{1}, \pm \lambda_{2}, \pm \lambda_{3}\right\}$. Denoting the root spaces for $g$ with respect to $G$ by $g^{ \pm \lambda_{i}}$, we have $g^{\lambda_{1}}=k E_{12}$, $g^{\lambda_{2}}=k E_{23}, g^{\lambda_{3}}=k E_{13}, g^{-\lambda_{1}}=k E_{21}, g^{-\lambda_{2}}=k E_{32}$ and $g^{-\lambda_{3}}=k E_{31}$. Let

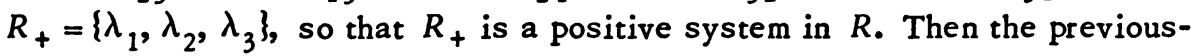
ly defined subalgebra $\mathfrak{m} \oplus a \oplus \mathfrak{n}$ of $g$ is the same as the Borel subalgebra $\mathfrak{b}=\mathfrak{G} \oplus \amalg g^{\lambda}\left(\lambda \in R_{+}\right)$, and $\mathfrak{n}=\amalg g^{\lambda}\left(\lambda \in R_{+}\right)$. Let $\rho^{\prime} \in \mathfrak{G}^{*}$ be the linear functional which is the previously defined $\rho$ on $a$ and 0 on $m$. Then $\rho^{\prime}=$ $1 / 2\left(\lambda_{1}+\lambda_{2}+\lambda_{3}\right)$, i.e., $\rho^{\prime}$ is half the sum of the positive roots of $g$ with respect to $\mathscr{G}$. Also, define $\nu^{\prime} \in \mathfrak{G}^{*}$ by $\nu^{\prime}=\nu$ on $a$ and $\nu^{\prime}=0$ on $\mathrm{m}$. Then the previously defined $t$ wisted induced $g$-module $X^{\nu}$ is the same as the Verma module associated with $\nu^{\prime}$, in the sense of $[2, \$ 7.1 .4]$. That is, $X^{\nu}$ is the $g$-module induced by the character of $\mathfrak{b}$ which is $\nu^{\prime}-\rho^{\prime}$ on $\mathscr{G}$ and 0 on $n$.

In order to describe the Weyl group $W_{R}$ of $g$ with respect to $\bar{G}$, let $\zeta_{1}$ be the space of all (not necessarily traceless) $3 \times 3$ diagonal matrices and let $\mu_{1}, \mu_{2}, \mu_{3} \in E_{1}^{*}$ be the basis of $\xi_{1}^{*}$ dual to the basis $E_{11}, E_{22}, E_{33}$ of $G_{1}$. Now $\hbar^{*}$ may be identified with the space of $k$-linear combinations of $\mu_{1}$, $\mu_{2}$ and $\mu_{3}$, modulo the subspace $k\left(\mu_{1}+\mu_{2}+\mu_{3}\right)$. Then $W_{R}$ is the group of automorphisms of $\xi^{*}$ induced by the six permutations of $\mu_{1}, \mu_{2}$ and $\mu_{3}$. Let $\nu_{1} \in a^{*}$, and define $\nu_{1}^{\prime} \in \mathfrak{F}^{*}$ to be $\nu_{1}$ on $a$ and 0 on $\mathrm{m}$. Then $x_{1}$ $\epsilon X^{\nu}$ is a conical vector with restricted weight $\nu_{1}$ if and only if $x_{1}$ is a (nonzero) $n$-invariant vector $w$ ith weight $\nu_{1}^{\prime}$ for the action of 5 on $X^{\nu}$. But there exists a nonzero $n$-invariant vector in $X^{\nu}$ with weight $\nu_{2} \in G^{*}$ only if there exists $w \in W_{R}$ such that $\nu_{2}+\rho^{\prime}=w \nu^{\prime}$ and $\nu^{\prime}-\left(\nu_{2}+\rho^{\prime}\right)$ is a nonnegative integral linear combination of the elements of $R_{+}$, by [2, Proposition 
7.6.2]. Moreover, the $n$-invariant vectors in $X^{\nu}$ with weight $\nu_{2}$ form at most a one-dimensional space, by a theorem of Verma [2, Théorème 7.6.6]. Let $Z$ be the intersection of the conical space of $X^{\nu}$ with the restricted weight space corresponding to $\nu_{1}$. It follows that if $Z \neq 0$, then $\operatorname{dim} Z=1$, and in this case, either $\nu_{1}=\nu-\rho$, or else $\nu_{1}=-\nu-\rho$ and $\nu=l a$ (i.e., $\nu\left(h_{\alpha}\right)=$ $2 l$ ), where $l$ is a nonnegative integer. Now apply Lemma 9.1. (If $l=0$, then $\nu=0, \nu_{1}=-\rho$ and $Z$ is the span of $x_{0} \circ$ ). Q.E.D.

10. Conclusions. We are now ready to combine the results of $\$ \$ 5,6,8$ and 9 to remove the hypothesis " $2 \alpha \notin \Sigma$ " from Theorems 6.17 and 6.18 .

Let $(g, \theta)$ be a semisimple symmetric Lie algebra over the field $k$ of characteristic zero, $g=\mathfrak{\ell} \oplus$ the symmetric decomposition of $(g, \theta)$, a a splitting Cartan subspace of $p, \Sigma \subset a^{*}$ the corresponding restricted root system, $\Sigma_{+} \subset \Sigma$ a positive system, and $\rho \in a^{*}$ as defined in $\$ 2$. For every $\phi \in \Sigma$, define $h_{\phi}^{\prime} \in a$ to be $h_{\phi}$ if $\operatorname{dim} g^{\phi}>1$ (see $\$ 2$ ) and $2 h_{\phi}$ if $\operatorname{dim} g^{\phi}$ $=1$. Let $s_{\phi}$ be the Weyl reflection with respect to $\phi$ (see $\$ 2$ ). Also, let $q_{\phi}$ and $q_{2 \phi}$ be the elements of the universal enveloping algebra of $g$ defined in $\$ 5$; if $2 \phi \notin \Sigma$, take $q_{2 \phi}=0$.

Here are our main results, which generalize Theorems 6.17 and 6.18:

Theorem 10.1. Let $a \in \Sigma_{+}$be a simple restricted root and $\nu \in a^{*}$. Let $Y$ be the subspace of the twisted induced g-module $X^{\nu}$ spanned by the conical restricted weight vectors with restricted weights of the form $\nu-\rho+c \alpha$ $(c \in k)$; if $\operatorname{dim} a=1$, then $Y$ is the conical space of $X^{\nu}$. Then $\operatorname{dim} Y$ is either $\mathrm{I}$ or 2. If $\nu\left(h_{\alpha}^{\prime}\right)$ is not a positive even integer, then $Y$ is the span of $x_{0}$, the canonical generator of $X^{\nu}$. Suppose $\nu\left(b_{\alpha}^{\prime}\right)=2 l, l$ a positive integer. Then $\operatorname{dim} Y=2$. Define the element $\zeta_{l}$ in the universal enveloping algebra of $g$ as follows: If $\operatorname{dim} g^{\alpha}>1$ and $l$ is even,

$$
\zeta_{l}=\prod_{j=1 ; j \text { odd }}^{l-1}\left(q_{-\alpha}^{2}+16 j^{2} q_{-2 \alpha}\right) ;
$$

if $\operatorname{dim} g^{\alpha}>1$ and $l$ is odd,

$$
\zeta_{l}=q_{-\alpha} \prod_{j=2 ; j \text { even }}^{l-1}\left(q_{-\alpha}^{2}+16 j^{2} q_{-2 \alpha}\right) \text {; }
$$

and if $\operatorname{dim} g^{a}=1, \zeta_{l}=f^{l}$, where $f$ is a nonzero element of $g^{-a}$. Then $Y$ has basis $\left\{x_{0}, \zeta_{l} \cdot x_{0}\right\}$, and $\zeta_{l} \cdot x_{0}$ is a conical restricted weight vector in $X^{\nu}$ with restricted weight $s_{\alpha}^{\nu}-\rho$.

Theorem 10.2. Let a be a simple restricted root, let $\mu, \nu \in a^{*}$, and suppose that $\mu-\nu$ is of the form $c a(c \in k)$. (If $\operatorname{dim} a=1$, then this is automa- 
tic.) Then $\operatorname{Hom}_{\mathrm{g}}\left(X^{\mu}, X^{\nu}\right)$ is at most one-dimensional, and $\operatorname{dim} \operatorname{Hom}_{g}\left(X^{\mu}, X^{\nu}\right)$ $=1$ if and only if either $\mu=\nu$, or else $\mu=s_{\alpha} \nu$ and $\nu\left(h_{\alpha}^{\prime}\right)$ is a nonnegative even integer. Moreover, $\operatorname{dim} \operatorname{Hom}_{g}\left(X^{\mu}, X^{\nu}\right)=1$ if and only if $X^{\mu}$ is isomor. phic to a g-submodule of $X^{\nu}$.

Proof. Theorem 10.2 follows from Theorem 10.1, just as in the proof of Theorem 6.18. To prove Theorem 10.1, note first that the case $2 \alpha \notin \Sigma$ is covered in Theorem 6.17. Suppose that $2 \alpha \in \Sigma$. It is clearly sufficient to assume now that $k$ is algebraically closed. By Lemma $6.16, Y=\left(r_{-a}^{m} \cdot x_{0}\right)^{n}$. Moreover, $\gamma_{-a}^{\mathfrak{m}}$ is the polynomial algebra $k\left[q_{-a}, q_{-2 a}\right]$ if $\operatorname{dim} g^{2 \bar{a}}>1$ and $r_{-a}^{m}$ is the polynomial algebra $k\left[q_{-a}, r_{-2 a}\right]$ if $\operatorname{dim} g^{2 a}=1$, by Theorem 5.1; here $r_{-2 a} \in g^{-2 a}$ and $r_{-2 a}^{2}=q_{-2 a}$ (such an element exists since $k$ is algebraically closed). Hence $Y$ is the set of $m \oplus n$-invariants in $X^{\nu}$ of the form $a_{0}\left(q_{-a}, r_{-2 a}\right) \cdot x_{0}$ if $\operatorname{dim} g^{2 a}=1$ and of the form $a_{0}\left(q_{-a}, q_{-2 a}\right) \cdot x_{0}$ if $\operatorname{dim} g^{2 a}>1$, where $a_{0}$ ranges through the polynomials in two variables over $k$. The stage is set for the application of the transfer principle for conical vectors (Theorem 8.6). Suppose that $\operatorname{dim} 9^{2 a}=1$, and that $a_{0}\left(q_{-a}, r_{-2 a}\right)$ - $x_{0}$ is a conical vector. If $\nu\left(h_{a}\right)$ is not a positive even integer, then $a_{0}$ is a nonzero scalar, by the last part of Theorem 8.6, combined with Lemma 9.2. Suppose now that $\nu\left(h_{\alpha}\right)=2 l$, where $l$ is a positive integer. Then the same two results show that $a_{0}\left(q_{-a}, r_{-2 a}\right) \cdot x_{0}$ is a (nonzero) linear combination of $x_{0}$ and $\zeta_{l} \cdot x_{0}$, in the notation of the theorem. Conversely, $\zeta_{l} \cdot x_{0}$ is, in fact, a conical vector, again by Theorem 8.6 and Lemma 9.2 (or Lemma 9.1). This proves the present theorem in case $\operatorname{dim} g^{2 a}=1$. If $\operatorname{dim} g^{2 a}>1$, the theorem follows from the same argument, this time using the first part of Theorem 8.6. Note that since the polynomials $a_{l}$ in Lemma 9.2 are polynomials in $y$ and $z^{2}$, the space $Y$ has the same description whether $\operatorname{dim} g^{2 a}=1$ or $\operatorname{dim} g^{2 \alpha}>1$. Q.E.D.

Remark. (Cf. the Remark following Theorem 6.17.) In the notation of Theorem 10.1, $\nu\left(h_{\alpha}^{\prime}\right)$ is a nonnegative even integer if and only if $X^{\nu}$ contains an $m$-invariant restricted weight vector with restricted weight $s_{a} \nu-\rho$, or equivalently, a conical restricted weight vector with restricted weight $s_{a} \nu-\rho$. But in general not every $m$-invariant restricted weight vector with restricted weight $s_{\alpha} \nu-\rho$ is conical.

Remark. If $\operatorname{dim} a=1$ and $\operatorname{dim} g^{a}>1$, then $\nu\left(b_{a}^{\prime}\right)=\nu\left(b_{a}\right)$ is a nonnegative even integer if and only if $\nu$ is a nonnegative integral multiple of the unique simple restricted root $a$.

\section{BIBLIOGRAPHY}

1. I. N. Bernštein, I. M. Gel' fand and S. I. Gel'fand, (a) Structure of representa- 
tions generated by highest weight vectors, Funkcional. Anal. i Priložen. 5 (1971), 1-9 = Functional Anal. Appl. 5 ( 1971), 1-8.

(b) Differential operators on the fundamental affine space, Dokl. Akad. Nauk SSSR 195 (1970), 1255-1258. (Russian) MR 43 \#3402.

2. J. Dixmier, Algèbres enveloppantes, Gauthier-Villars, Paris, 1974.

3. M. Duflo, Représentations irréductibles des groupes semi-simples complexes (to appear).

4. Harish-Chandra, Representations of semisimple Lie groups. II, Trans. Amer. Math. Soc. 76 (1954), 26-65. MR 15, 398.

5. S. Helgason, (a) A duality for symmetric spaces with applications to group representations, Advances in Math. 5 (1970), 1-154. MR 41 \#858.

(b) Analysis on Lie groups and homogeneous spaces, CBMS Regional Conference Series in Math., no. 14, Amer. Math. Soc., Providence, R. I., 1972. MR 47 \#5179.

6. B. Kostant, On the existence and irreducibility of certain series of representations, Publ. 1971 Summer School in Math., edited by I. M. Gel'fand, Bolyai-Janós Math. Soc., Budapest (to appear).

7. J. Lepowsky, (a) Algebraic results on representations of semisimple Lie groups, Trans. Amer. Math. Soc. 176 (1973), 1-44.

(b) On the Harish-Chandra homomorphi sm, Trans. Amer. Math. Soc. 208 (1975), 193-218.

(c) Uniqueness of embeddings of certain induced modules (to appear).

(d) On the uniqueness of conical vectors (to appear). appear).

(e) A generalization of $H$. Weyl's "unitary trick", Trans. Amer. Math. Soc. (to

8. G. D. Mostow, Rigidity of locally symmetric spaces, Ann. of Math. Studies, no. 78, Princeton Univ. Press, Princeton, N. J., 1973.

9. C. Rader, Spherical functions on semisimple Lie groups, Thesis and unpublished supplements, University of Washington, 1971.

10. D.-N. Verma, (a) Structure of certain induced representations of complex semisimple Lie algebras, Thesis, Yale University, 1966.

(b) Structure of certain induced representations of complex semisimple Lie algebras, Bull. Amer. Math. Soc. 74 (1968), 160-166; errata, p. 628. MR 36 \#1503; \#5182.

11. N. R. Wallach, Harmonic analysis on homogeneous spaces, Pure and Appl. Math., vol. 19, Dekker, New York, 1973.

12. M. Hu, Determination of the conical distributions for rank one symmetric spaces, Thesis, Massachusetts Institute of Technology, 1973.

DEPARTMENT OF MATHEMATICS, YALE UNIVERSITY, NEW HAVEN, CONNECTICUT 06520 